\title{
Zwischenevaluierung des Programms Innovationsscheck Plus
}

Endbericht 
Diese Studie wurde im Auftrag des Bundesministeriums für Wissenschaft, Forschung und Wirtschaft (BMWFW), Abt. Innovation und Transfer durchgeführt.

VERFASSER/INNEN DES BERICHTS:

Peter Kaufmann (PI)

Laurenz Wolf

Sonja Sheikh

INTERNES REVIEW:

Sonja Sheikh

LAYOUT:

Susanne Fröhlich

Die vorliegende Studie wurde nach allen Maßstäben der Sorgfalt erstellt.

Die KMU Forschung Austria übernimmt jedoch keine Haftung für Schäden oder Folgeschäden, die auf diese Studie oder auf mögliche fehlerhafte Angaben zurückgehen.

Dieses Werk ist urheberrechtlich geschützt. Jede Art von Nachdruck, Vervielfältigung, Verbreitung, Wiedergabe, Übersetzung oder Einspeicherung und Verwendung in Datenverarbeitungssystemen, und sei es auch nur auszugsweise, ist nur mit ausdrücklicher Zustimmung des Auftraggebers der Studie sowie der KMU Forschung Austria gestattet

Mitglied bei:

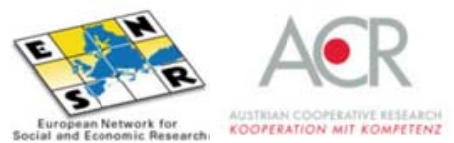




\section{Inhaltsverzeichnis}

1. Zielsetzung und Methoden der Evaluierung .................................... 1

2. Einbettung in das österreichische FTI-Portfolio ............................. 2

3. Innovationsschecks im internationalen Vergleich ........................... 7

4. Das Zielsystem des Innovationsschecks Plus ............................ 20

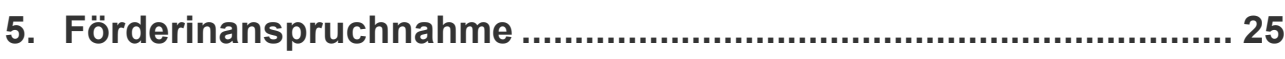

6. Sicht der Unternehmen ................................................................ 36

7. Sicht der Forschungseinheiten ........................................................ 44

8. Schlussfolgerungen und Handlungsempfehlungen .......................46

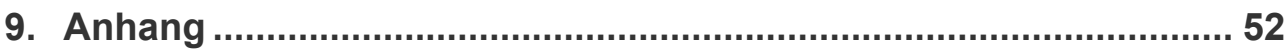

9.1. Weitere Monitoringdaten und Befragungsergebnisse .....................52

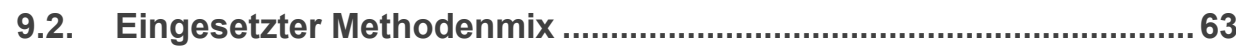

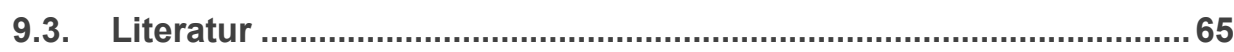

\section{Grafikverzeichnis}

Grafik 1 Positionierung der Innovationsschecks im Portfolio des FFG Bereichs Basisprogramme ................................................. 2

Grafik 2 Das KMU-Paket der FFG....................................................... 5

Grafik 3 Wirkungsmodell Innovationsscheck Plus ………............................ 21

Grafik 4 Verlauf von ISplus Anträgen und Bewilligungsquoten........................26

Grafik 5 Verlauf von IS Anträgen und Bewilligungsquoten ............................26

Grafik 6 Teilnahme von ISplus Forschungseinheiten................................... 28

Grafik $7 \quad$ FFG Vorerfahrung der ISplus Unternehmen .................................... 31

Grafik 8 Entwicklung der FFG Erstfördernehmer im Zeitverlauf ..................... 32

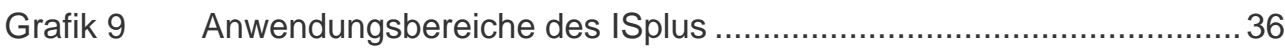

Grafik 10 Heutiger Stand des Projektes und Gesamtprojektkosten...................37

Grafik 11 Weitere Zusammenarbeit mit der Forschungseinrichtung..................40

Grafik 12 Typologie der erreichten ISplus Unternehmen ................................. 41

Grafik 13 Bundesländerverteilung der Unternehmen und FE ..........................52

Grafik 14 Größe der ISplus Unternehmen ....................................................52

Grafik 15 Programmadministration ISplus .....................................................53 
Grafik 16 Erfolgreiche Teilnahme von ISplus Unternehmen in Bereich

Basisprogramme, Jahr der Projekteinreichung .......................... 53

Grafik 17 Anzahl von Erstfördernehmern im Bereich Basisprogramme .......... 56

Grafik 18 Was hätten Sie getan, wenn Sie keinen ISplus bekommen hätten? . 56

Grafik 19 Phasen des Innovationsprozesses...................................... 57

Grafik 20 Phasen des Innovationsprozesses nach Unternehmens-

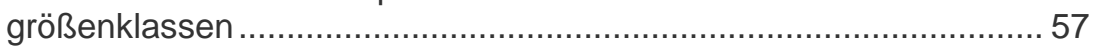

Grafik 21 Übersprungene Phasen seit Projektende .................................. 58

Grafik 22 Heutiger Stand des Projekts nach Unternehmensgrößenklassen ..... 58

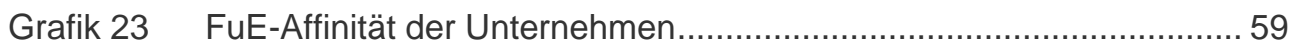

Grafik 24 Vorherige Zusammenarbeit mit einer FE ............................... 59

Grafik 25 Wissenserwerb und Nutzung des neuen Wissen........................6 60

Grafik 26 Zustandekommen der FEI Kooperation .................................... 60

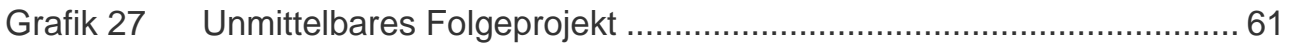

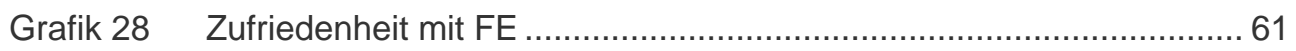

Grafik 29 Weitere Zusammenarbeit mit der Forschungseinheit .................... 62

Grafik 30 Planung von Zusammenarbeit mit Forschungseinheiten ................. 62

\section{Tabellenverzeichnis}

Tabelle 1 Erfolgreiche Projektanträge von Unternehmen mit ISplus Projekten im FFG Portfolio ............................................. 30

Tabelle 2 Einfluss des ISplus auf den Bereich BP ............................... 54

Tabelle 3 Einfluss des ISplus auf das restliche FFG Portfolio ....................... 55

\section{Abkürzungen}

FE Forschungseinheit (AUF, FH, UNI)

AUF Außeruniversitäres Forschungsinstitut

$\mathrm{FH} \quad$ Fachhochschule

UNI Universitätsinstitut

KMU Klein- und Mittelunternehmen (nach EU-Definition)

IS Innovationsscheck (€ 5.000,-)

ISplus Innovationsscheck Plus (€ 10.000,- plus 20\% Selbstbehalt) 


\section{Zielsetzung und Methoden der Evaluierung}

Aufbauend auf den Erfahrungen mit dem Innovationsscheck ( $€$ 5.000,- bzw. IS) hat das damalige Bundesministerium für Wirtschaft, Familie und Jugend in Kooperation mit der Österreichischen Forschungsförderungsgesellschaft (FFG) am 22. Juni 2011 den 'Innovationsscheck Plus' ( $€$ 10.000,- bzW. ISplus) gestartet. Gegenüber dem ,kleinen' Innovationsscheck € 5.000,- sollten mit dem Innovationsscheck Plus gemäß Programmdokument Innovationsvorhaben ,größeren Umfangs und höherer Komplexität' angestoßen werden, damit Unternehmen FuE auch tatsächlich aufnehmen.

Ziel der Zwischenevaluierung war eine Analyse der Konzeption, Umsetzung, des Zielbeitrags und der zum jetzigen Zeitpunkt, drei Jahre nach dessen Einführung, feststellbaren Wirkungen dieses Programms. Darüber hinaus sollten klare Empfehlungen und Maßnahmenvorschläge über die Weiterführung und allfällige Weiterentwicklung des Innovationsschecks Plus abgeleitet werden.

Die Evaluierung wurde im Zeitraum Oktober bis Dezember 2014 (innerhalb von zwei Monaten) durchgeführt und basierte auf einer ausführlichen Literatur- und Dokumentenanalyse (auch auf internationaler Ebene), einer Analyse der Monitoringdaten der FFG zu den Fördernehmern des Innovationsscheck Plus, telefonischen Interviews mit einem repräsentativen Sample von 42 geförderten KMU, einer Fokusgruppe mit ausgewählten VertreterInnen von Wiener Universitäten und Forschungseinrichtungen sowie weiteren 10 Interviews mit Forscherlnnen vorwiegend aus den Bundesländern, bei denen Innovationsschecks eingelöst wurden. Ein abschließender Reflexionsworkshop mit den Auftraggebern sowie VertreterInnen der FFG am 1. Dez. 2014 rundete das Evaluationsdesign ab. Siehe den Anhang für Details zur Umsetzung der einzelnen Methoden.

Die Ergebnisse der Evaluierung werden in diesem Bericht konzise zusammengefasst, wobei die Kapitel 1 bis 3 vor allem auf die Ziele der Evaluation, das Design und Ausgestaltung des Innovationsschecks Plus, auch im internationalen Vergleich eingeht. Kapitel 4 beschreibt die Wirkungslogik des Programms als Grundlage für die darauf folgende Wirkungsanalyse. In Kapitel 5 werden die unmittelbaren Resultate (Outputs) aufgrund der FFG Monitoringdaten aufbereitet, und in Kapitel 6 die bislang feststellbaren Verhaltensänderungen in der Zielgruppe (Outcomes) sowie die erste Wirkungen (Impacts) des Innovationsschecks Plus dargestellt. Kapitel 7 geht auf die Sichtweise der wichtigsten Intermediäre, die Forschungseinheiten, ein. Kapitel 8 beschreibt schließlich die Schlussfolgerungen aus den Analysen sowie die Handlungsempfehlungen. 


\section{Einbettung in das österreichische FTI- Portfolio}

Die Innovationsschecks sind Teil eines Maßnahmenpakets, das seitens des Bundesministeriums für Verkehr, Innovation und Technologie (Innovationsscheck $€ 5.000,--)$ und des Bundesministeriums für Wissenschaft, Forschung und Wirtschaft (Innovationsscheck $€ 5.000$,-- sowie Innovationsscheck Plus $€$ $10.000,--)$ in Kooperation mit der Österreichischen Forschungsförderungsgesellschaft FFG zur Verbesserung des speziellen Angebots an die österreichischen KMU umgesetzt wird. Folgende Grafik verdeutlicht die Positionierung der Innovationschecks im Portfolio des Bereichs Basisprogramme der FFG.

Grafik 1 Positionierung der Innovationsschecks im Portfolio des FFG Bereichs Basisprogramme

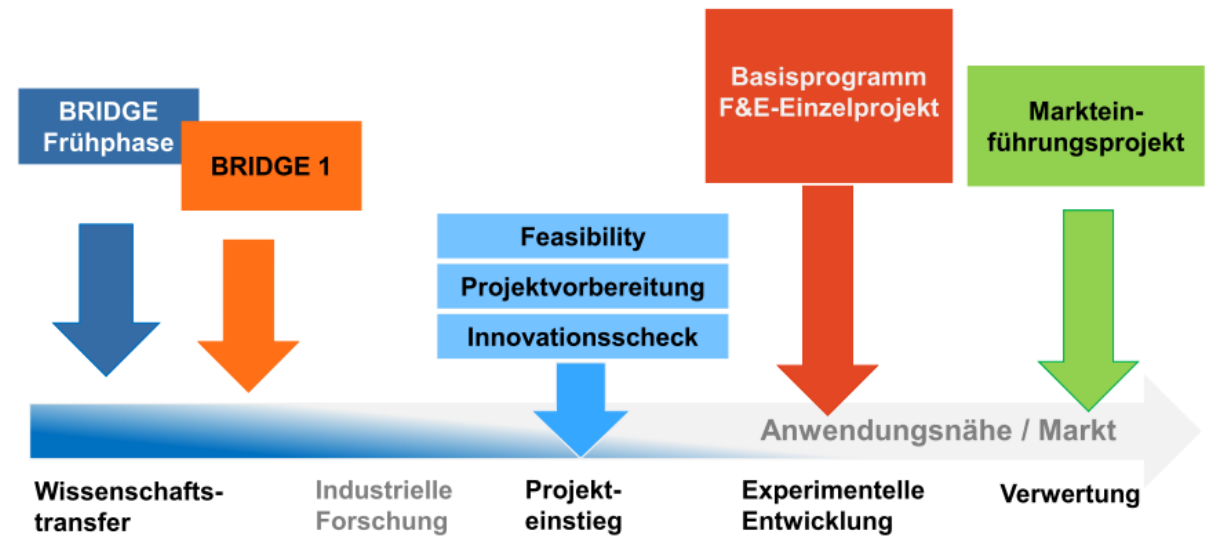

Quelle: Eigendarstellung des Bereichs Basisprogramme der FFG, Präsentation zum „Fahrplan zum innovativen Unternehmen", 25.4.2014

Das Rational der beiden Innovationsscheck-Programme, und damit auch die Mission des Programms ISplus basiert dabei auf der Erkenntnis, dass das bundespolitische Ziel einer FuE-Quote in der Höhe von 3,76\% des BIP bis 2020 (zu zumindest zwei Drittel von der Wirtschaft finanziert) ${ }^{1}$ so erreicht werden soll, dass über die FuE Schwerpunktsetzungen hinaus auch in die Breite der FuEBasis investiert wird. Im letzten Jahrzehnt hat sich die FuE-Quote ausgehenden von unter 2\% im Jahr 2000 durchwegs sehr positiv entwickelt, jedoch zeigt sich in den letzten Jahren eine Stagnation der Entwicklung und wir verharren derzeit (2013) bei $2,88 \% .^{2}$

1 FTI-Strategie der Bundesregierung vom März 2011.

2 BMWFW und BMVIT, Österreichischer Forschungs- und Technologiebericht 2014, S. 15. 
Darüber hinaus zeigen die Resultate des letzten Community Innovation Surveys (Statistik Austria 2014), dass der Anteil der FEl-Ausgaben im Unternehmensbereich im Vergleich zum vorliegenden Betrachtungszeitraum leicht rückläufig war. Davon betroffen waren vor allem KMU. Kleinunternehmen reduzierten die Ausgaben für FEl-Aktivitäten von $1,3 \%$ auf $1,1 \%$ des Umsatzes, Mittelunternehmen von $1,2 \%$ auf $1 \%$ des Umsatzes. Gleichzeitig ging auch der Anteil technologischer Innovatoren im Unternehmensbereich von 44\% auf 39\% zurück, nicht technologische Innovatoren nahmen hingegen zu. Die Betrachtung nach Größenklassen zeigt des Weiteren, dass zwar die Großunternehmen den höchsten Rückgang an innovationsaktiven Unternehmen verzeichneten, allerdings kam es auch im Bereich der Kleinunternehmen zu einer Reduzierung von zwei Prozentpunkten. Die Entwicklung letzterer ist vor allem auf Grund der deutlich höheren Anzahl an Unternehmen problematisch. Im Sinne der antizyklischen Unterstützung von FEl Aktivitäten könnte man daraus eine weitere Begründung von Innovationsförderungen ableiten.

Vor diesem Hintergrund ist das übergeordnete Ziel der beiden Innovationsschecks eine Verbreiterung der Österreichischen Forschungs- und Innovationsbasis durch ein Heranführen von KMU an regelmäßige FuE- bzw. Innovationsleistungen.

Zielgruppe der Programme sind in erster Linie kleinere, bisher nicht regelmäßig innovierende Unternehmen mit Sitz in Österreich, die kein eigenes FuEPersonal haben und daher auf den Wissenstransfer von Forschungseinrichtungen angewiesen sind. Folgende Tabelle gibt einen Überblick über die Ausgestaltung der beiden Innovationsschecks in Österreich. 


\begin{tabular}{|c|c|c|}
\hline Name des Schecks: & Innovationsscheck & Innovationsscheck Plus \\
\hline Förderträger: & \multicolumn{2}{|l|}{ FFG } \\
\hline Verfügbar seit: & seit Nov. 2007 & seit Juli 2011 \\
\hline Zielgruppe: & \multicolumn{2}{|c|}{$\begin{array}{l}\text { KMU in Österreich (ausgeschlossen: Wirtschaftszweig } \\
\text { Landwirtschaft- und Fischereiprodukte) }\end{array}$} \\
\hline $\begin{array}{l}\text { Charakter der Forschungs- } \\
\text { einheit: }\end{array}$ & \multicolumn{2}{|c|}{$\begin{array}{l}\text { außeruniversitäre Forschungseinrichtungen, } \\
\text { Fachhochschulen und Universitäten }\end{array}$} \\
\hline Höhe des Schecks: & $\max . € 5.000,-$ & max. $€ 10.000,-$ \\
\hline Förderungsquote: & $100 \%$ & bis zu $80 \%$ \\
\hline \multirow{3}{*}{ Voraussetzungen: } & \multicolumn{2}{|c|}{$\begin{array}{l}\text { keine Vertragsbeziehung mit der FE im Rahmen eines } \\
\text { FuE-Projektes in den letzten } 5 \text { Jahren } \\
\text { IS kann von einem Unternehmen nur einmal im Kalen- } \\
\text { derjahr bezogen werden }\end{array}$} \\
\hline & $\begin{array}{l}\text { Beantragung eines Folge- } \\
\text { schecks (€ 5.000,-) mit } \\
\text { derselben FE ist einmalig, } \\
\text { in einem darauffolgenden } \\
\text { Kalenderjahr, nach Ab- } \\
\text { schluss des ersten Inno- } \\
\text { vationsschecks } € 5.000,- \\
\text { möglich, wenn dies keine } \\
\text { unmittelbare inhaltliche } \\
\text { Fortsetzung des Vorgän- } \\
\text { gerschecks darstellt. }\end{array}$ & $\begin{array}{l}\text { Beantragung eines Inno- } \\
\text { vationsschecks Plus } \\
\text { ( } 10.000,-) \text { mit derselben } \\
\text { FE ist einmalig, in einem } \\
\text { darauffolgenden Kalender- } \\
\text { jahr, nach Abschluss ei- } \\
\text { nes Innovationsschecks } \\
€ 5.000,- \text { möglich. }\end{array}$ \\
\hline & \multicolumn{2}{|c|}{$\begin{array}{l}\text { Eine Kombination beider Scheckvarianten bzw. ein } \\
\text { Wechsel von einer auf die andere Variante ist nicht } \\
\text { möglich. } \\
\text { Das Risiko einer etwaigen Nichteinlösung der Innovati- } \\
\text { onsschecks liegt bei der FE, da der Scheck im Nach- } \\
\text { hinein und nach Abnahme der Studie durch die FE } \\
\text { ausbezahlt wird. }\end{array}$} \\
\hline Laufzeit in Monaten: & \multicolumn{2}{|l|}{12 Monate } \\
\hline Budget gesamt: & \multicolumn{2}{|l|}{$€ 4.000 .000,-$ (je $€ 2$ Mio.) } \\
\hline Einreichfrist: & \multicolumn{2}{|l|}{ laufend } \\
\hline Kooperationserfordernis: & \multicolumn{2}{|l|}{ ja } \\
\hline $\begin{array}{l}\text { Evaluierungsergebnis, falls } \\
\text { vorhanden: }\end{array}$ & \multicolumn{2}{|c|}{$\begin{array}{l}\text { Durch Technopolis (2011) für den IS; KMU Forschung } \\
\text { Austria (2015) für den ISplus }\end{array}$} \\
\hline Information im Web: & \multicolumn{2}{|c|}{ www.ffg.at/innovationsscheck } \\
\hline
\end{tabular}

Die Zwischenevaluierung des kleinen Innovationsschecks (bis EUR 5.000,-) durch Technopolis (Good und Tiefenthaler 2011) hat dem Programm ein gutes Zeugnis ausgestellt, kam aber zugleich auch zu eindeutigen Empfehlungen zur Optimierung des Programms. Der Bedarf für eine Erhöhung der Obergrenze bzw. eines Zweitprogramms mit höheren Grenzwerten gehörte jedoch nicht dazu. Die Empfehlung für eine Beibehaltung des Ausmaßes des Innovationsschecks wurde argumentiert, indem Vergleiche zu anderen Programmen in 
Europa (UK, DE, IRL, CH, NL - letzteres gestoppt) gemacht wurden - und hier die EUR 5.000,- bereits als ,relativ großzügig' interpretiert wurden. Weiters wurde in den Raum gestellt, dass bei einer Erhöhung über eine strengere Abwicklung und Kontrolle nachgedacht werden müsste, und dies mit einem niedrigschwelligen Instrument wie dem Innovationsscheck nur schwer vereinbar wäre.

Diese Programme sind Teil des KMU-Pakets der FFG zur Unterstützung des Prozesses zum innovativen Unternehmen, das beginnend mit den beiden Innovationsschecks, über Feasibility Studien hin zum Basisprogramm und Markt.Start leitet (siehe Grafik). Hierbei ist zu erwähnen, dass die FFG mit der untenstehenden treppenartigen Darstellung einen potenziell aufbauenden Charakter der Programme unterstellt, der jedoch als konzeptionelles Raster zu verstehen ist, und nur in absoluten Ausnahmefällen ganz durchlaufen wird.

\section{Grafik 2 Das KMU-Paket der FFG}

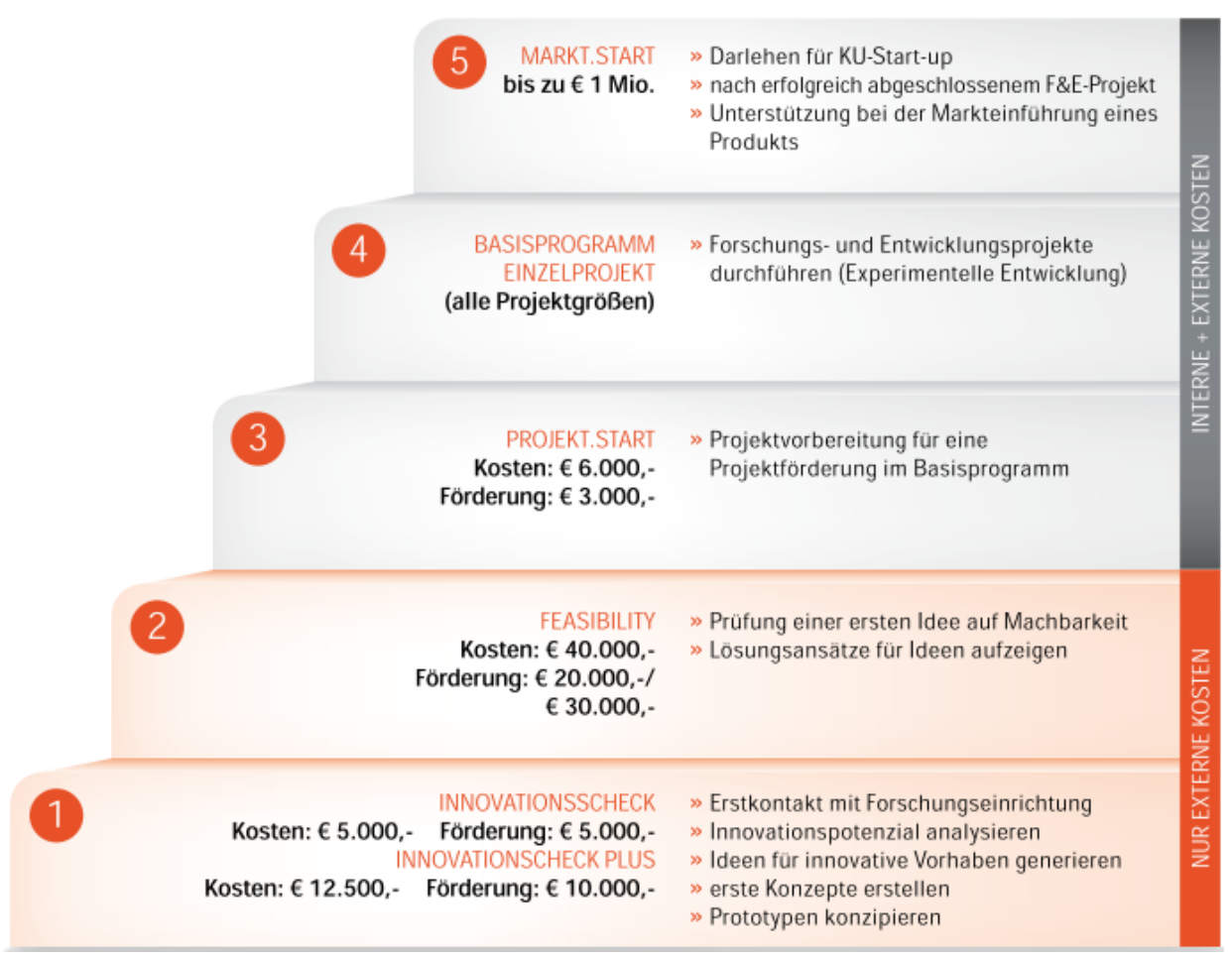

Quelle: FFG

Im Rahmen der gegenständlichen Evaluierung galt es vor diesem Hintergrund zu testen, inwiefern der Innovationsscheck Plus den Bedarf der Unternehmen trifft und als Glied in die oben beschriebene Innovationskette passt. Eine wichtige Frage ist dabei, ob aufgrund des Innovationsschecks Plus mehr Unternehmen (mit wenig FuE Erfahrung) Folgeprojekte durchführen, als dies nur aufgrund des Innovationsschecks passierte. Hier können direkte Vergleiche zwischen den beiden Scheckvarianten vorgenommen werden, da die Evaluationen 
in einem ähnlichen Zeitraum nach dem Beginn des jeweiligen Programms durchgeführt wurden und daher die Prozessdaten vergleichbare Zeiträume abdecken. 


\section{Innovationsschecks im internationalen Vergleich}

Innovationsschecks erfreuen sich aufgrund ihrer Einfachheit einer international großen Beliebtheit. Seit Einführung des ersten Innovationsschecks in den Niederlanden im Jahr 2006 ist die Anzahl der Innovationsscheck Programme in Europa deutlich angestiegen. Im Jahr 2010 gab es etwa 25 unterschiedliche ,innovation voucher systems' allein in den Mitgliedsländern der Europäischen Union, von denen zahlreiche zum heutigen Zeitpunkt noch verfügbar sind. ${ }^{3}$

Die OECD beschreibt Innovation Vouchers als „...small lines of credit provided by governments to small and medium-sized enterprises (SMEs) to purchase services from public knowledge providers with a view to introducing innovations (new products, processes or services) in their business operations". Der Hauptzweck eines Innovationsschecks wird darin gesehen, neue Beziehungen zwischen KMU und Forschungseinrichtungen zu etablieren, die zum einen den direkten Wissenstransfer stimulieren und zum anderen als Katalysator für die Entwicklung von dauerhaften, stabileren Beziehungen fungieren.

Die Implementierung von Innovationsschecks hat im Wesentlichen zwei Intentionen: Zum einen ermöglichen es die Innovationsschecks den KMU, sich mit ihren innovationsbezogenen Problemen an Wissensanbieter zu wenden - etwas, das sie ohne einen solchen Anreiz wahrscheinlich nicht getan hätten. Zum anderen bietet der Scheck den Forschungseinrichtungen, neue Beziehungen zur Industrie aufzubauen, und damit vermehrt die Bedarfe von KMU für ihre zukünftige Tätigkeit kennenzulernen.

Während sich die zugrunde liegende Problemlage und Zielsetzung der Innovationsschecks in den einzelnen Ländern kaum voneinander unterscheiden, variieren die Schecks mitunter deutlich in ihrer Ausgestaltung bzw. Implementierung. In diesem Kapitel geht es vor diesem Hintergrund darum, die Art der Innovationsschecks, die es in ausgewählten Ländern gibt, kurz vorzustellen und auf entsprechende, für Österreich möglicherweise relevante Erfahrungen einzugehen. Insgesamt werden dabei die Innovationsschecks in den folgenden Ländern näher betrachtet: Niederlande, Irland, Deutschland (Sachsen) und Tschechien. Für die Auswahl der Länder war die Verfügbarkeit von Information aufgrund von Programmdokumenten bzw. Programmevaluierungen entscheidend. Weitere Übersichten zu europäischen Innovationsschecks, zu denen es allgemein eher wenig Informationen gibt, sind nur tabellenförmig dargestellt.

${ }^{3}$ http://www.innovationmanagement.se/inside/iframe/single.php?cid=yp/mogritmzep\&p=1724 


\section{Niederlande}

Die Niederlande waren die ersten, die den Innovationsscheck im Jahr 2006 nach einer umfassenden Pilotphase eingeführt haben. Nachdem die Europäische Kommission den niederländischen ,Innovation Voucher' als ein nachahmenswertes Best Practice Beispiel vorgeschlagen hat, wurde er in zahlreichen Ländern eingesetzt. Auch der österreichische Innovationsscheck hatte das niederländische ,Innovation Voucher Programme‘ als Vorbild.

\begin{tabular}{|c|c|c|}
\hline Name des Schecks: & \multicolumn{2}{|l|}{ InnovatieVouchers } \\
\hline Förderträger: & \multicolumn{2}{|l|}{ Agentschap.NL } \\
\hline Verfügbar seit: & \multicolumn{2}{|c|}{$\begin{array}{l}\text { (Pilot: } 2004 \text { und 2005) } 2006 \text { - } 2011 \text { fixes Instrument } \\
\text { (seit } 2010 \text { auch private „innovation vouchers“) }\end{array}$} \\
\hline Zielgruppe: & \multicolumn{2}{|c|}{$\begin{array}{l}\text { Kleine und mittlere Unternehmen (ausgenommen: } \\
\text { Landwirtschaftlicher Sektor) }\end{array}$} \\
\hline $\begin{array}{l}\text { Charakter der Forschungs- } \\
\text { einheit }\end{array}$ & \multicolumn{2}{|c|}{$\begin{array}{l}\text { Als Forschungseinrichtungen konnten bestimmte öffent- } \\
\text { liche und halböffentliche Einrichtungen, die entspre- } \\
\text { chend gelistet waren, beauftragt werden. Diese Liste } \\
\text { umfasste sowohl Universitäten als auch Fachhochschu- } \\
\text { len, führende technische Institute und zwischengeschal- } \\
\text { tete Einrichtungen (z.B. Nederlandse Organisatie voor } \\
\text { Toegepast Natuurwetenschappelijk Onderzoek, TNO) }\end{array}$} \\
\hline Höhe des Schecks: & $\begin{array}{c}\text { Kleiner Innovations- } \\
\text { scheck: } \\
€ 2.500,-\end{array}$ & $\begin{array}{l}\text { Großer Innovationss- } \\
\text { check: } € 7.500,- \text { (zusätzli- } \\
\text { cher Beitrag des KMU: } \\
€ 2.500,-)\end{array}$ \\
\hline Förderungsquote: & $100 \%$ & $75 \%$ \\
\hline Laufzeit in Monaten: & \multicolumn{2}{|l|}{6 Monate } \\
\hline Einreichfrist: & \multicolumn{2}{|l|}{ Bis Ende 2010: jährlich } \\
\hline Kooperationserfordernis: & \multicolumn{2}{|l|}{ ja } \\
\hline Evaluierung vorhanden: & \multicolumn{2}{|c|}{ Ja (Pilotphase 2004 sowie 2004 - 2008) } \\
\hline Information im Web: & \multicolumn{2}{|c|}{$\begin{array}{l}\text { http://www.cpb.nl/en/publication/do-innovation- } \\
\text { vouchers-help-smes-cross-bridge-towards-science }\end{array}$} \\
\hline Zusatzinformation: & \multicolumn{2}{|c|}{$\begin{array}{l}\text { Jedes KMU kann nur um einen Innovationsscheck an- } \\
\text { suchen, es ist jedoch möglich, die Schecks zu bündeln, } \\
\text { d.h. mehrere Unternehmen, die Schecks zugesprochen } \\
\text { bekommen haben, können gemeinsam einen Auftrag an } \\
\text { ein Forschungsinstitut weitergeben. } 10 \text { Innovations- } \\
\text { schecks können gebündelt werden, sie sind jedoch } \\
\text { nicht übertragbar. }\end{array}$} \\
\hline
\end{tabular}


Das Innovation Voucher Programm in den Niederlanden wurde bereits mehrfach evaluiert. Die Evaluierung im Jahr 2006 bezog sich auf die erste Pilotphase des Programms im Jahr 2004, bei der 100 Innovation Vouchers im Lotterieverfahren vergeben wurden (1044 KMU hatten sich für 100 verfügbare Innovationsschecks beworben). ${ }^{4}$ Die Evaluierung basierte auf einem Kontrollgruppenansatz und bescheinigte dem Programm eine hohe Additionalität; 8 von 10 Innovation Vouchers wurden demnach für ein Projekt verwendet, das von den jeweiligen Unternehmen ohne den Innovationsscheck nicht durchgeführt worden wäre. Nach zwei weiteren Pilotversuchen im Jahr 2005, bei denen jeweils 1.000 Innovation Vouchers vergeben wurden, wurden die Innovation Vouchers daraufhin im Jahr 2006 als kontinuierliches Programm des niederländischen Innovationssystems eingeführt. Im Zuge eines Systemwechsels der Förderinstrumentenlogik in den Niederlanden wurde dieses Programm im Jahr 2011 beendet.

Eine weitere Evaluierung des niederländischen Innovation Voucher Programms im Jahr 2008, zeigt, dass es vor allem innovative KMU sind, die an dem Programm teilnehmen, während weniger innovative Unternehmen keinen Gebrauch von den Innovation Vouchers machen. Weitere Analysen auf Basis niederländischer Daten kommen zu dem Schluss, dass KMU tatsächlich vor allem in den frühen Innovationsphasen von externen Netzwerken mit Universitäten profitieren. $^{5}$

Inzwischen wurden jedoch wieder unterschiedlichen Formen von Innovationsschecks in den niederländischen Provinzen gestartet. Es können z.B. Innovationsschecks für ,elektrische Mobilität ${ }^{6}$ sowie im Bereich Landwirtschaft ${ }^{7}$ eingereicht werden.

4 Cornet, M., B. Vroomen and M. van der Steeg (2006). Do Innovation Vouchers Help SMEs to Cross the Bridge towards Science? CBP Discussion Paper No. 58. Netherlands Bureau for Economic Policy Analysis, The Hague

5 Hemert, P., Nijkamp, P., Masurel, E. (2013). From innovation to commercialization through networks and agglomerations: analysis of sources of innovation, innovation capabilities and performance of Dutch SMEs. Annals of Regional Science 50: 425-452

${ }^{6}$ http://www.rvo.nl/subsidies-regelingen/innovatievouchers-elektrische-mobiliteit (31.10.2014)

7 http://www.gelderland.nl/4/actueel/Nieuws/Nieuwsarchief/2014/2014-1e-kwartaal/ProvincieGelderland-stelt-innovatievouchers-beschikbaar.html (31.10.2014) 


\section{Irland}

Irland folgte mit der Einführung des Innovationsschecks dem Vorreiter Niederlande und startete als zweites Land mit dem Programm. 2008 folgte Nordirland, wo Invest Northern Ireland in Zusammenarbeit mit Enterprise Ireland das Programm implementiert hat und damit den Innovationsscheck in allen Teilen Irlands ermöglichte.

\begin{tabular}{|c|c|c|}
\hline Name des Schecks: & $\begin{array}{l}\text { Standard Innovation } \\
\text { Voucher }\end{array}$ & Fast Track Voucher \\
\hline Förderträger & \multicolumn{2}{|l|}{ Enterprise Ireland } \\
\hline Verfügbar seit: & 2007 & 2010 \\
\hline Zielgruppe & \multicolumn{2}{|c|}{$\begin{array}{l}\text { Kleine Unternehmen, die in Irland registriert sind (Aus- } \\
\text { nahme laut Leitlinien für staatliche Beihilfen) }\end{array}$} \\
\hline $\begin{array}{l}\text { Charakter der Forschungs- } \\
\text { einheit }\end{array}$ & \multicolumn{2}{|c|}{$\begin{array}{l}\text { Nur unter www.innovationvouchers.ie gelistete For- } \\
\text { schungseinrichtungen sind einlöseberechtigt }\end{array}$} \\
\hline Höhe des Schecks: & $€ 5.000,-$ (ohne Ust) & $\begin{array}{l}€ 5,000 \text { und das Unter- } \\
\text { nehmen zahlt die restli- } \\
\text { chen } 50 \% \text { der Projektkos- } \\
\text { ten }\end{array}$ \\
\hline Förderungsquote: & $100 \%$ & $50 \%$ \\
\hline Laufzeit in Monaten: & \multicolumn{2}{|l|}{12 Monate } \\
\hline Einreichfrist: & $\begin{array}{l}3 \text { Einreichphasen pro Jahr } \\
\text { (Quartal 1, 2, 3) }\end{array}$ & jederzeit einreichbar \\
\hline Kooperationserfordernis: & \multicolumn{2}{|l|}{ ja } \\
\hline Evaluierung vorhanden: & \multicolumn{2}{|l|}{ nein } \\
\hline Information im Web: & \multicolumn{2}{|c|}{$\begin{array}{l}\text { http://www.enterprise-ireland.com/en/Research- } \\
\text { Innovation/Companies/Collaborate-with-companies- } \\
\text { research-institutes/Innovation-Voucher.shortcut.html }\end{array}$} \\
\hline Zusatzinformation: & \multicolumn{2}{|c|}{$\begin{array}{l}\text { Einschränkung: Unternehmen können maximal } 3 \text { In- } \\
\text { novation Vouchers verwenden, davon muss einer ein } \\
\text { 50:50 Fast Tack Voucher sein. } \\
\text { Fast Track Voucher: Diese Möglichkeit kann von den } \\
\text { Unternehmen genutzt werden, wenn sie keine Berechti- } \\
\text { gung für einen Standard Innovation Voucher mehr ha- } \\
\text { ben (dh bereits zwei oder mehr Standard Innovation } \\
\text { Voucher genutzt haben) bzw. wenn der zeitliche Druck } \\
\text { bereits so groß ist, dass der Einreichzeitplan für Stan- } \\
\text { dard Innovation Voucher nicht geeignet ist. Dieser Vou- } \\
\text { cher kann jederzeit eingereicht werden und die Zu- bzw. } \\
\text { Absage ist innerhalb von zwei Wochen zu erwarten. } \\
\text { Die Einführung der "pooled voucher”, d.h. der Bünde- } \\
\text { lung führte zu größerer Flexibilität für Gruppen von bis } \\
\text { zu } 10 \text { Unternehmen, die sich zusammentun und ge- } \\
\text { meinsam um einen „pooled voucher“ im Wert von bis zu } \\
€ 50.000,- \text { ansuchen. Dies ermöglicht Unternehmen mit } \\
\text { gleichen Forschungsüberlegungen in größere For- } \\
\text { schungsprojekte inkludiert zu werden. }\end{array}$} \\
\hline
\end{tabular}


Der Innovationsscheck wird in Irland allein aufgrund der starken Nachfrage - es gibt keine Evaluierung - als großer Erfolg betrachtet. Seit seiner Einführung im Jahr 2007 bis 2010 haben über 2.200 irische KMU von dem Förderinstrument Gebrauch gemacht. Neben den beiden Varianten „Standard Innovation Voucher" und "Co-funded Fast Track Voucher" hat Enterprise Ireland daher mit 1. September 2014 als Pilotprojekt auch einen sog. „NEW Innovation Voucher Horizon 2020" mit einer vorläufigen Laufzeit von sechs Monaten eingeführt. Dieser Innovationsscheck soll es kleineren Unternehmen in Irland mit weniger als 50 Mitarbeiter/innen ermöglichen, eine Projektvorbereitung für Horizon 2020 mit einem öffentlichen Wissensanbieter durchzuführen und sich gegebenenfalls zu bewerben. Unternehmen, die bereits an Projekten des 7. EU-Rahmenprogramms teilgenommen haben, sind nicht förderungswürdig. Darüber hinaus dürfen Unternehmen während der Pilotphase nur einen „NEW Innovation Voucher - Horizon 2020" beantragen. Nach der Pilotphase ist ein Review des neuen Innovationsschecks vorgesehen. 


\section{Deutschland (Sachsen)}

Die "InnoPrämie" wurde im Juli 2010 als Pilotvorhaben im Freistaat Sachsen eingeführt und zunächst bis zum 31.12.2012 begrenzt. Die folgende Tabelle zeigt einige Eckpunkte der „InnoPrämie“ in Sachsen, die mit dem Innovationsscheck Plus durchaus vergleichbar ist.

\begin{tabular}{|c|c|}
\hline $\begin{array}{l}\text { Name des } \\
\text { Schecks: }\end{array}$ & $\begin{array}{l}\text { Innovationsprämien für kleine und mittlere Unternehmen im } \\
\text { Freistaat Sachsen (InnoPrämie) }\end{array}$ \\
\hline Förderträger & SAB Sächsische Aufbaubank, Förderbank \\
\hline Verfügbar seit: & 2010 \\
\hline Zielgruppe & $\begin{array}{l}\text { Kleine und mittlere Betriebe (KMU) mit Sitz im Freistaat Sachsen } \\
\qquad \begin{array}{cl}0 & \text { der gewerblichen Wirtschaft } \\
0 & \text { Handwerksbetriebe } \\
0 & \text { Ingenieurdienstleister } \\
0 & \text { Existenzgründer } \\
\end{array} \\
\end{array}$ \\
\hline $\begin{array}{l}\text { Charakter der } \\
\text { Forschungsein- } \\
\text { heit }\end{array}$ & $\begin{array}{l}\text { Externe Forschungs- und Entwicklungsdienstleistungen von } \\
\text { - Hochschulen } \\
\text { - } \quad \text { außeruniversitäre Forschungseinrichtungen } \\
\text { - } \quad \text { privatwirtschaftliche Anbieter }\end{array}$ \\
\hline $\begin{array}{l}\text { Höhe des } \\
\text { Schecks: }\end{array}$ & $€ 10.000$ \\
\hline Förderungsquote: & Bis zu $50 \%$ \\
\hline $\begin{array}{l}\text { Laufzeit in Mona- } \\
\text { ten: }\end{array}$ & 6 Monate \\
\hline Einreichfrist: & laufend \\
\hline $\begin{array}{l}\text { Kooperationser- } \\
\text { fordernis }\end{array}$ & ja \\
\hline $\begin{array}{l}\text { Evaluierung vor- } \\
\text { handen: }\end{array}$ & $\mathrm{Ja}(2010$ - 2012) \\
\hline $\begin{array}{l}\text { Information im } \\
\text { Web: }\end{array}$ & $\begin{array}{l}\text { http://amt24.sachsen.de/ZFinder/verfahren.do?action=showdetail\& } \\
\underline{\text { modul=VB\&id=429475!0 }}\end{array}$ \\
\hline $\begin{array}{l}\text { Zusatz- } \\
\text { information: }\end{array}$ & $\begin{array}{l}\text { Der Antragsteller kann pro Kalenderiahr bis zu zwei Innovations- } \\
\text { prämien beantragen. Die Zuwendung beträgt bis zu } 50 \text { Prozent der } \\
\text { zuwendungsfähigen Ausgaben, insgesamt maximal } 20000 \text { EUR } \\
\text { pro Kalenderjahr. } \\
\text { Förderung von externen FuE-Dienstleistungen: } \\
\text { (a) wissenschaftliche Einstiegsarbeiten im Vorfeld der Entwicklung } \\
\text { eines innovativen Produkts, einer Verfahrensinnovation oder einer } \\
\text { innovativen Dienstleistung (Marktforschung, Machbarkeits-, Werk- } \\
\text { stoffstudien, Studien zur Fertigungstechnik) } \\
\text { (b) umsetzungsorientierte FuE-Tätigkeiten in Form von technischer } \\
\text { Unterstützung und Technologietransferdiensten, die überwiegend } \\
\text { beratenden Charakter haben und darauf ausgerichtet sind, innova- } \\
\text { tive Produkte, Verfahren und Dienstleistungen bis zur Markt- bzw. } \\
\text { Fertigungsreife auszugestalten } \\
\text { (Konstruktionsleistungen, Designleistungen, Produkttests zur Quali- } \\
\text { tätssicherung und Umweltverträglichkeit, Laborleistungen, Zertifizie- } \\
\text { rung) } \\
\text { (c) Investitionen in Verbindung mit Dienstleistungen nach (b) }\end{array}$ \\
\hline
\end{tabular}


Im Jahr 2012 wurde die „Innoprämie“ vom IfS Institut für Stadtforschung und Strukturpolitik evaluiert, die zu dem Schluss kamen, dass der Freistaat Sachsen dieses Instrument fortführen sollte, da die Fördermaßnahme auf einen grundlegenden Bedarf seitens der KMU trifft und bezüglich ihrer Konzeption als sinnvolle Ergänzung zu anderen bestehenden Förderprogrammen auf Landes- und Bundesebene zu sehen ist. Der potenzielle Mehrwert des Instruments wird darin gesehen, dass insbesondere auch "Förderneulinge" für FuE-Aktivitäten erreicht werden (fast drei Viertel der Unternehmen, die eine „InnoPrämie“ in Sachsen erhalten haben, sind sog. Neukunden $)^{8}$, v. a. auch junge, kleine Unternehmen (fast ein Viertel der geförderten Unternehmen ist jünger als drei Jahre) und Unternehmen aus Branchen, die nicht zu den Bereichen der Hoch- bzw. Spitzentechnologie zählen und die ein neues Produkt etc. entwickeln. Für diese Unternehmen stellt die Prämie einen einfach handhabbaren Zugang zur FuE Förderung dar, der sich in dieser Form nicht in anderen Programmen bietet.

Neben diesen „Neueinsteigern“ werden gleichzeitig auch Unternehmen erreicht, die (bereits) in anderen FuE-Förderprogrammen erfolgreich Projekte beantragt haben und durch kontinuierliche FuE-Aktivitäten charakterisiert sind. Hier lautet die Interpretation des IfS, dass die Förderung als zusätzliches Programm zu einer Verstetigung bzw. Intensivierung von FuE beiträgt, indem Unternehmen Projektideen in kleinerem Rahmen testen, welche im Anschluss auch zu größeren Vorhaben führen können. Leider ist aus dem Evaluationsbericht nicht nachvollziehbar, aufgrund welcher empirischen Basis die Autoren zu dieser Schlussfolgerung kommen.

Insgesamt empfiehlt das IfS, dass die Fördermaßnahme nach einem Zeitraum von drei bis vier Jahren erneut evaluiert werden sollte, um Langzeiteffekte der ersten Förderprojekte bewerten zu können (u. a. die wirtschaftliche Entwicklung der Unternehmen) sowie gleichzeitig auf mögliche neue Entwicklungen reagieren zu können. Von Interesse sieht das IfS dabei insbesondere die Frage, ob und inwieweit diejenigen Unternehmen, die sich zum jetzigen Zeitpunkt als Neulinge in der FuE-Förderung identifizieren lassen, künftig in anderen Fördermaßnahmen Projekte beantragen bzw. im Unternehmen kontinuierlich FuETätigkeiten durchführen.

\footnotetext{
${ }^{8}$ Als Neukunden definiert wurden hierbei solche Unternehmen, die im Zeitraum 2000 bis 2012 keine weitere Projektförderung in einem der vier wesentlichen Technologieförderprogramme des Freistaats Sachsen erhalten haben.
} 


\section{Tschechien}

In der Region South Moravian in Tschechien wurde das Innovationsscheck Programm im Jahr 2009 als Teil des dritten Updates der regionalen Innovationsstrategie eingeführt. Damals noch als 100\%-ige Förderung; 2010 wurde die Förderquote auf $75 \%$ gesenkt, seitdem müssen Unternehmen einen Eigenanteil von $25 \%$ leisten. Der tschechische Innovationsscheck weist insofern eine Besonderheit auf, als dass seit 2011 Unternehmen aus dem gesamten Europäischen Wirtschaftsraum diesen Innovationsscheck beantragen können. Firmen aus Österreich, Deutschland, der Slowakei und Großbritannien haben bisher von dieser Möglichkeit Gebrauch gemacht. Seit 2012 können sich sogar Unternehmen weltweit um einen Innovationsscheck in Tschechien bewerben. Aufgrund einer Kürzung des Gesamtbudgets wurde jedoch die Höhe des Schecks von bis dahin $€ 6.000$,- auf $€ 4.000$,- reduziert.

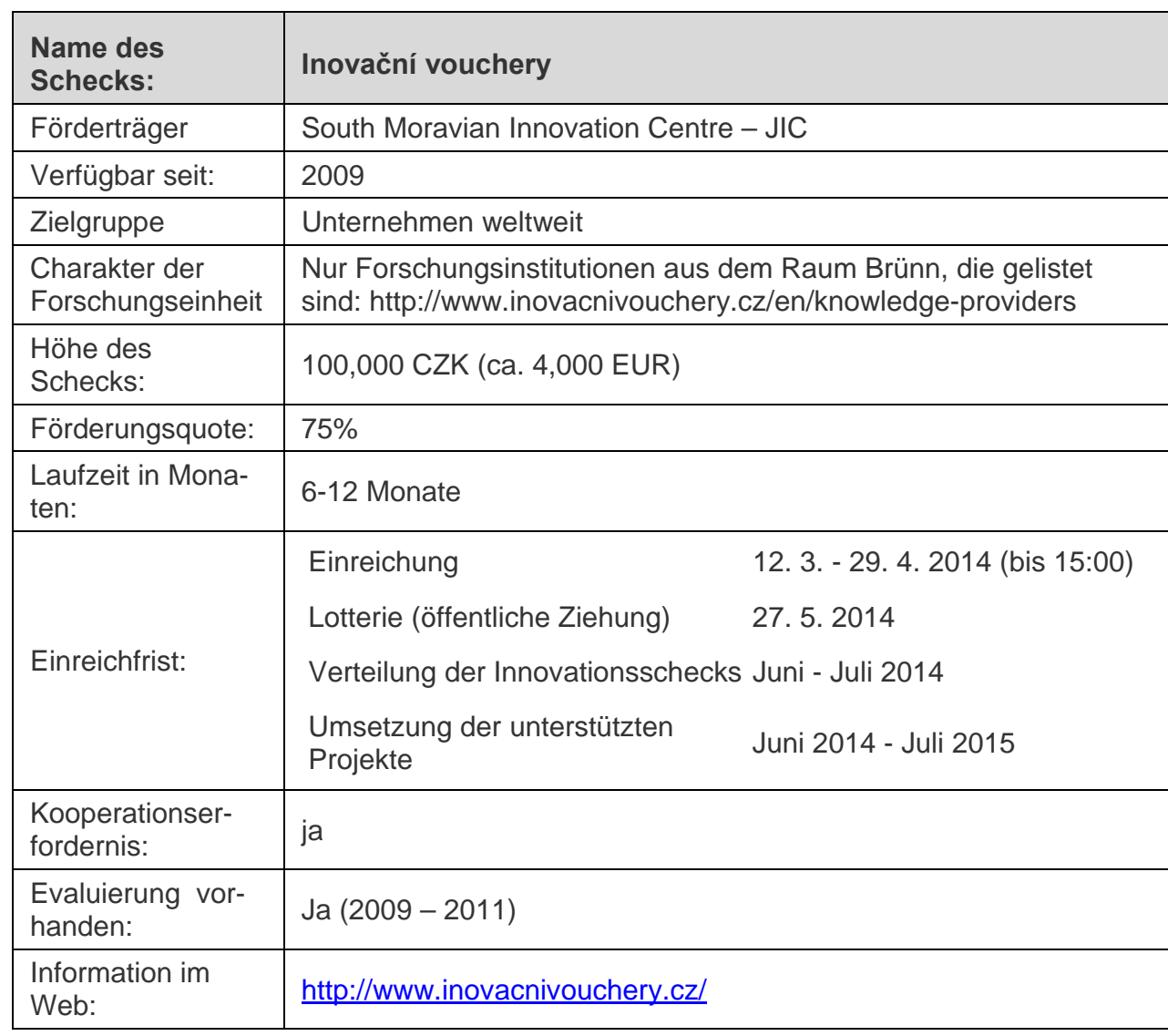

Das tschechische Innovationsscheck Programm wurde im Jahr 2012 evaluiert, wobei der Fokus in erster Linie auf Output Indikatoren lag und im Wesentlichen die Entwicklung der Nachfrage nach dem Innovationsscheck aufgrund der verschiedenen Änderungen nachgezeichnet wurde. So konnte etwa gezeigt werden, dass das Interesse an dem tschechischen Innovationsscheck im Laufe der 
Zeit deutlich angestiegen ist. Dies wird darauf zurückgeführt, dass der Scheck für Unternehmen weltweit geöffnet wurde.

Als wesentlicher Nutzen des Innovationsschecks für die Region South Moravian wird die Bildung von Unternehmensclustern im Umfeld von drei Universitäten in der Region gesehen. Darüber hinaus hat die Evaluierung aufgezeigt, dass sich vor allem Unternehmen mit vorangegangener Kooperationserfahrung mit Forschungseinrichtungen um einen Innovationsscheck bewerben.

\section{Weitere Innovationsschecks:}

\begin{tabular}{|c|c|}
\hline \multicolumn{2}{|r|}{ Großbritannien } \\
\hline Name des Schecks: & Innovation Voucher \\
\hline Förderträger & Innovate UK (former: Technology Strategy Board) \\
\hline Verfügbar seit: & $?$ \\
\hline Zielgruppe & KMU \\
\hline $\begin{array}{l}\text { Charakter der Forschungs- } \\
\text { einheit: }\end{array}$ & $\begin{array}{l}\text { Nicht genauer definiert, Voraussetzung ist, dass es vor } \\
\text { dem Ansuchen noch keine Zusammenarbeit mit der } \\
\text { Forschungseinheit gab. }\end{array}$ \\
\hline Höhe des Schecks: & $£ 5.000,-(\sim>€ 6000)$ \\
\hline Förderungsquote: & $100 \%$ \\
\hline Laufzeit in Monaten: & 6 Monate \\
\hline Einreichfrist: & $\begin{array}{l}\text { Jederzeit, } 100 \text { Innovationsschecks alle drei Monate } \\
\text { (Okt., Jän., April und Juli). }\end{array}$ \\
\hline Kooperationserfordernis: & $\begin{array}{l}\text { Ja, Bedingung: erstmalige Zusammenarbeit mit der } \\
\text { Forschungseinheit. }\end{array}$ \\
\hline $\begin{array}{l}\text { Evaluierungsergebnis, falls } \\
\text { vorhanden: }\end{array}$ & Nein, oder nicht öffentlich zugänglich \\
\hline Information im Web: & $\begin{array}{l}\text { https://vouchers.innovateuk.org/ } \\
\text { https://www.gov.uk/government/news/innovation- } \\
\text { vouchers-for-all }\end{array}$ \\
\hline Zusatzinformation & $\begin{array}{l}\text { Es besteht zusätzlich die Möglichkeit, um lokale oder } \\
\text { regionale Innovationsschecks anzusuchen. Ein Beispiel } \\
\text { wäre das Programm der Universities South West (USW) } \\
\text { im Südwesten (ausgenommen Cornwall). Dieses Pro- } \\
\text { gramm bietet Förderungen für Innovationsprojekte, bei } \\
\text { denen die Expertise einer Universität, einer Fachhoch- } \\
\text { schule oder einer öffentlichen Forschungseinrichtung } \\
\text { gesucht wird. Innovate UK und USW sind übereinge- } \\
\text { kommen, dass Unternehmen, die bei beiden Program- } \\
\text { men als förderbar eingestuft wurden, beide Möglichkei- } \\
\text { ten kombinieren können, um größere Wirksamkeit zu } \\
\text { erzielen. Das USW Programm wird vom European Re- } \\
\text { gional Development Fund unterstützt } \\
\text { (www.usw.ac.uk/business). }\end{array}$ \\
\hline
\end{tabular}




\begin{tabular}{|c|c|}
\hline \multicolumn{2}{|r|}{ Nordirland } \\
\hline Name des Schecks: & Innovation Voucher \\
\hline Förderträger & Invest Northern Ireland \\
\hline Verfügbar seit: & $?$ \\
\hline Zielgruppe & $\begin{array}{l}\text { KMU (Ausnahme: Unternehmen im Transport- und } \\
\text { Landwirtschaftlichen Sektor laut Leitlinien für staatliche } \\
\text { Beihilfen) }\end{array}$ \\
\hline $\begin{array}{l}\text { Charakter der Forschungs- } \\
\text { einheit }\end{array}$ & $\begin{array}{l}\text { Liste mit } 39 \text { renommierten Universitäten, Hochschulen } \\
\text { oder Forschungseinrichtungen } \\
\text { (http://secure.investni.com/static/library/invest- } \\
\text { ni/documents/innovation-vouchers-knowledge- } \\
\text { providers-and-expertise.pdf) }\end{array}$ \\
\hline Höhe des Schecks: & $\begin{array}{l}£ 4000 \text { oder } € 5000 \text { sofern eine Forschungseinheit in } \\
\text { der Republik Irland gewählt wird }\end{array}$ \\
\hline Förderungsquote: & $\begin{array}{l}\text { 100\% beim ersten Innovation Voucher } \\
\text { Sofern beim zweiten Innovation Voucher die gleiche } \\
\text { Kontaktperson bei der Forschungseinheit gewählt wird, } \\
\text { die bereits beim ersten Mal zur Unterstützung beigezo- } \\
\text { gen wurde, beträgt der zweite Voucher nur noch } 90 \% \\
\text { der Projektkosten. Das Unternehmen muss } 10 \% \text { der } \\
\text { Projektkosten und die gesamte Ust. tragen. } \\
\text { Der dritte Innovation Voucher beträgt } 80 \% \text { der Projekt- } \\
\text { kosten (bis zur Maximalhöhe von } £ 4000 \text { ). Das Unter- } \\
\text { nehmen muss } 20 \% \text { der Projektkosten und die gesamte } \\
\text { Ust. übernehmen. }\end{array}$ \\
\hline Laufzeit in Monaten: & Max. 9 Monate \\
\hline Einreichfrist: & während ausgeschriebener Bewerbungsfristen \\
\hline Kooperationserfordernis: & ja \\
\hline $\begin{array}{l}\text { Evaluierungsergebnis, falls } \\
\text { vorhanden: }\end{array}$ & Nein, oder nicht öffentlich zugänglich \\
\hline Information im Web: & http://www.investni.com/innovation-vouchers.html \\
\hline Zusatzinformation & $\begin{array}{l}\text { Es können maximal drei Innovation Vouchers pro Un- } \\
\text { ternehmen vergeben werden. Die Voucher müssen für } \\
\text { unterschiedliche Projekte verwendet werden. }\end{array}$ \\
\hline
\end{tabular}




\begin{tabular}{|c|c|}
\hline \multicolumn{2}{|r|}{ Schweiz } \\
\hline Name des Schecks: & $\begin{array}{l}\text { Innovationsscheck (laufendes Programm: Innovationss- } \\
\text { check 2013-2016) }\end{array}$ \\
\hline Förderträger & Kommission für Technologie und Innovation $\mathrm{KTI}$ \\
\hline Verfügbar seit: & 2011 \\
\hline Ziel & $\begin{array}{l}\text { Wissenschaftsbasierte Innovationen in Zusammen- } \\
\text { arbeit mit Forschungsstätten der Schweiz durch- } \\
\text { zuführen. }\end{array}$ \\
\hline Zielgruppe & KMU bis max. 250 Vollzeitäquivalente \\
\hline $\begin{array}{l}\text { Charakter der Forschungs- } \\
\text { einheit }\end{array}$ & $\begin{array}{l}\text { Als Wissensanbieter und Kooperationspartner der KMU } \\
\text { kommen alle beitragsberechtigten Forschungsstätten } \\
\text { nach Art. } 19 \text { Abs. } 1 \text { FIFG in Betracht } \\
\text { Die KMU sind frei, sich unter diesen einen geeigneten } \\
\text { Projektpartner für ihr Innovationsscheck-Projekt zu } \\
\text { suchen. }\end{array}$ \\
\hline Höhe des Schecks: & maximal 7‘500 Franken \\
\hline Förderungsquote: & $100 \%$ \\
\hline Laufzeit in Monaten: & 12 Monate \\
\hline Budget gesamt: & 2014: 1 Mio Franken \\
\hline Einreichfrist: & jederzeit \\
\hline Kooperationserfordernis: & $\mathrm{Ja}$ \\
\hline $\begin{array}{l}\text { Evaluierungsergebnis, falls } \\
\text { vorhanden: }\end{array}$ & Nicht öffentlich zugänglich \\
\hline Information im Web: & https://www.kti.admin.ch/innoscheck \\
\hline Zusatzinformation & $\begin{array}{l}\text { KMU (dürfen nicht Teil einer Unternehmensgruppe } \\
\text { sein), dürfen nicht an einem laufenden KTI Projekt be- } \\
\text { teiligt sein. Zudem müssen die KMU im Handelsregister } \\
\text { eingetragen sein. Start-ups, die von der KTI unterstützt } \\
\text { werden und über keinen Handelsregistereintrag verfü- } \\
\text { gen, sind berechtigt ein Gesuch für einen Innovationss- } \\
\text { check einzureichen. Ein Unternehmen kann höchstens } \\
\text { alle zwei Jahre einen Innovationsscheck erhalten. }\end{array}$ \\
\hline
\end{tabular}




\section{Deutschland - Bayern}

\begin{tabular}{|c|c|}
\hline Name des Schecks: & $\begin{array}{l}\text { Innovationsgutscheine für kleine Unternehmen und } \\
\text { Handwerksbetriebe }\end{array}$ \\
\hline Förderträger & Bayern Innovativ GmbH \\
\hline Verfügbar seit: & Juni 2009 \\
\hline Zielgruppe & $\begin{array}{l}\text { Unternehmen mit weniger als } 50 \text { Beschäftigten und } \\
\text { einem Vorjahresumsatz oder einer Vorjahresbilanz- } \\
\text { summe von höchstens } 10 \text { Millionen Euro mit Sitz in } \\
\text { Bayern bzw. Existenzgründerinnen und -gründer (Un- } \\
\text { ternehmen, die in Bayern gründen werden) }\end{array}$ \\
\hline $\begin{array}{l}\text { Charakter der Forschungs- } \\
\text { einheit }\end{array}$ & $\begin{array}{l}\text { Externe Forschungseinheit (auch internationale Anbie- } \\
\text { ter können in Anspruch genommen werden) }\end{array}$ \\
\hline Höhe des Schecks: & $\begin{array}{l}\text { Bei } \mathbf{5 0 \%} \text { Förderung je } \\
\text { Innovationsgutschein } 1 \text { : bis zu } 7.500 € \\
\text { Innovationsgutschein } 2 \text { : bis zu } 15.000 € \\
\text { Bei } 60 \% \text { Förderung je } \\
\text { Innovationsgutschein } 1 \text { : bis zu } 9.000 € \\
\text { Innovationsgutschein } 2 \text { : bis zu } 18.000 €\end{array}$ \\
\hline Förderungsquote: & $\begin{array}{l}50 \% \text { bzw. } 60 \% \text { (in Gebieten, die besonders vom demo- } \\
\text { graphischen Wandel betroffen sind) }\end{array}$ \\
\hline Laufzeit in Monaten: & 12 Monate \\
\hline Budget gesamt: & $\begin{array}{l}2009 \text { - 03/2012: } 1.000 \text { Innovationsgutscheine mit einem } \\
\text { Fördervolumen von über acht Millionen Euro }\end{array}$ \\
\hline Einreichfrist: & laufend \\
\hline Kooperationserfordernis & ja \\
\hline Evaluierung, falls vorhanden & Nein, oder nicht öffentlich zugänglich \\
\hline Information im Web: & http://www.innovationsgutschein-bayern.de/ \\
\hline Zusatzinformation & $\begin{array}{l}\text { Für ein Innovationsvorhaben wird maximal ein Innovati- } \\
\text { onsgutschein gewährt. Unabhängig von etwaigen För- } \\
\text { derungen in der Pilotphase können während der Gel- } \\
\text { tungsdauer der aktuell gültigen Richtlinie maximal drei } \\
\text { Innovationsgutscheine und davon maximal zwei als } \\
\text { Innovationsgutschein } 2 \text { beantragt werden } \\
\text { Unternehmen, die sich zu einem größeren Vorhaben } \\
\text { zusammenschließen, können ihre Innovationsgutschei- } \\
\text { ne bündeln. Es können maximal } 4 \text { Innovationsgutschei- } \\
\text { ne gebündelt werden. Dabei müssen alle beteiligten } \\
\text { Unternehmen in den Innovationsprozess direkt einge- } \\
\text { bunden sein und die Verwertung der Produktinnovation } \\
\text { anstreben. Reine Vermarktungs- oder Vertriebspartner } \\
\text { bzw. Subunternehmerschaften sind nicht förderfähig. } \\
\text { Im Übrigen darf neben dieser Förderung für die Finan- } \\
\text { zierung der im Antrag angeführten FuE-Dienstleistung } \\
\text { keine weitere staatliche Förderung in Anspruch ge- } \\
\text { nommen werden. } \\
\text { Soweit keine Vorsteuerabzugsberechtigung besteht, } \\
\text { kann auch die in Rechnung gestellte Umsatzsteuer als } \\
\text { zuwendungsfähig anerkannt werden. }\end{array}$ \\
\hline
\end{tabular}




\section{Fazit}

Die Innovationsschecks in Europa weisen eine Bandbreite an Schwerpunkten und Förderbedingungen auf. Während die meisten hier analysierten Länder den Innovationsscheck entsprechend der OECD-Definition eingeführt haben, um kleinen und mittleren Unternehmen den Zugang zu Wissen zu erleichtern damit diese vermehrt Innovationen (neue Produkte, Prozesse oder Dienstleitungen) in ihre Geschäftstätigkeit integrieren, ist Österreich das einzige der hier untersuchten Länder, das mit dem Innovationsscheck auch dezidiert auf eine Verbreitung der FuE Basis in den Unternehmen abzielt.

Die Höhe und Art der angebotenen Innovationsschecks ist hingegen sehr heterogen. Die Höhe der Schecks reicht von € 2.500,- in den Niederlanden (wurde allerdings 2011 abgeschafft) bis $€ 18.000$,- in Bayern. Ähnlich verhält es sich mit den Förderquoten. Diese reichen von 50\%, beispielsweise bei dem „Fast Track Voucher" in Irland, 60\% in Bayern und Sachsen, bis zu 100\% bei den kleineren Innovationsschecks, die in nahezu allen Ländern keinen Selbstbehalt von den KMU einfordern. Eine Valorisierung der verschiedenen Scheckvarianten wurde bislang kaum vorgenommen.

Auch was die weitere Ausgestaltung der Schecks betrifft, gibt es einige Spezifika. So können sich beispielsweise KMU weltweit um einen tschechischen Innovationsscheck bewerben, solange sie eine lokale Forschungseinheit involvieren. Einige (Bundes-)Länder lassen die Bündelung mehrerer Schecks zu, so können sich etwa in Bayern bis zu vier und in Irland sogar bis zu 10 Unternehmen zu einem größeren Vorhaben zusammenschließen und inre Innovationsschecks bündeln. Auch die Einschränkungen in der Inanspruchnahme der Innovationsschecks weisen eine große Bandbreite auf. So erweist sich die Schweiz z.B. mit max. einem Innovationsscheck alle zwei Jahre als vergleichsweise restriktiv. In Irland ist hingegen die Beantragung von bis zu drei Innovationsschecks pro KMU gleichzeitig möglich, sofern diese für unterschiedliche Innovationsvorhaben eingesetzt werden.

Leider gibt es zumindest im deutsch- und englischsprachigen Raum kaum (zugängliche) Evaluierungen, die die Erfahrungen mit den jeweiligen Innovationsschecks und ihre entsprechenden Wirkungen erfassen. Am ausführlichsten scheint hier noch die vom Institut für Stadtforschung und Strukturpolitik (IfS) durchgeführte Evaluierung der InnoPrämie in Sachsen zu sein, die sich in ihren Ergebnissen auch in weiten Teilen mit der gegenständlichen Evaluierung des österreichischen Innovationsscheck Plus deckt. 


\section{Das Zielsystem des Innovationsschecks Plus}

Die übergeordnete Mission des Innovationsschecks Plus ist eine Verbreiterung der österreichischen Forschungs- und Innovationsbasis durch ein Heranführen von $\mathrm{KMU}$ an regelmäßige FuE- bzw. Innovationsleistungen.

Davon wurden die folgenden spezifischen Programmziele abgeleitet: ${ }^{9}$

1. Stimulierung des Wissenstransfers zwischen dem KMU und dem Wissenschaftssektor

2. Abbau der Schwellenangst von KMU gegenüber den wissenschaftlichen Forschungseinrichtungen ("KMU wagen einen ersten Versuch")

3. Erhöhung der Kooperationsfähigkeit und -bereitschaft zwischen KMU und wissenschaftlichen Forschungseinrichtungen

4. Schließung der Wissenslücke (Forschungseinrichtungen haben das Wissen; KMU holen es ohne Förderung nicht, nicht schnell bzw. nicht im gewünschten Umfang $a b$ ).

Folgende Grafik verdeutlicht vor diesem Hintergrund die Interventionslogik des Programms Innovationsscheck Plus. Dabei werden die in der Sonderrichtlinie Innovationsscheck Plus genannten beiden Zielsetzungen „Stimulierung des Wissenstransfers zwischen dem KMU und dem Wissenssektor" und „SchlieBung der Wissenslücke" aufgrund ihrer kausalen Zusammengehörigkeit zusammengefasst.

Eine Ergänzung gegenüber dem kleinen Innovationscheck bildet die Zielsetzung: „Mit dem Innovationsscheck Plus werden gegenüber den Fördervorhaben beim Innovationsscheck $€ 5.000$,- Innovationsvorhaben größeren Umfangs und höherer Komplexität angestoßen. “10 Hier wird insbesondere eine Verbreiterung der Österreichischen Forschungs- und Innovationsbasis durch Heranführen von $\mathrm{KMU}$ an regelmäßige FuE- bzw. Innovationsleistungen angestrebt. Die starke Betonung der FuE-Komponente im Rahmen des Innovationsschecks Plus stellt, wie bereits dargestellt, ein Spezifikum im internationalen Vergleich dar und ist in diesem Zusammenhang empirisch zu testen.

9 Sonderrichtlinie Innovationsscheck Plus (€ 10.000,- mit Selbstbehalt) des Bundesministeriums für Wirtschaft, Familie und Jugend, Fassung vom 1.1.2014. GZ BMWFJ-98.181/0027-C1/11/2013, S. 3

${ }^{10}$ ebenda 


\section{Grafik 3 Wirkungsmodell Innovationsscheck Plus}

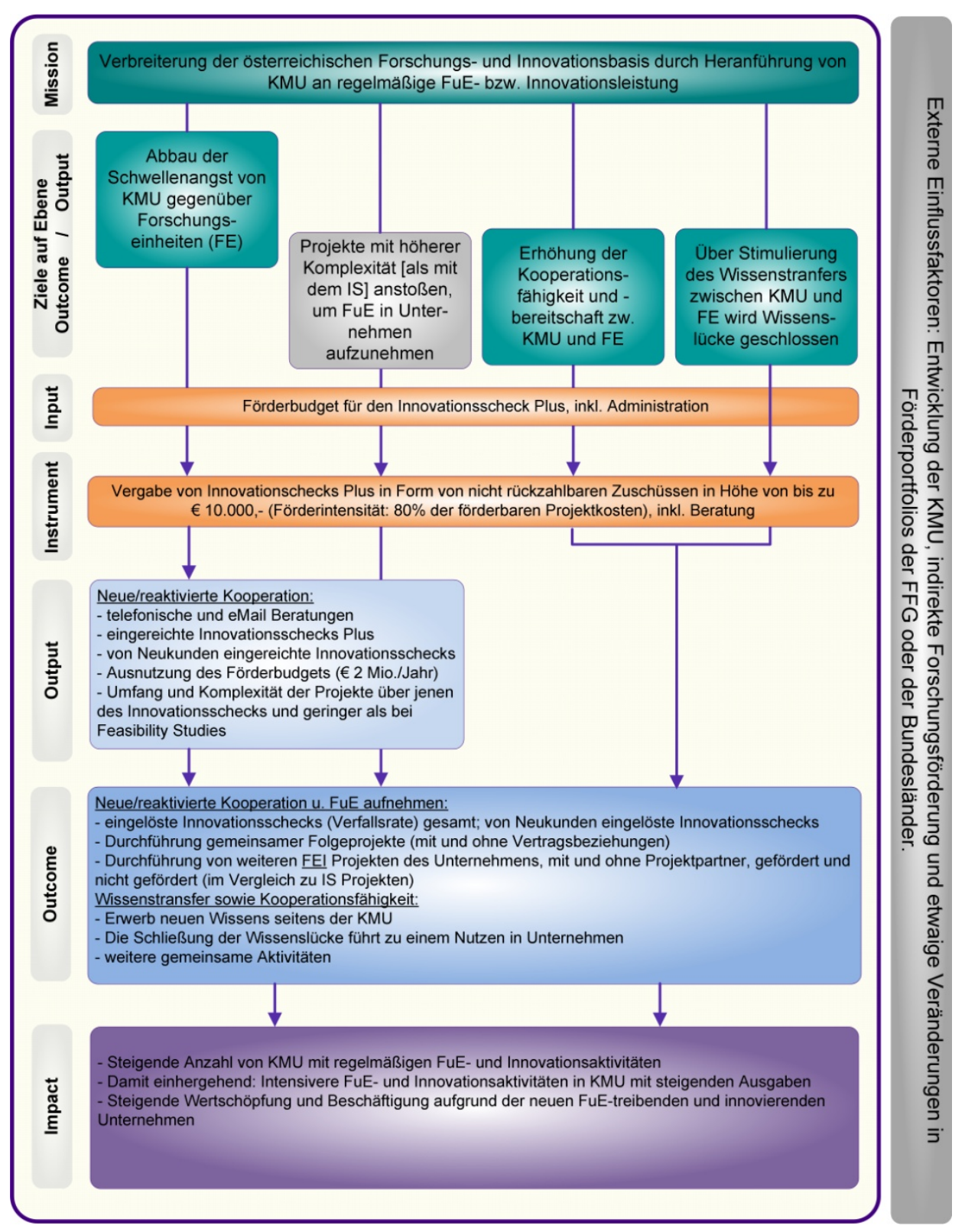

Quelle: KMU Forschung Austria, ausgehend von der Zwischenevaluierung des Innovationsschecks durch technopolis, sowie auf Basis von Programmdokumenten und Interviews

Legende: Output: Unmittelbares Ergebnis der Aktivitäten und Maßnahmen für Kunden oder Stakeholder; Outcome: Mittelbare Effekte auf das Verhalten von Kunden oder Stakeholder (indem Sie etwas mit den Outputs tun und daraus einen Nutzen oder Schaden ziehen); ist auf der Mikroebene angesiedelt und hat tendenziell eine mittelfristige Komponente; Impact: Mittelbare, breitere Effekte auf einen größeren Adressatenkreis bzw. die Gesellschaft, als logische Folge von Outputs über Outcomes bis zu Impacts; Ist auf der Meso- bis Makroebene angesiedelt und hat eine mittel- bis langfristige Komponente. Man unterscheidet oft zwischen Wirkungsebene I (meso) und II (makro). 
Eine Analyse der externen Einflussfaktoren konnte aufgrund von Budgetrestriktionen für diese Evaluation und der Annahme, dass innerhalb des Betrachtungszeitraums von drei Jahren keine größeren Veränderungen stattfanden, nicht umgesetzt werden.

Es stellt sich die Frage, in wie weit der Innovationsscheck Plus tatsächlich in der Lage ist, einen ,Kickstart' zu vermehrter FuE und Innovation in den geförderten KMU zu veranlassen. Auch bei Unternehmen mit FuE Potenzial ist zu vermuten, dass die Aufnahme von FuE nur über einen bestimmten Zeitraum (und der etwaigen Beanspruchung von mehreren Förderprogrammen) passiert; bei Unternehmen ohne FuE Potenzial ermöglicht der Innovationsscheck zwar das Erbringen von Innovationsleistungen - was durchaus im Sinne des Programms ist - kann jedoch nicht zu einer Verbreiterung der FuE-Basis beitragen, da hierzu insbesondere den Kleinstbetrieben in eher traditionellen Branchen meist die Kapazitäten fehlen. Aber auch Unternehmen ohne FuE-Potenzial können die Ziele der Innovationsschecks unterstützen, und können somit eine wichtige Zielgruppe eines Innovationsschecks darstellen.

Die Zielsetzung „Projekte mit höherer Komplexität anstoßen [als mit dem IS], um FuE in Unternehmen aufzunehmen" erweist sich hingegen dann als sinnvoll, wenn in der Zielgruppe ein FuE Potenzial vorliegt. Es stellt sich demnach die Frage, ob FuE Ziele und reine Innovationsziele getrennt ausgewiesen werden sollten, um der Heterogenität der unternehmerischen Rahmenbedingungen und Potenziale gerecht zu werden. Die Alternative dazu wäre, den ISplus nur entweder für Unternehmen mit FuE Potenzial oder rein für Innovationen in Unternehmen zu reservieren. Dies erscheint jedoch aufgrund der oben andiskutieren Breite der bundespolitischen Zielsetzungen als nicht zielführend.

Gemäß der zukünftigen Ausrichtung des Innovationsschecks wären auch entsprechende Indikatoren für das Monitoring des Programms zu definieren. Hierbei werden die folgenden Indikatoren als hilfreich angesehen:

- Verlauf der Nachfrage nach dem Innovationsscheck Plus

o Erreichung der Zielgruppe; Anteil von FFG Erstfördernehmern

- Einstieg in die kontinuierliche Innovationstätigkeit

o Folgeprojekte insgesamt, und von Erstfördernehmern (im Unternehmen, und im Rahmen der FFG)

- Entwicklung der internen und externen Innovations- und FuE-Ausgaben des KMU

o Interne und externe Ausgaben für FuE und Innovation (Investition in Forschungspersonal, Maschinen, Ausrüstung und Software; Erwerb von externem Wissen; Weiterbildungsmaßnahmen im Bereich Innovation)

- Weitere Kooperation mit Forschungseinrichtungen (FE) und Innovationsdienstleistern 
o Art der weiteren Beziehung zur FE

o Aufnahme von Kooperationen mit anderen FE

o Beanspruchung von Innovationsdienstleistern

- Effizienz der Programmadministration

o Verfallsrate

Diese Indikatoren beziehen sich auf die unmittelbaren Ziele der Innovationsschecks auf der Ebene der Input- und Verhaltensadditionalität. Während diese teilweise anhand des FFG Monitorings aufbereitet werden können, müssten einige Indikatoren der Verhaltensadditionalität bei den Unternehmen erfragt werden. Die FuE-Erhebung der Statistik Austria wäre eine weitere, mögliche Datenquelle, die aber aufgrund der österreichspezifischen Gesetzeslage dafür nicht zugänglich ist.

\section{Fazit}

In der Folge wird anhand eines Fragen-Antwort Formats auf die konkreten Evaluierungsfragen eingegangen, die aufgrund des behandelten Themas im jeweiligen Kapitel beantwortet werden können.

- Welche Funktion übt der Innovationsscheck Plus im Kontext zu den Zielsetzungen der FTI-Strategie aus?

„Die Leistungsfähigkeit des Innovationssystems zu erhöhen, ist [daher] eine der grundlegenden Aufgaben einer Strategie für Forschung, Technologie und Innovation, die Österreich zu einem Innovation Leader machen will. Dabei ist von einem breiten Innovationsansatz auszugehen, der technologische, forschungsgetriebene und nicht-technologische Innovationen sowohl in der Sachgüterproduktion als auch im Dienstleistungssektor ebenso einschließt wie ökologische und soziale Innovationen oder Innovationen im öffentlichen Bereich. ${ }^{411}$

Damit passt der Innovationsscheck Plus von seiner Intention her eindeutig in den Kontext der Zielsetzungen der österreichischen FTI-Strategie. Er adressiert mit seiner Mission „Verbreitung der österreichischen Forschungs- und Innovationsbasis durch Heranführung von KMU an regelmäßige FuE bzw. Innovationsleistung" sowie mit seinen Zielsetzungen wesentliche Dimensionen der österreichischen FTI-Strategie in den Bereichen "Innovation und Unternehmensforschung" sowie „Zusammenarbeit von Wissenschaft und Wirtschaft". In wie weit diese Zielsetzungen auch tatsächlich erreichbar sind, gilt es im Rahmen dieser Zwischenevaluierung einer ersten Überprüfung zu unterziehen.

${ }^{11}$ bmvit (2011), Strategie der Bundesregierung für Forschung, Technologie und Innovation, Der Weg zum Innovation Leader, Wien. 
- In wie weit ist das Förderinstrumentarium den Programmzielen angemessen?

Die Ziele des Innovationsschecks Plus stellen neben den Bereich Innovation noch vermehrt auf Forschung und Entwicklung ab. Damit weicht der österreichische Innovationsscheck Plus von den Innovationsschecks in anderen europäischen Ländern ab, die vornehmlich auf eine Erhöhung der Innovationsleistungen von KMU fokussieren (siehe Kapitel 1.3). Aufgrund der Tatsache, dass der Innovationsscheck Plus in ein Förderportfolio eingebettet ist und Teil des KMU-Pakets der FFG darstellt, das beginnend mit den beiden Innovationsschecks, über die Feasibility Studien hin zum Basisprogramm und Markt.Start einen quasi linearen Förderprozess suggeriert, ist davon auszugehen, dass mit dem Durchlaufen (eines Teils) der Förderkette tatsächlich ein Beitrag zur Verbreitung der FuE-Basis in Österreich zu erwarten ist. In wie weit der Innovationsscheck Plus jedoch dazu in der Lage ist - wie in den Programmzielen formuliert - einen Kick-start für einen kontinuierlichen FuE-Prozess in den Unternehmen zu initiieren bleibt einer Ex-post Evaluierung zu prüfen; diese Zwischenevaluierung kann per Design nur erste Hinweise liefern. Die Angemessenheit des Förderinstrumentariums in Bezug auf alle anderen Zielsetzungen des Innovationsschecks Plus steht außer Frage. 


\section{Förderinanspruchnahme}

Seit Einführung des Innovationsschecks Plus per 22. Juni 2011 konnten bis Ende Juni 2014 (Beobachtungszeitraum von drei Jahren) 668 Projektpartner (Unternehmen und Forschungseinheiten) insgesamt 334 Forschungsvorhaben umsetzen. ${ }^{12}$ Darüber hinaus wurden It. FFG Monitoringdaten weitere 172 kooperative Forschungsvorhaben genehmigt und tlw. bereits umgesetzt, allerdings fällt deren Projektabschlussdatum in den Zeitraum nach dem 1. Juli 2014 und liegt damit außerhalb des relevanten Evaluationszeitraums.

Die 334 abgeschlossenen Unternehmensbeteiligungen (durch 329 unterschiedliche Unternehmen) verteilen sich dabei wie folgt auf die Projektabschlussjahre: 2012 wurden 89 Projekte durchgeführt bzw. abgeschlossen, 2013 waren es 152 und im 1. Halbjahr 2014 kam es zu 93 Projektabschlüssen. Im Zeitraum von 2011 bis 2013 lässt sich diesen Zahlen folgend, eine steigende Anzahl an Förderanträgen verzeichnen sowie der tatsächlich genehmigten ISplus-Anträge. Hingegen zeigen die ersten sechs Monaten des Jahres 2014 eine leicht rückläufige Nachfrage. Ein Nachfragerückgang lässt sich im selben Zeitraum auch für den kleinen Innovationsscheck feststellen, wobei dieser u.a. aus der Einführung des ISplus resultiert. Anders formuliert substituierte das Angebot des ISplus die Nachfrage des kleinen Innovationschecks. Mit dem anwachsenden Interesse am ISplus ging auch eine deutliche Steigerung der Beratungsintensität (sowohl eingegangene als auch genehmigte Anträge) im selben Zeitraum einher (siehe Grafik 15 im Anhang). Durch die höhere Beratungsintensität konnte die Genehmigungsquote im 1. Halbjahr 2014 auf 91\% angehoben werden. Mit über 95\% weist darüber hinaus auch die tatsächliche Einlösequote der genehmigten Anträge einen im internationalen Vergleich hohen Wert auf. Auf Grund des regen Interesses im Jahr 2012 wurde das Rahmenbudget von $€ 1,5$ Mio. auf $€ 2$ Mio. ausgeweitet.

\footnotetext{
${ }^{12}$ Projektende fällt It. FFG Monitoringdaten in diesen Zeitraum.
} 
Grafik 4 Verlauf von ISplus Anträgen und Bewilligungsquoten

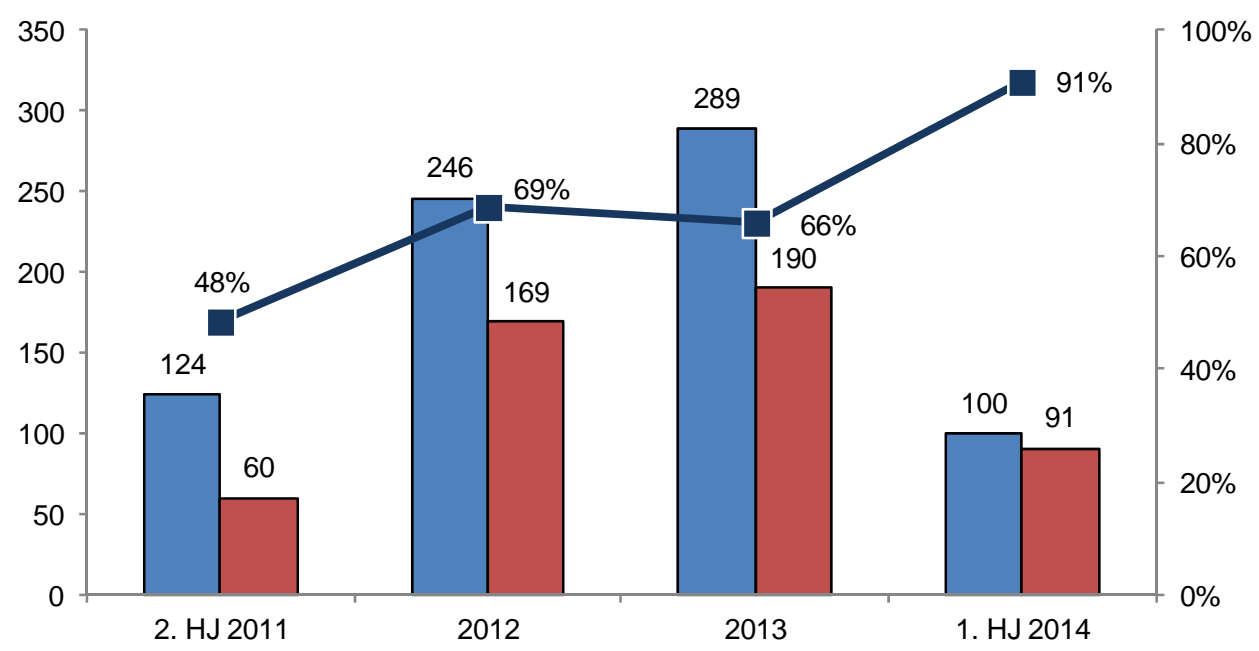

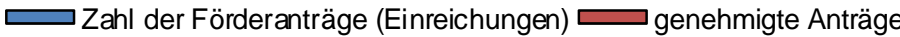

-Genehmigungsquote

Quelle: FFG Monitoring des Bereichs Basisprogramme

\section{Grafik 5 Verlauf von IS Anträgen und Bewilligungsquoten}

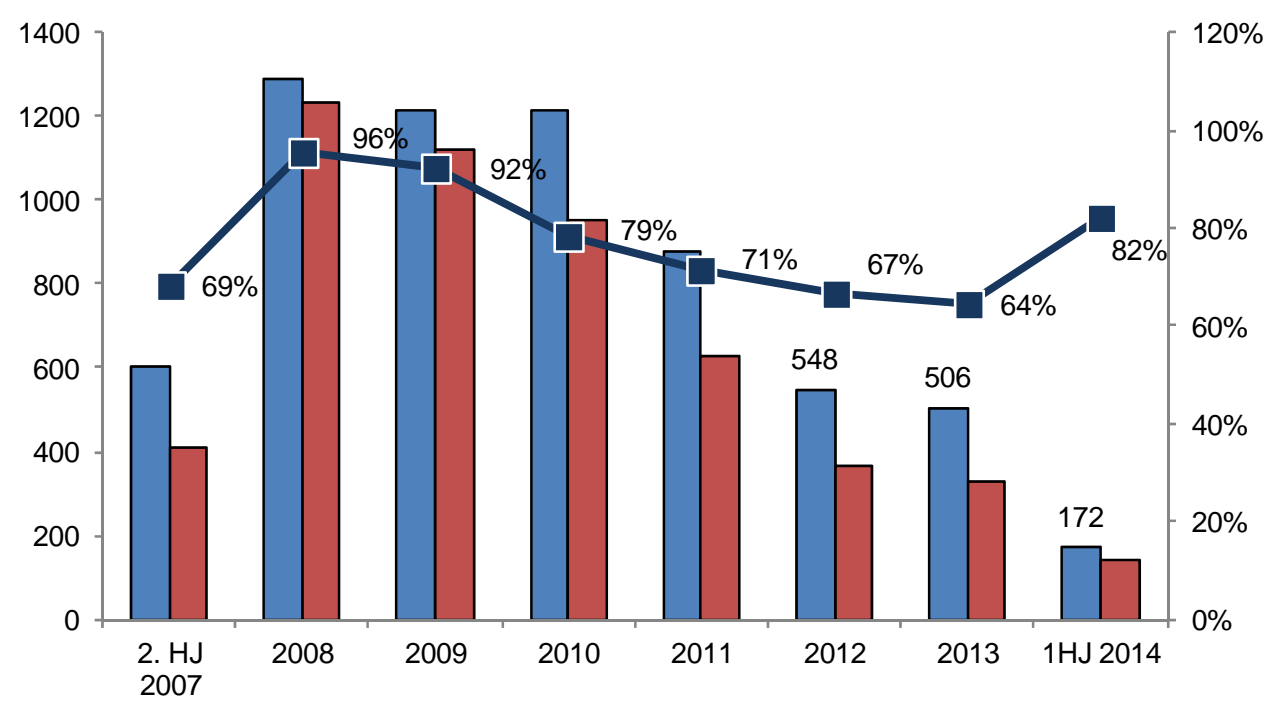

$\square$ Zahl der Förderanträge (Einreichungen)

Genehmigungsquote

Quelle: FFG Monitoring des Bereichs Basisprogramme 
Wenig überraschend stammt der Großteil der partizipierenden Unternehmen aus Wien (26\%), der Steiermark (24\%) und Oberösterreich (19\%). Gegenüber der Zwischenevaluierung des kleinen Innovationsschecks fällt auf, dass die niederösterreichischen Unternehmen seltener einen ISplus in Anspruch nehmen als den IS. Der Anteil an durch den ISplus erstgeförderten Unternehmen ist in der Steiermark unterdurchschnittlich, insbesondere in Ober- und Niederösterreich hingegen überdurchschnittlich (siehe Grafik 13 im Anhang). Darüber hinaus ist ein Viertel der geförderten Unternehmen den Branchen der industriellen Produktion zuzurechnen, 15\% stammen aus dem Bereich der IKT und weitere $13 \%$ sind vorranging als Großhändler aktiv. Diese Verteilung ähnelt jener, die bereits für den kleinen Innovationsscheck festgestellt werden konnte.

Gemäß den Förderkriterien des ISplus handelt es sich bei den untersuchten Fördernehmern ausschließlich um kleine und mittlere Unternehmen. Letztere wickelten rd. 15\% der relevanten ISplus Projekte ab; der Anteil an Kleinstunternehmen mit weniger als zehn Mitarbeiterlnnen von rd. 50\% ist erwartungsgemäß hoch (siehe Grafik 14 im Anhang). Angesichts dieser Zahlen scheint der Anteil von 20\% der Unternehmen, die nach 2009 gegründet wurden, beinahe etwas niedrig.

Die 334 Beteiligungen von Forschungseinrichtungen lassen sich in Teilnahmen von Universitäten, außeruniversitäre Forschungseinrichtungen und Fachhochschulen gliedern. Im Vergleich zum IS €5.000,- (UNI: 31\%, AUF: 47\%, FH: 20\%; siehe Evaluierung durch Good und Tiefentaler 2011) können wir feststellen, dass der ISplus von einem höheren Anteil an Universitätsinstituten durchgeführt wird. 


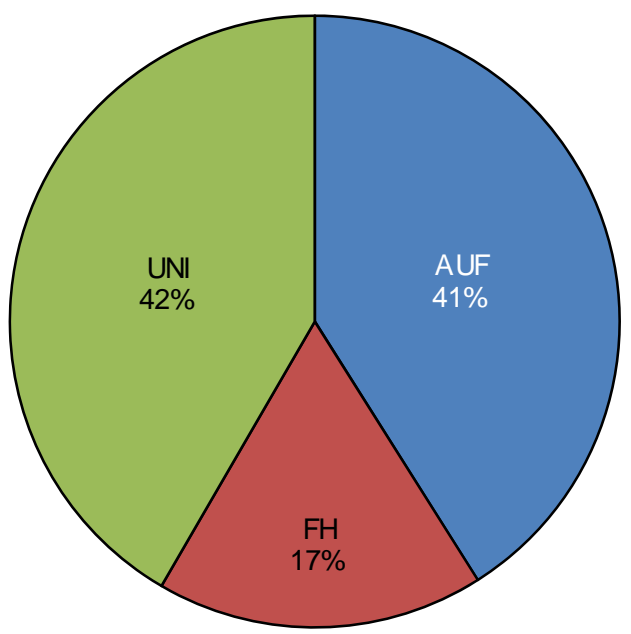

Quelle: Auswertung der FFG Datenbank per 17.10.2014; abgeschlossene Projekte; der Abgrenzungszeitraum der Evaluierung wurde mit 30.6.2014 gewählt, damit drei volle Jahre des Programms ISplus analysiert werden konnten. UNI = Universitätsinstitute; AUF = Außeruniversitäre Forschungsinstitute; FH = Fachhochschule

Um eine erste Annäherung für Folgeaktivitäten von Unternehmen mit ISplus Projekten zu erhalten, wurden die FFG Monitoringdaten nach unterschiedlichen Unternehmenstypen klassifiziert und deren Aktivitäten im FFG Portfolio ausgewertet. Aus folgender Tabelle, sowie den beiden Tabellen 2 und 3 im Anhang, können wir schließen, dass jene ISplus Nutzer, die auch längerfristige FFG Kunden sind sowie jene Unternehmen, die zwar erst nach 2005 in das FFG Portfolio einstiegen, dies jedoch nicht über die Innovationsschecks machten, durchaus die ganze Breite des FFG Portfolios zu nutzen wissen. Zwischendurch wurden auch IS und ISplus Projekte umgesetzt, wobei nach dessen Einführung schwerpunktmäßig nur mehr das Programm ISplus genutzt wurde. Dies hat u.a. damit zu tun, dass die FFG jene Unternehmen auf das Programm ISplus verwies, wenn sich eine Projektidee als nicht passend bzw. zu klein für das Basisprogramm herausstellte. Da es zwischen den ISplus Projekten und den restlichen Projekten des FFG Portfolios zwar vielfältige, aber nicht notwendigerweise zeitlich aufeinander abgestimmte Folgewirkungen der ISplus Projekte gibt, wird hier kein Bezug zum restlichen Portfolio hergestellt. 
Bei der folgenden Kategorie der Erstförderer ist dieser zeitliche Konnex jedoch vorhanden. Durch den ISplus erstmals geförderte Unternehmen haben bereits innerhalb von drei Jahren nach Programmstart zu knapp 9\% Basisprogrammprojekte, zu über 7\% im Strukturprogramm, und immerhin noch zu rd. 5\% in den thematischen Programmen erfolgreiche Projektanträge gestellt: dies summiert sich auf eine ,Folgeaktivitätsrate' von knapp $24 \%{ }^{13}$ Jene Unternehmen, die über die Programmfolge IS und unmittelbar darauffolgend ISplus in das FFG Portfolio einstiegen, zeigen ein ähnliches Muster, mit einem noch stärkeren Fokus auf das Strukturprogramm und liegen insgesamt bei einer noch höheren ,Folgeaktivitätsrate‘ von rd. 27\%. Eine Förderkette innerhalb des Bereichs Basisprogramme ist damit in Ansätzen zu identifizieren, inkludiert jedoch selten die Feasibility Studien, und kann darüber hinaus in das breite Angebot der Strukturund thematischen Programme gehen.

Es ist zu ergänzen, dass bei den Erstgeförderten die häufigsten Folgeprojekte im Bereich Strukturprogramm bei Talente (mit Abstand auch FoKo) festzustellen sind.

${ }^{13}$ In der Zwischenevaluierung des Innovationsschecks (Good/Tiefenthaler 2011) wurde festgestellt, dass innerhalb der ersten drei Jahre nach Programmstart 15\% der Unternehmen mit Innovationsschecks weitere Projekte in der FFG beantragten (davon waren wiederum $40 \%$ für einen weiteren Innovationsscheck). Daraus resultiert schließlich ein Anteil von 9\% an Projektanträgen im restlichen FFG Portfolio. 
Tabelle 1 Erfolgreiche Projektanträge von Unternehmen mit ISplus Projekten im FFG Portfolio

\begin{tabular}{|c|c|c|c|c|}
\hline \multicolumn{5}{|c|}{ Projektanträge von Unternehmen - Jahr der Einreichung } \\
\hline & Bereich & Programmlinie & $\begin{array}{c}\text { Summe 2007- } \\
09 / 2014 \\
\end{array}$ & $\%$ \\
\hline \multirow{9}{*}{$\begin{array}{c}\text { längerfristige } \\
\text { FFG Kunden und } \\
\text { Nutzer des FFG } \\
\text { Portfolios }\end{array}$} & \multirow{5}{*}{ BP } & Bridge & 16 & \\
\hline & & IScheck & 94 & \\
\hline & & ISplus* & 116 & \\
\hline & & Feasibility & 28 & \\
\hline & & Basisprogramm & 240 & \\
\hline & ALR & & 12 & \\
\hline & EIP & & 1 & \\
\hline & SP & & 231 & \\
\hline & TP & & 61 & \\
\hline \multirow{10}{*}{$\begin{array}{c}\text { ISplus als Erst- } \\
\text { förderung \& } 5 \\
\text { Wiedereinsteiger } \\
\text { durch ISplus (seit } \\
\text { 2005) }\end{array}$} & \multirow{5}{*}{ BP } & Bridge & 0 & \\
\hline & & IScheck & 4 & \\
\hline & & ISplus* & 217 & \\
\hline & & Feasibility & 5 & $2,3 \%$ \\
\hline & & Basisprogramm & 19 & $8,8 \%$ \\
\hline & ALR & & 0 & $0,0 \%$ \\
\hline & EIP & & 0 & $0,0 \%$ \\
\hline & SP & & 16 & $7,4 \%$ \\
\hline & TP & & 11 & $5,1 \%$ \\
\hline & \multicolumn{2}{|c|}{ Summe an Folgeaktivitäten } & 51 & $23,5 \%$ \\
\hline \multirow{10}{*}{$\begin{array}{l}\text { Förderkette IS } \\
\text { und ISplus als } \\
\text { Erstförderung }\end{array}$} & \multirow{5}{*}{ BP } & Bridge & 0 & \\
\hline & & IScheck & 91 & \\
\hline & & ISplus* & 66 & \\
\hline & & Feasibility & 1 & $1,5 \%$ \\
\hline & & Basisprogramm & 6 & $9,1 \%$ \\
\hline & ALR & & 0 & $0,0 \%$ \\
\hline & EIP & & 0 & $0,0 \%$ \\
\hline & SP & & 9 & $13,6 \%$ \\
\hline & TP & & 2 & $3,0 \%$ \\
\hline & \multicolumn{2}{|r|}{ Folgeaktivitäten } & 18 & $27,3 \%$ \\
\hline
\end{tabular}

Daten: FFG Monitoring per 17.10.2014, Auswertung: KMU Forschung Austria

Legende: ISplus $^{*}=$ Abgrenzung des ISplus per 31.12.2013, um die zeitliche Versetzung eines ISplus Projekts und Folgeaktivitäten in anderen Programmen zu approximieren. Diese\%-Sätze werden relativ regelmäßig jeweils ein Jahr nach der Durchführung des ISplus erreicht. Rechenbeispiel: $51 / 217 * 100=23,5 \%$

Längerfristige FFG Kunden = Unternehmen mit FFG Projekten vor 2005 und jene die nacher, aber nicht über die IS in das FFG Portfolio einstiegen. 
Für mehr als die Hälfte der Unternehmen (rd. 52\%) stellte die Beteiligung am Programm Innovationsscheck Plus eine Erstförderung durch die FFG dar ${ }^{14}$; weitere 14\% hatten vorher lediglich den kleinen Innovationsscheck in Anspruch genommen und könnten, je nach Definition, ebenfalls zur Gruppe der Erstgeförderten hinzugezählt werden. Rund ein Drittel der in Grafik 7 dargestellten Fördernehmer hatten bereits vorhergehende FFG-Projekterfahrung in unterschiedlichem Ausmaß: knapp 20\% weisen sogar mindestens drei abgeschlossene FFG Projekte auf. Damit ist der Neukundenanteil gegenüber der Unternehmensgruppe des Innovationsschecks geringer (rd. 83\%) bzw. ist ein verstärktes Interesse am ISplus auch bei erfahrenen Fördernehmern feststellbar.

\section{Grafik 7 FFG Vorerfahrung der ISplus Unternehmen}

1 FFG Projekt: $7 \%(24)$

2 FFG Projekte: $8 \%(27)$ $>2$ FFG Projekte: $19 \%$ (62)

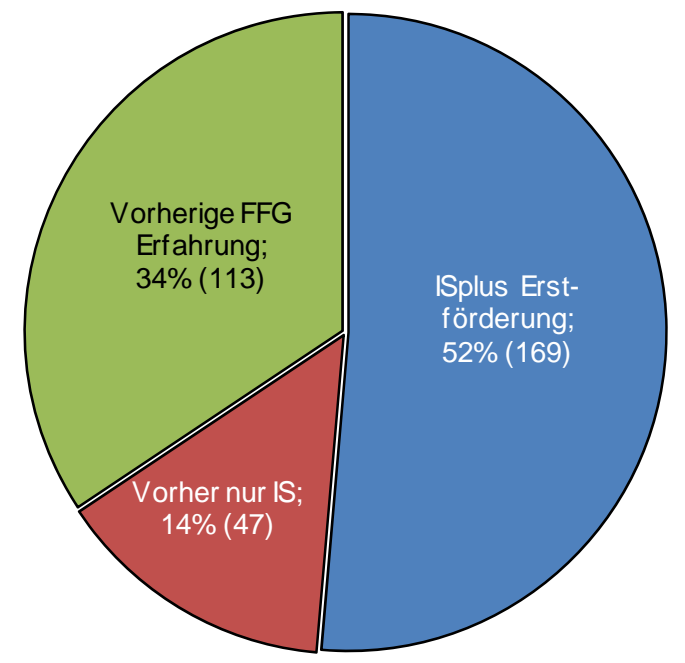

Quelle: FFG Monitoring

Die Zahl der Neueinsteiger hat in der FFG in den Jahren seit Einführung des Innovationsschecks eine deutliche Steigerung erfahren. Lag sie im Jahr 2007 noch bei 289 Unternehmen, so kam es im Folgejahr zu einem Anstieg auf 922 FFG-Neukunden; von diesen nahmen 390 bzw. 42\% einen Innovationsscheck in Anspruch. ${ }^{15}$ Dieser Anteil stieg im Jahr 2009 sogar auf rd. 55\% (550 von

${ }^{14}$ Für fünf Unternehmen bedeutete dies den Wiedereinstieg in das FFG Portfolio, da, gemäß Definition, das letzte FFG Projekt im Zeitraum vor 2005 finalisiert wurde.

${ }^{15}$ Die restlichen Neukunden verteilten sich im Bereich Strukturprogramme schwerpunktmäßig auf COMET, COIN, generation innovation Praktika, später dann auch Talente; im Bereich Thematische Programme verteilt sich dies schwerpunktmäßig sogar auf 7-8 Programme. Im Bereich BP ist die dynamische Entwicklung gänzlich auf die IS zurückzuführen (siehe Grafik 17 im Anhang). 
1.004 Erstfördernehmern). Entsprechend der Nachfrage bzw. dem Verlauf der Bewilligungsquoten des IS reduzierte sich die Zahl der Erstförderungen zwar wieder und betrug im Jahr 2013 schließlich 647 Fördernehmer, dennoch kam es gegenüber dem Ausgangsjahr $2007 \mathrm{zu}$ mehr als einer Verdoppelung der Erstförderungen. Wie bereits erwähnt ist dabei zu berücksichtigen, dass der ISplus die Nachfrage der KMU nach dem IS teilweise substituierte.

\section{Grafik 8 Entwicklung der FFG Erstfördernehmer im Zeitverlauf}

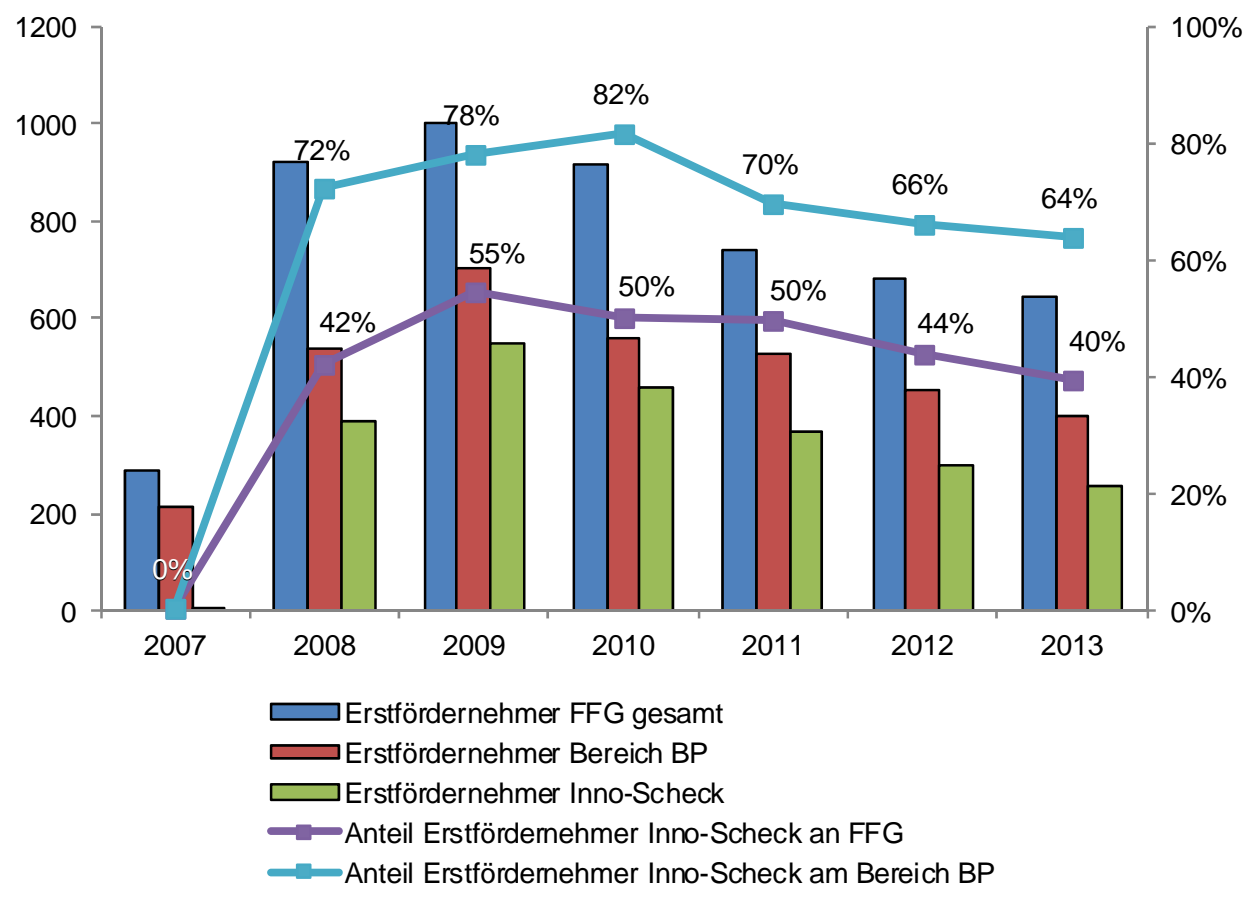

Quelle: FFG Datenbank. Kalkulation: KMFA 


\section{Fazit}

Dies wird wiederum anhand der Beantwortung der jeweils passenden Evaluierungsfragen aufbereitet.

- Werden durch den Innovationsscheck Plus Zielgruppen mobilisiert, die bisher nicht erreicht wurden?

Dies ist der Fall. Über der Hälfte der geförderten Unternehmen wurde mit dem ISplus zum ersten Mal von der FFG unterstützt. Zählt man jene Unternehmen hinzu, die über die Kette kleiner IS und ISplus den Zugang zum Förderportfolio der FFG gefunden haben, so ergeben sich zwei Drittel an Neukunden. Den Programmzielen entsprechend werden damit Eintrittsbarrieren überwunden. Dennoch sind rd. ein Drittel der Fördernehmer Unternehmen, die bereits im Vorfeld FFG-Förderungen erhalten haben (aber nicht notwendigerweise mit einer FE zusammengearbeitet haben), rd. 20\% sogar mindestens drei FFGProjekte, und stellen damit nicht die primäre Zielgruppe des ISplus dar. Diesbezüglich ist auch zu erwähnen, dass Neukunden der FFG in Anschluss an die Projektdurchführung häufiger eine weitere Zusammenarbeit mit der FE anstreben (bereits realisiert oder geplant), als dies für erfahrene FFG Fördernehmer feststellbar ist. Der Wille eine längerfristige Zusammenarbeit mit der betreffenden FE zu etablieren ist daher stärker bei den FFG Neukunden ausgeprägt und unterstreicht eine höhere Kooperationsbereitschaft aufgrund der gemachten positiven Erfahrungen. Ein Teil der Neukunden ist zwar an einer weiteren Kooperation interessiert, aber sie wissen derzeit noch nicht, in welchem Rahmen sie diese weiterführen können.

- Gibt es systematische Übergänge zwischen den beiden Formaten Innovationsscheck und Innovationsscheck Plus?

Die Kette IS zu ISplus haben innerhalb von drei Jahren 47 Unternehmen durchlaufen, das sind $14 \%$ der im ISplus aktiv gewordenen Unternehmen. Allerdings ist dabei zu berücksichtigen, dass es sich nicht ausschließlich um unmittelbare Projektketten handelt, sondern laut Befragten ein beträchtlicher Teil der IS und ISplus Projekte nicht im thematischen Zusammenhang standen. Diese Aussage wird durch den hohen Anteil von Projekten gestützt, deren Grundidee nicht bereits in anderen Projektanträgen verwendet wurde (rd. 83\%). D.h. ein systematischer Übergang vom IS zum ISplus ist nicht unbedingt im Sinne einer linearen Nutzung der Innovationsschecks für eine Projektidee erkennbar. Dabei ist die Rolle der Forschungseinheiten hervorzuheben, die meist einen guten Überblick über die österreichische Förderlandschaft besitzen und die Unternehmen beratend unterstützen. Hier wird oft gleich für den ISplus entschieden, da man mit den daraus finanzierbaren zwei Personenmonaten bereits einen bedeutenden 
Schritt hinsichtlich der Ideenprüfung machen kann, und der Umweg über den kleinen Innovationscheck aufgrund der Förderbedingungen (ein Scheck pro Jahr) oft eine künstliche Pause bedeuten würde.

- Gibt es andere Programme als Alternativen?

Aus den FE und Unternehmensinterviews sowie der Fokusgruppe ist hervorgegangen, dass es nach dem Empfinden der Gesprächspartner keine vergleichbaren, alternativen Programme zu den Innovationsschecks gibt. Im Speziellen erscheint manchen Erstfördernehmern aus innovationsarmen Branchen und KMU ohne ausgewiesene FuE-Mitarbeiter das restliche FFG Portfolio aufgrund der Risiko/Aufwand-Relation als zu weit entfernt von deren betrieblichen Realität. Besonders Unternehmen mit vorheriger Erfahrung mit dem kleinen IS verweisen auf die Komplementarität des ISplus, im Rahmen dessen aufgrund des höheren Budgets ein deutlich höheres Potenzial zugeschrieben wird. Darüber hinaus sind Feasibility Studien bzw. das Basisprogramm mit deutlich höheren Kosten- und Zeitaufwand sowie FuE-Ansprüchen verbunden, die den Fragestellungen des ISplus nicht (unmittelbar) gerecht werden. Die Inanspruchnahme eines alternativen Förderangebots im Falle keiner Förderung durch die FFG wurde dementsprechend auch nur von einem einzigen Interviewpartner als mögliche Vorgehensweise angegeben. Die Feasibility Studies als nächstgelegenes Programm im KMU Paket spielt kaum eine Rolle, und konnte damit seine Funktion als "benachbartes" Folge-Programm, wie im ISpus Programmdokument angedacht und dem KMU Paket visualisiert, nicht wirklich erfüllen.

- Welche Muster an Folgeprojekten der Unternehmen lassen sich innerhalb des FFG-Portfolios bereits abbilden?

Betrachtet man die Unternehmensgruppe der Erstfördernehmer, welche primär mit dem ISplus adressiert werden, so zeigt sich das Basisprogramm als Hauptanlaufstelle für weitere Aktivitäten in der FFG (knapp 9\% der Projekte). Die Unternehmen wurden aber bereits zu über 7\% im Strukturprogramm, und immerhin noch zu rd. 5\% in den thematischen Programmen aktiv: dies summiert sich auf eine ,Folgeaktivitätsrate' von knapp 24\%. Dies ist im Vergleich zum IS $€ 5.000$,-- als sehr positiv zu werten (insgesamt 9\% innerhalb eines vergleichbaren Zeitraums nach Programmstart). Jene Unternehmen, die über die Programmfolge IS und unmittelbar darauffolgend ISplus in das FFG Portfolio einstiegen, zeigen ein ähnliches Muster, mit einem noch stärkeren Fokus auf das Strukturprogramm und liegen insgesamt bei einer noch höheren ,Folgeaktivitätsrate' von rd. 27\%. Eine Förderkette innerhalb des Bereichs Basisprogramme ist damit in Ansätzen zu identifizieren, inkludiert jedoch selten die Feasibility 
Studien, und kann darüber hinaus in das breite Angebot der Struktur- und thematischen Programme gehen.

- Wie hat sich der Anteil der Neueinsteiger in der FFG in den letzten Jahren entwickelt und welche davon sind auf die Kette Innovationsscheck und Innovationsscheck Plus zurückzuführen?

Die beiden Varianten des Innovationsschecks bilden im Rahmen des gesamten Förderportfolios der FFG einen wesentlichen Teil der FFG-Neukunden. Im Jahr 2013 sind z.B. 40\% aller Erstförderungen auf die beiden Innovationsschecks zurückzuführen, und sie stellen rd. zwei Drittel der erstmalig geförderten Unternehmen im Bereich Basisprogramme dar. Insbesondere der Innovationsscheck Plus erweist sich als Zubringer von Erstkunden für das Basisprogramm, und konnte dort einen etwaigen Rückgang von Neukunden auffangen. Darüber hinaus gehen ISplus Neukunden aber auch in das restliche FFG Portfolio - insbesondere in die Strukturprogramme (Talente, etc.), aber auch die Thematischen Programme.

- Wie wird die Abwicklung in Bezug auf Geschwindigkeit, Transparenz des Bewertungsverfahrens, Verständlichkeit der Ausschreibungsunterlagen eingeschätzt?

Die Abwicklung des ISplus wird überwiegend als unbürokratisch bzw. der damit verknüpfte Aufwand als adäquat eingeschätzt. Für Neukunden hat sich der Ablauf teilweise als etwas zeitintensiver bzw. komplizierter dargestellt; es wurde jedoch hervorgehoben, dass die FE im Falle auftretender Probleme unterstützend zur Seite stehen. Bezüglich der Geschwindigkeit der Auszahlung kommen manchmal Anregungen, die insbesondere auf die regelmäßigen im Herbst auftretenden Engpässe in der FFG zurückzuführen sind. Insgesamt entspricht der ISplus aber den Ansprüchen eines niedrigschwelligen Förderinstruments, und wird von der FFG effizient umgesetzt. 


\section{Sicht der Unternehmen}

Dieses Kapitel basiert auf einer repräsentativen telefonischen Befragung von 42 Unternehmen; das sind rd. 13\% der Unternehmen, die in diesem Zeitraum ein ISplus Projekt abschließen konnten. An dieser Stelle werden nur die wichtigsten Grafiken dargestellt; weitere Visualisierungen zu den Ergebnissen der Befragung sind dem Anhang zu entnehmen.

Der Innovationsscheck Plus ist in wesentlichem Maße (zu 83\%) in originäre Projektideen eingeflossen, die bis dato in keinem anderen Projektantrag verwendet wurden. Zu je 31\% wurde der ISplus zum Abtesten einer Produktinnovation eingesetzt, die entweder auf einen geringeren oder hohen Innovationgrad abgezielt hat. Weitere 22\% integrierten das ISplus Projekt in ein bereits laufendes FEI Vorhaben, indem einer spezifischen Frage als Teilaspekt eines größeren internen Projekts nachgegangen wurde.

\section{Grafik 9 Anwendungsbereiche des ISplus}

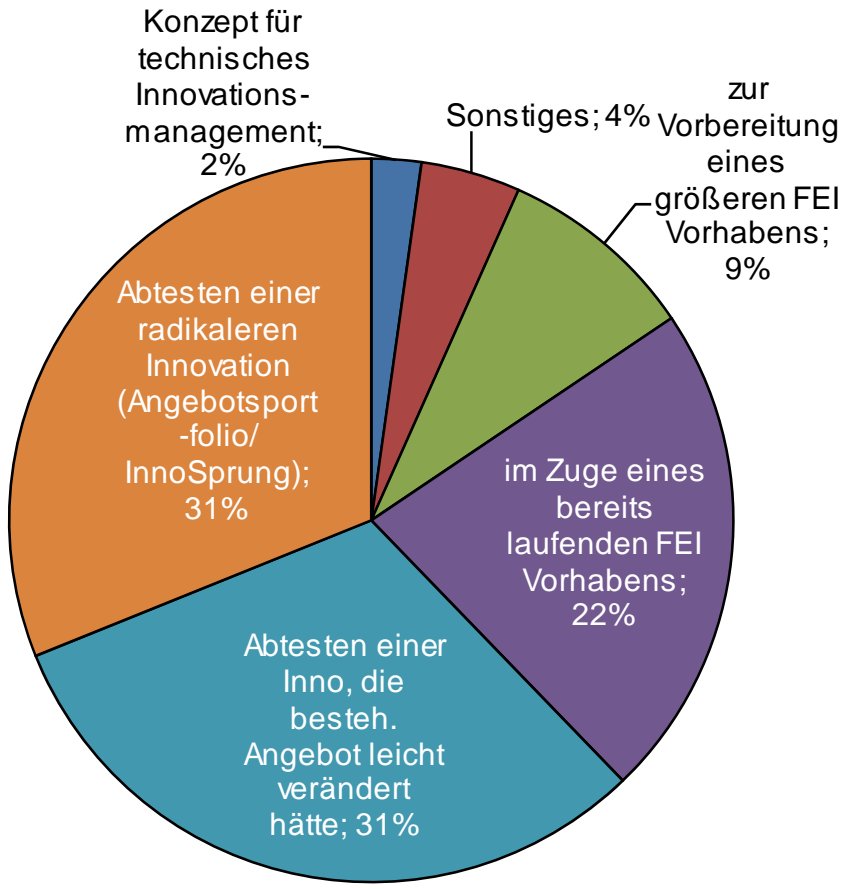

Quelle: Unternehmensbefragung, $n=42$

In Hinblick auf die damit verknüpften Entwicklungsabschnitte von Innovationsvorhaben ergibt sich eine relative Gleichverteilung der geförderten Projekte in der Ideenphase (37\%), der Konzeptphase (34\%), und 29\% der ISplus Projekte wurden bereits in der Prototypenentwicklung eingesetzt. Vier von fünf Unter- 
nehmen ist es durch die Unterstützung der Forschungseinrichtungen gelungen, zumindest eine Phase des Innovationsprozesses zu überspringen bzw. ist es jedem zweiten Unternehmen gelungen seit Projektende mehr als zwei Innovationsphasen hin zur finalen Verwertung der Innovation abzuschließen. Daraus ergibt sich folgendes Bild: 48\% der angestrebten Innovationen können heute bereits wirtschaftlich verwertet werden. Rund ein weiteres Viertel (24\%) der Projektvorhaben befindet sich nun in der Testphase. Keine weiteren Entwicklungstätigkeiten wurden im Zuge von 14\% der geförderten Innovationsvorhaben vorgenommen.

\section{Grafik 10 Heutiger Stand des Projektes und Gesamtprojektkosten}
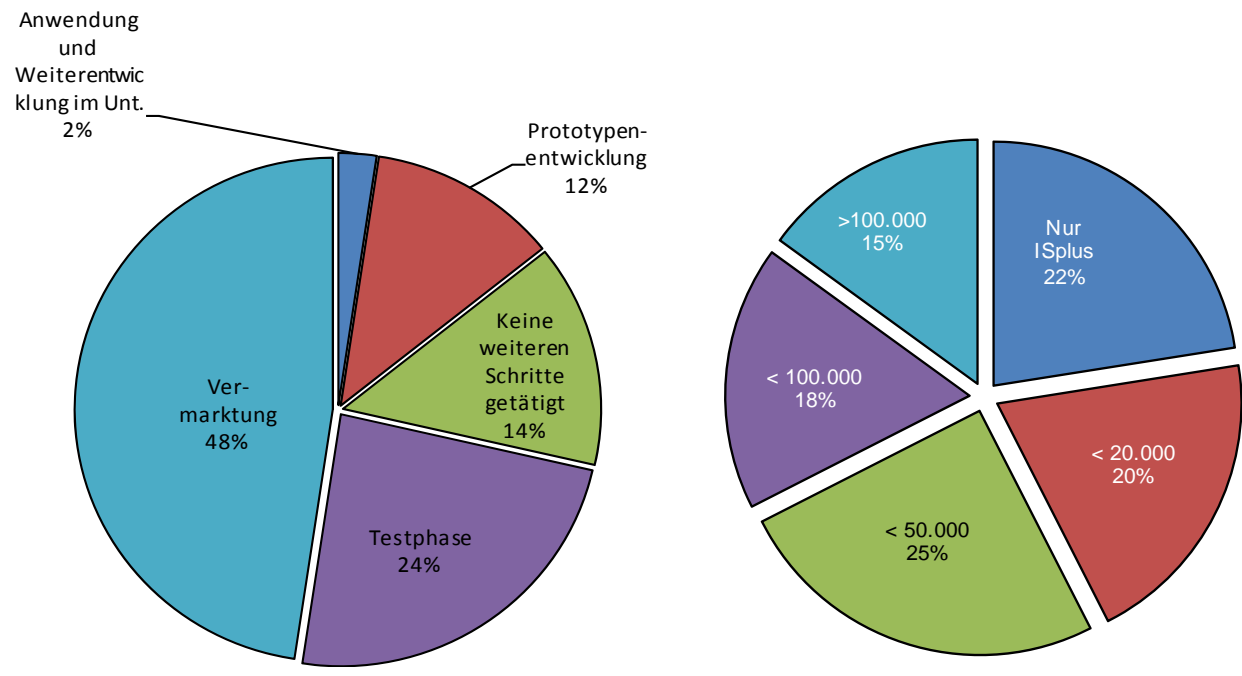

Quelle: Unternehmensbefragung, $n=42$

Bei 22\% der Projekte konnte mit dem Budget des ISplus entweder das Projekt bereits vollständig abgearbeitet werden, oder die ursprüngliche Idee wurde bislang entweder aus technologischen oder wirtschaftlichen Gründen nicht weiterverfolgt. Insbesondere die Projekte im Bereich von $€ 20.000$ bis $€ 50.000$ signalisieren eine hohe Additionalität, da mit dem ISplus Projekt der initiale Schritt zur Projektumsetzung gesetzt wurde. Je größer das Projektvolumen, umso eher sind die ISplus Projekte als ein Teilaspekt von laufenden FEIVorhaben zu interpretieren, und weisen aufgrund dessen geringere Additionalitäten auf; diese Projekte wären auch ohne dem ISplus umgesetzt worden. Aus Grafik 9 lässt sich jedoch auch ableiten, dass immerhin 9\% der Projekte als Vorbereitung von größeren FEI-Vorhaben initiiert wurden.

Wie bereits erwähnt sind rd. die Hälfte der geförderten Unternehmen Erstfördernehmer der FFG, allerdings stellt für lediglich rd. ein Viertel (22\%) der Un- 
ternehmen das Innovationsvorhaben tatsächlich die erste unternehmensinterne Innovationstätigkeit dar. Ausgewiesene FuE-MitarbeiterInnen sind daher in rd. jedem zweiten Unternehmen beschäftigt, wenngleich dies teilweise nur eine Teilzeitperson darstellt; hier kann bereits eine Innovations- und FuE-Affinität unterstellt werden. Des Weiteren haben 62\% der Unternehmen schon vor dem ISplus Projekt mit einer FE zusammengearbeitet, wenngleich dies öfters kleinere Projekte mit dem Charakter „Prüfen und Messen“ waren. Dementsprechend ist die Kooperation zwischen dem Unternehmen und der ISplusForschungseinheit in $24 \%$ der Fälle auf eine vorherige Zusammenarbeit zurückzuführen. Bestand diese nicht, kam es vorwiegend über persönliche Kontakte $(37 \%)$ zu einer Kooperation mit dem Forschungspartner. Hervorzuheben ist darüber hinaus, dass der Anstoß zu der jeweiligen Projektzusammenarbeit beinahe ausschließlich von Seiten der Unternehmen erfolgte. Die konkrete Empfehlung der Inanspruchnahme des ISplus wird aufgrund des Vorschlags der FE nach Abschätzung des Aufwandes gemacht, womit die zentrale Rolle der FE für die Diffusion und Umsetzung der IS unterstrichen wird.

Sowohl der administrative Ablauf als auch die Förderkonditionen des ISplus wurden von den Unternehmen überwiegend positiv bewertet. Hervorgehoben wurde die einfache, schnelle und unbürokratische Abwicklung des Antrages, die das Verhältnis von Aufwand und Leistung des ISplus gut widerspiegelt. Vor allem erfahrene Fördernehmer betonten die Einfachheit des Fördermittels gegenüber umfangreicheren Unterstützungsprogrammen. Die Förderhöhe und der damit verknüpfte Selbstbehalt wurden ebenfalls als angemessen (vor allem für FuE Einsteiger) beurteilt, um die betreffenden Forschungsfragen klären zu können. Etwas kritischer wurde hingegen die 1-Jahresfrist und 5-Jahressperre aufgenommen. Einerseits wurde argumentiert, dass die Weiterführung einer etablierten Forschungskooperation behindert würde (v.a. wenn finanzielle Eigenmittel nur eingeschränkt verfügbar sind), andererseits stellt die Jahresfrist eine künstliche Verzögerung weiterführender FEl-Tätigkeiten dar. Anregungen seitens der Unternehmen bezüglich der künftigen Gestaltung des ISplus waren weiters die Valorisierung der Fördersumme, die Möglichkeit zur Onlineeinsicht des Antragsstatus, die Optimierung des E-calls oder eine verbesserte Darstellung der Kombinierbarkeit von IS und ISplus.

Unabhängig von der Phase des Innovationsprozesses, in dem sich das Innovationsvorhaben heute befindet, konnten 93\% der befragten Unternehmen neues Wissen durch den ISplus erwerben. Darüber hinaus können vier von fünf Unternehmen das neue Wissen auch direkt im Unternehmen einsetzen, wenngleich nur ein Viertel (24\%) der Befragten das erworbene Wissen im Zuge von direkten Folgeprojekten nutzen. Weitere $12 \%$ haben ein weiterführendes Folgeprojekt in Planung. Für einen hohen Anteil von $63 \%$ ist kein unmittelbares Folgeprojekt beabsichtigt; entweder weil die Produktentwicklung bereits abgeschlossen werden konnte $(\sim 50 \%$ der Produktentwicklungen sind bereits im 
Vermarktungsstatus), oder weil sich die Idee als nicht durchführbar herausstellte.

90\% der befragten Unternehmen zeigten sich mit den erbrachten Leistungen der involvierten Forschungseinrichtungen sehr zufrieden bzw. wurden die angestrebten Zielsetzungen vollständig erbracht. Die zufriedenstellenden Innovationsleistungen der FE sind auch ein Ergebnis der intensiven Kooperation zwischen Unternehmen und FE. Sowohl die Zahl der Kontakte zwischen den Beteiligten (wöchentlich bis monatlich) als auch die Qualität (persönliche Treffen, Telefon- und Emailkontakte) empfanden die befragten Unternehmen als durchwegs ausreichend, um den Projektstatus bzw. die weitere Vorgehensweise angemessen zu beurteilen.

Letztlich drückt sich die Zufriedenheit auch in der Fortsetzung gemeinsamer FuE-Tätigkeiten aus. Nahezu zwei Drittel der Kooperationen wurden bereits fortgesetzt bzw. ist diese in Planung (rd. 65\%). Ein leicht abweichendes Verhalten ist hierbei zwischen Neukunden der FFG und erfahrenen Fördernehmern zu erkennen. Neukunden fallen durch eine höhere Bereitschaft auf auch künftig mit der FE zusammenzuarbeiten: nur 28\% der Neukunden haben eine weitere Zusammenarbeit ausgeschlossen (42\% der FFG Erfahrenen).

Die Frage, ob das ISplus Projekt die UnternehmerInnen dazu motiviert hat, in Zukunft generell vermehrt mit Forschungseinrichtungen zusammenzuarbeiten, bejahten zwei Drittel. Allerdings bedeutet das im Umkehrschluss nicht, dass das verbliebene Drittel kein Interesse an einer Zusammenarbeit hat. Vielmehr gaben die betreffenden Unternehmen an, dass auch im Vorfeld des ISplus Projektes bereits mehr oder weniger intensive Kooperationen mit FE bestanden und damit keine höhere Kooperationsneigung aufgrund des Projekts abzuleiten ist. 
Grafik 11 Weitere Zusammenarbeit mit der Forschungseinrichtung

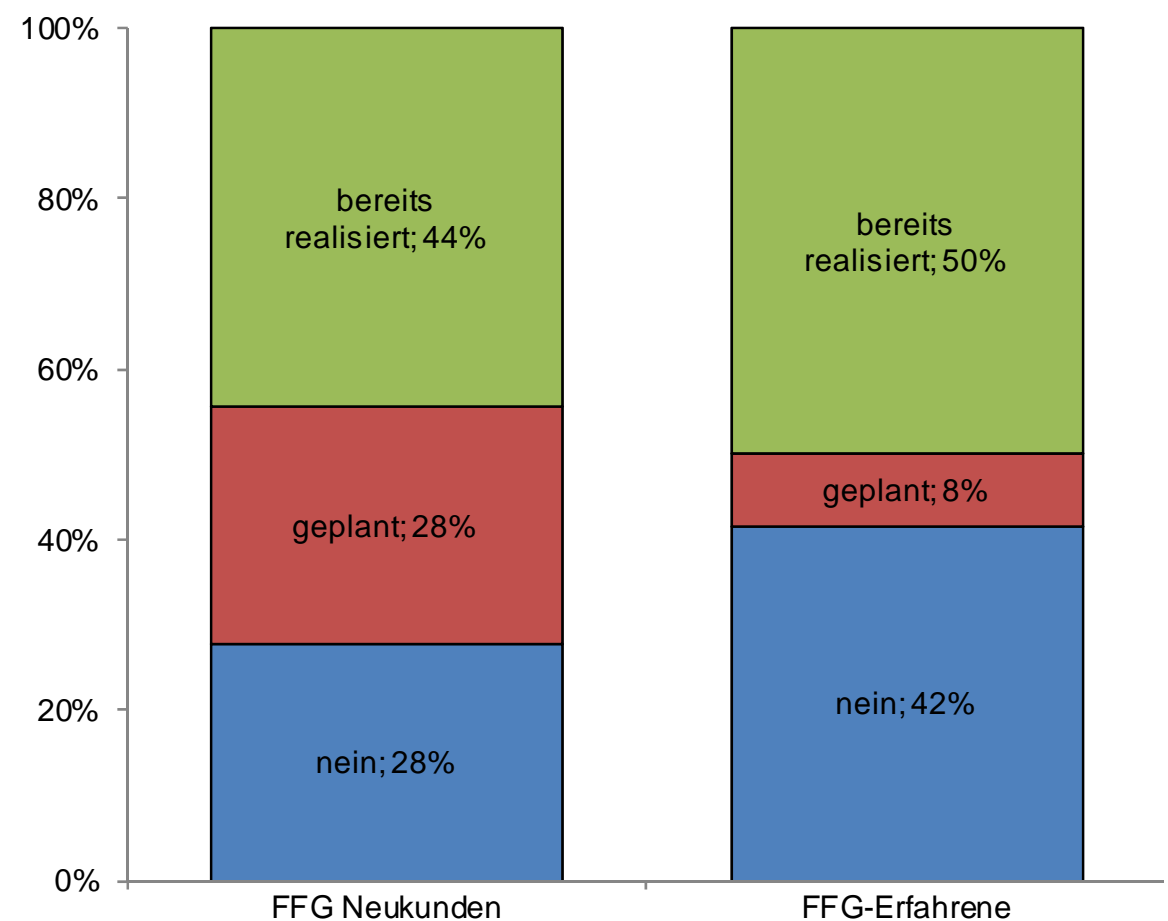

Quelle: Unternehmensbefragung, n = 42

Auf die Frage der Projektadditionalität gaben drei Viertel (rd. 76\%) der befragten Unternehmen an, dass es im Falle keiner Förderung durch die FFG entweder zu einer eingeschränkten (zeitlich verschoben (24\%), geringerer Projektumfang (12\%)) oder gar keiner Durchführung gekommen wäre (40\%). Immerhin $17 \%$ hätten das Innovationsvorhaben auch ohne Förderung selbst finanziert. Die vollständige Finanzierung der Projekte mit eigenen Mitteln wurde vor allem von größeren Unternehmen (MU) und solchen mit umfangreicher FuEErfahrung angegeben.

Aus den Resultaten der Unternehmens- und FE-Befragungen lässt sich eine Typologie der einreichenden ISplus Unternehmen ableiten. Vier primäre Unternehmensgruppen sind dabei erkennbar, die divergierende strukturelle Charakteristika aufweisen und auch unterschiedliche Ergebnisse durch die Förderteilnahme erwarten. Zum einen hat die Befragung gezeigt, dass im ISplus ein nicht unwesentlicher Anteil von Unternehmen aktiv ist, die bereits im Vorfeld durch die FFG gefördert wurde und für die FuE ein Teil der (mehr oder weniger) regelmäßigen Unternehmenstätigkeit darstellt. Es stellt sich die Frage, ob dieser Unternehmenstypus eine Zielgruppe des ISplus bilden soll, bzw. ob diese Unternehmen durch das restliche FFG Portfolio bedient werden sollten. 


\section{Grafik 12 Typologie der erreichten ISplus Unternehmen}

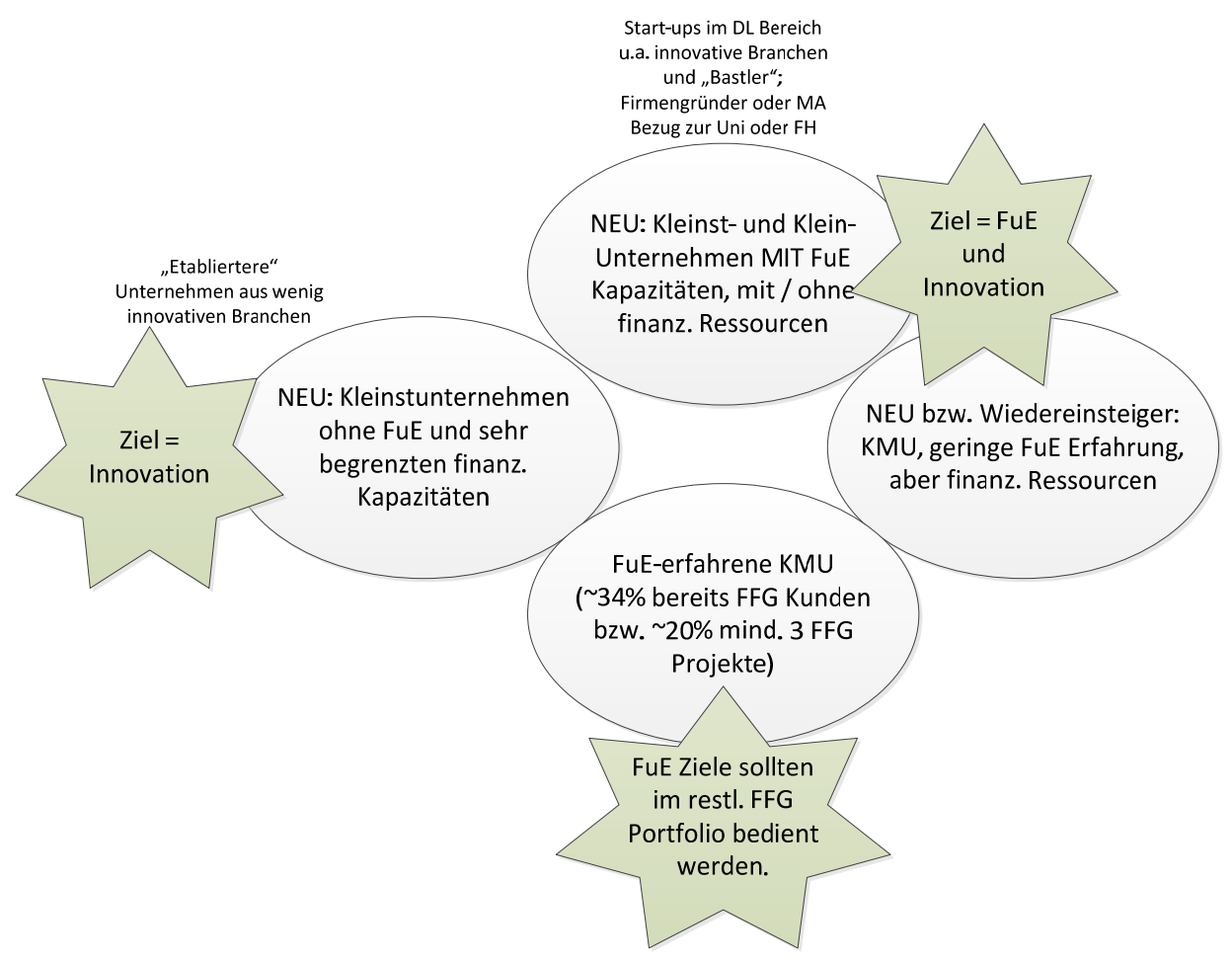

Quelle: KMU Forschung Austria

Die verbleibenden drei Typen von KMU stellen Neukunden bzw. einige wenige FFG Wiedereinsteiger dar. Wesentliche Unterschiede innerhalb dieser Unternehmensgruppen sind einerseits die FuE Affinität und andererseits die finanziellen Ressourcen, die für Innovationstätigkeiten aufgebracht werden können:

- Auf Grund der geringen Innovationsneigung der Unternehmen aus eher traditionellen, wenig innovativen Branchen lassen sich die zugehörigen $\mathrm{KU}$ als „Innovations-Neulinge“ mit wenig FuE Potenzial und geringen finanziellen Ressourcen zusammenfassen. Vorrangiges Ziel der ISplus Projektteilnahme ist die Erreichung einer Innovation bzw. die Lösung einer Problemstellung, für die das interne Wissen fehlt und die ohne größere Entwicklungsschritte im Unternehmen umgesetzt werden können.

- Demgegenüber stehen KU mit ausdrücklichen FuE Kapazitäten, die allerdings in Hinblick auf ihre finanziellen Kapazitäten weiter differenzierbar sind. Der Typus teilt sich daher einerseits in Start-Ups innovativer Branchen und dem Dienstleitungsbereich, die sich aus einer Projektidee entwickelt haben bzw. für diesen Zweck gegründet wurden. Andererseits umfasst dieser Typus Unternehmen, die sich aus dem Forschungsumfeld gebildet haben und aufgrund von bestehenden Bezie- 
hungen ihre MitarbeiterInnen überwiegend aus dem Forschungsbereich rekrutieren. Ein Kennzeichen dieser $\mathrm{KU}$ ist ihre initial hohe FuEOrientierung bzw. bereits umfassende Erfahrungen im FEI-Bereich, die einen wesentlichen Kernbereich der Unternehmenstätigkeiten darstellen. Das angestrebte Ziel der ISplus Beteiligung für diese Unternehmensgruppe ist daher nicht nur die Innovation selbst, sondern auch ihr Aufgreifen in weiteren, internen FuE Vorhaben.

- Letztlich ist eine Gruppe von KMU erkennbar, die geringe Erfahrungen im Bereich der FuE aufweisen, jedoch durchaus Ressourcen für FEI zur Verfügung stellen können. Neben Erstfördernehmern sind hier auch fallweise (= fünf) Wiedereinsteiger in das FFG Portfolio zu finden. Überwiegend sind diese Unternehmen dem sekundären Sektor zuzurechnen. Für diese Gruppe ist das angestrebte Ziel neben den Innovationsergebnissen auch die Verbreiterung der Wissensbasis für eine etwaige stärkere FuE Orientierung.

\section{Fazit}

- Wofür wird der Innovationsscheck Plus vorwiegend eingesetzt? Welche Phasen des Innovationsprozesses im Unternehmen werden mit dem Innovationsscheck Plus besonders adressiert?

Der ISplus wird überwiegend zum Abtesten von Produktinnovationen eingesetzt, und ersetzt teilweise Projekte des kleinen Innovationsschecks, die jedoch tiefergehend analysiert werden. Dementsprechend wird hier nicht nur die Ideenphase, sondern auch die Konzeptphase (34\%) und die Prototypenentwicklung (29\%) unterstützt. Für einen Teil dieser Vorhaben ist eine inhaltliche Ähnlichkeit zu den Feasibility Studien durchaus erkennbar (Machbarkeit), wenngleich mit einer etwas geringeren FuE Intensität und damit einhergehend niedrigeren Eintrittsbarrieren.

- Welche Wirkungen werden erzielt?

In zwei Drittel der untersuchten ISplus Projekte gelang es zumindest zwei Schritte im Innovationsprozess erfolgreich abzuschließen, die überwiegend auch nach Projektende, mit eigenem Geld, weitergeführt wurden (Inputadditionalität). Immerhin rd. 50\% der ISplus Projekte befinden sich inzwischen in der Vermarktungsphase.

Darüber hinaus sind insbesondere bei FFG Neukunden Wirkungen im Bereich der Verhaltensadditionalität wahrzunehmen. Diese liegen bei FEI-Neulingen beim Abbau der Schwellenangst, und steigender Kooperationsbereitschaft und 
Wissenstransfer, bei FEl-kundigen Unternehmen jedoch nur im Bereich der Stimulierung des Wissenstransfers.

Eine Rate von rd. 24\% an FFG Neukunden, die bereits innerhalb der ersten drei Jahre nach Programmstart im restlichen FFG Portfolio aktiv wurden, kann als sehr zufriedenstellend gewertet werden.

- Wie zufrieden waren die Unternehmen mit den Leistungen der Forschungseinheiten?

Die erbrachten Leistungen der FE wurden dabei fast vollständig als sehr zufriedenstellend betrachtet bzw. kam es laut Interviewpartnern in einigen Fällen sogar zu einer Übererfüllung durch den beteiligten Forschungspartner. Nur in wenigen Ausnahmefällen entsprachen die Forschungsleistungen nicht den erhofften Zielsetzungen bzw. kam es zu zeitlichen oder finanziellen Abweichungen von der ursprünglichen Projektplanung.

- Gibt es Indikationen auf einen Einstieg in kontinuierliche FuE?

Über die bereits aus den FFG Monitoringdaten eruierte „Folgeaktivitätsrate“ von rd. 24\% der Neukunden hinaus, kann aus der Unternehmensbefragung folgendes abgeleitet werden: Von den geförderten Unternehmen wurde angegeben, dass der ISplus teilweise als Einstiegsmöglichkeit in die FuE betrachtet wurde bzw. eine Sensibilisierung bezüglich regelmäßigerer Innovationsvorhaben stattgefunden hat. Immerhin 38\% der Befragten gaben weiters an vor dem ISplus noch nicht mit FE zusammengearbeitet zu haben. Allerdings zeigt sich auch ein hoher Anteil an Unternehmen, die bereits im Vorfeld interne FuE-Tätigkeiten durchgeführt haben oder für die FuE Teil des unternehmerischen Tagesgeschäftes ist. Dies drückt sich unter anderem auch dadurch aus, dass ein Drittel der Fördernehmer bereits FFG-Erfahrungen aufweisen konnte und die Durchführung mehr oder weniger kontinuierlicher FuE-Tätigkeiten daher als wahrscheinlich angenommen werden kann. Bei den Neukunden mit FEIVorerfahrung handelt es sich um relativ junge Unternehmen, spezialisierte $\mathrm{Ni}$ schenanbieter mit FuE-Kapazitäten oder akademische Spin-Offs mit daraus resultierender Nähe zu Universität oder Fachhochschulen, die jedoch teilweise sehr geringe Kapitaldeckungen aufweisen. 


\section{Sicht der Forschungseinheiten}

Das Engagement, die Kompetenz und Bereitschaft zum Wissenstransfer durch die Forschungseinheiten (FE) sind ein wesentlicher Bestandteil eines erfolgreichen ISplus Projekts, und der Diffusion des Förderprogramms insgesamt. Die FE ist der zentrale Akteur in der Vermittlung des Wissens über (1) die Durchführbarkeit der Produktidee, und (2) die Information bezüglich eines möglichen Förderprogramms, das für die spezifische Problemlösung geeignet ist.

Insbesondere FuE unerfahrene Unternehmen wenden sich oft an die Forschungseinrichtungen mit der Intention, dass diese die Umsetzung der Idee, von der Konzeptionierung bis hin zur Prototypenentwicklung, vollständig übernehmen. Dementsprechend sind die ersten Schritte der potenziellen Zusammenarbeit von FE und Unternehmen die Spezifizierung des tatsächlichen FEI Bedarfs, die Abschätzung der Umsetzbarkeit durch den ISplus (was ist bereits vorhanden, wo muss selbsttätig innoviert werden) und die Konfrontation mit den Gesamtkosten, die für eine Umsetzung von der Idee bis zur Vermarktung als wahrscheinlich erscheinen. Vom Umfang reicht die Förderhöhe des ISplus in der Regel nicht aus, um eine Idee zu einem Prototyp bzw. einem Endprodukt zu entwickeln, allerdings ist man durchaus in der Lage Resultate zu erzielen "die mehr als nur Papier" darstellen - es wird an einer konkreten technologischen Problemlösung gearbeitet; ein Teil eines Softwarepakets entwickelt, die Produktion eines Werkzeugteils unterstützt oder eine Rezeptur für ein Produkt, etc.

Laut Einschätzung der FE erfordert eine Produktentwicklung meist eindeutig mehr als durch den ISplus abgedeckt wird. Nur in absoluten Ausnahmefällen kann damit eine nahezu komplette Produktentwicklung abgedeckt werden. Kann sich eine Firma den Selbstbehalt für den ISplus nicht leisten, dann ist aus Sicht der FE auch nicht zu erwarten, dass die nachfolgende Produktentwicklung leistbar wäre. Dementsprechend stellt der Eigenanteil der Förderung eine Schwelle dar, die nur jene Unternehmen überschreiten, die ernsthaft hinter einer Projektidee stehen und/oder über den Umfang des ISplus noch weitere Investitionen tätigen können.

Gegenteilig wird der Innovationsscheck € 5.000,-- betrachtet, dessen Umfang bisher nicht valorisiert wurde und aufgrund dessen jährlich immer weniger für die $€ 5.000$ erbracht werden kann. Er wird als das einzige FFG Instrument für sehr kleine Unternehmen (Einzelunternehmen) angesehen, die gar keine Mittel für FuE aufbringen können. Darüber wird der kleine IS insbesondere für Startups als relevant interpretiert, die sich mit spezifischen Fragestellungen an die FE wenden. Eine mögliche Bündelung von kleinen IS von unterschiedlichen Unternehmen würde hier den Spielraum der möglichen Leistungen durch die FE wieder erhöhen. 
Aus Sicht der FE sind ISplus Projekte vor allem dann interessant, wenn ein Know-how Aufbau in beide Richtungen zu erwarten ist. Das bedeutet zum einen, dass das Unternehmen Resultate erhält, die es dem angestrebten Innovationsziel näher bringt, und zum anderen, dass auch die FE einen Know-How Aufbau durch die Projektumsetzung erzielen können. Die Projektinhalte, mit denen sich Unternehmen an die FE wenden, sind jedoch manchmal eher dem Know-How Transfer zuzurechnen. FuE, die in weiterer Folge zu akademischen Publikationen führen könnten, sind weniger gefragt da dieses Wissen für die Firmen nicht direkt verwertbar ist. In diesen Fällen besteht der Know-How Gewinn für die FE eher im Erfassen von branchenspezifischen Technologietrends und praktischen Problemstellungen von Unternehmen (kurzfristige Roadmaps). Von dieser Art der Schließung einer Wissenslücke können Universitätsinstitute und Fachhochschulen prinzipiell verstärkt profitieren als außeruniversitäre Institute, die oft bereits sehr eng mit der Wirtschaft verbunden sind. Da mit dem ISplus im Vergleich zum kleinen IS vermehrt Universitätsinstitute involviert sind, ist dieser Wissensfluss hier intensiviert. Darüber hinaus erfolgt der Wissenstransfer nicht ausschließlich über die durchgeführten Projekte. Die weiterführenden Beziehungen sind mitunter auch unverbindlicher Natur, z.B. werden die ForscherInnen zu Vorträgen, Workshops, oder Trainings eingeladen, oder bestimmte Fragestellungen werden kurz am Telefon erörtert. Dies stellen weitere Hinweise dar, dass die ISplus Ziele des Abbaus der Schwellenangst sowie der gesteigerten Kooperationsbereitschaft unterstützt werden.

Die Rolle des ISplus als Bestandteil des KMU Förderpakets ist nach Ansicht der FE ein eher theoretisches Konstrukt. Die Vertreter der FE sehen den Bezug von ISplus und Feasibility Studien meist wenig komplementär sondern eher substitutiv; sie zielen auf dieselbe Art von Ergebnissen, allerdings mit unterschiedlichen qualitativen Ansprüchen. Als wesentlich wichtiger wird eingeschätzt, welcher konkrete Weg für die Weiterentwicklungen der Produktidee eingeschlagen werden kann. Hier gibt es einen Teil von Unternehmen, für die die Abwägung von Risiko und Eigenbeitrag des Basisprogramms zu herausfordernd ist.

Die Intensität der Zusammenarbeit bzw. des Wissensaustausches ist während der Projektumsetzung teilweise relativ hoch, da aufgrund der konkreten Entwicklungsleistung von rd. zwei Personenmonaten Zwischenergebnisse besprochen werden, und die darauf aufbauenden weiteren Schritte definiert werden müssen.

Da das finanzielle Risiko der Scheckeinlösung bei den Forschungseinheiten liegt, diese jedoch per Definition nicht gewinnorientiert arbeiten, ist eine Nichteinlösung einem KO-Kriterium gleichzusetzen, und dieser Forscher/diese Forschungseinheit wird in Zukunft kaum mehr einen Innovationsscheck Plus durchführen. Die Administration des ISplus wird von den FE im Allgemeinen als adäquat und unbürokratisch eingestuft. Seitens der Förderbedingungen gibt es jedoch Vorschläge, die zu einer flexibleren Beanspruchung der beiden IS führen könnte. Diese fließen bereits in die nun folgenden Schlussfolgerungen ein. 


\section{Schlussfolgerungen und Handlungsemp- fehlungen}

Nach den in der Evaluationsliteratur geläufigen Kriterien für die Beurteilung von Förderprogrammen, können wir die Ergebnisse dieser Evaluierung folgendermaßen zusammenfassen. Darauf aufbauend werden die Handlungsempfehlungen zur weiteren Erhöhung der Effektivität und Effizienz der Programmumsetzung formuliert.

\section{Relevanz und Kohärenz}

Die Relevanz des Programms leitet sich einerseits von der FTI-Strategie des Bundes hinsichtlich der Verbreiterung der Innovations- und FuE Basis in österreichischen Unternehmen, und andererseits aufgrund der tatsächlichen Entwicklung der Innovationstätigkeit (CIS) und der FuE-Quote in den letzten Jahren ab. Da die darin formulierten Ziele noch nicht erreicht sind, sind auch die Mission und die Ziele des Programms sehr aktuell. Vergleichbare Schlussfolgerungen ziehen auch zahlreiche weitere europäische Länder, die in den letzten Jahren Innovationsschecks (in sehr unterschiedlichen Ausführungen) einführten.

Die Kohärenz der Ziele zueinander ist grundsätzlich gegeben; es bedarf jedoch einer Diskussion der Schwerpunktsetzung. Einerseits wird sehr stark betont, dass in KMU eigene FuE auf kontinuierlicher Basis entstehen soll; der alleinigen Innovationsfähigkeit und -praxis ohne FuE, kommt demgegenüber eine untergeordnete Bedeutung zu. Die Empirie zeigt, dass die tatsächlich erreichten Unternehmen über jene mit tatsächlichen FuE Potenzialen auch einen Unternehmenstyp mit Innovationspotenzial, aber kaum FuE Potenzial umfasst. Diese scheinen uns jedoch mittels eines "Innovationsschecks“ ebenso unterstützenswert. Nicht jedes KMU mit Innovationsbedarf muss/soll FuE-Aktivitäten (regelmäßig) setzen, aber jedes Unternehmen sollte anstehende Innovationen umsetzen können, um sich am Markt für die Zukunft positionieren zu können.

Weiters wird im Programmdokument auf Projekte mit, größerem Umfang und höherer Komplexität' [als mit dem kleinen Innovationsscheck] abgezielt. Nicht zuletzt weil der kleine Innovationsscheck seit seiner Einführung nicht valorisiert wurde, kann im Rahmen dessen immer weniger Leistung erbracht werden, und Unternehmen wie auch Forschungseinheiten greifen vermehrt auf den Innovationsscheck Plus zu. In der Empirie weisen die im Innovationsscheck Plus verfolgten Projekte oft den Komplexitätsgrad des kleinen Innovationsschecks auf, die jedoch aufgrund des höheren Volumens weiter vorangetrieben werden und aufgrund dessen eine fundierte Entscheidungsgrundlage für die weitere Vorgehensweise innerhalb der entsprechenden Unternehmen darstellen. Hier bedarf es somit einer klareren Rollenzuweisung zwischen Innovationsscheck und Innovationsscheck Plus, die insbesondere im Rahmen einer gemeinsamen ex- 
post Evaluation geprüft werden sollte (da dies nicht Ziel dieser Zwischenevaluierung war).

Grundsätzlich könnte man sich jedoch zwei unterschiedliche Vorgehensweisen vorstellen. Sollten reine Innovationsprojekte ebenso im Zuge eines Innovationsscheck Plus bedient werden, dann sollten diese in das Zielsystem expliziter aufgenommen werden. Alternativ dazu könnte man auch erwägen, ob die Innovationsscheck Plus Projekte nur für Unternehmen mit FuE Potenzial vorbehalten bleiben sollen, und Innovationsprojekte von Unternehmen mit wenig FuE Potenzial eher mit dem kleinen Innovationsscheck bedient werden. Hier könnte mit der Möglichkeit des Poolens von Innovationsschecks vermehrt an branchenrelevanten bzw. technologiefeld-spezifischen Themen gearbeitet werden. Es stellt sich jedoch die Frage nach einer sinnvollen und einfach administrierbaren Abgrenzung dieser Zielgruppen. Da die Entscheidung über die Beantragung im kleinen oder großen Innovationsscheck entlang der Förderkriterien bereits bislang durch die Forschungseinheiten vorgenommen wurde, könnte eine eindeutigere Formulierung der Förderkriterien eine diesbezügliche Lenkungswirkung erleichtern.

\section{Instrumentenwahl}

Die Wahl des Innovationsscheck Plus, zusätzlich zum kleinen Innovationsscheck, zeigt sich in der Empirie als ein ,Zubringer' von Neueinsteigern für das FFG Portfolio. Der Anteil von FFG Neueinsteigern über den Innovationsscheck Plus, die zu rd. einem Viertel erfolgreiche Projektanträge im restlichen FFG Portfolio stellten, hauptsächlich im Basisprogramm und in den Strukturprogrammen, ist im Vergleich zum kleinen Innovationsscheck sehr positiv zu beurteilen. Für letztere wurde im Zuge der Evaluierung des kleinen Innovationsschecks in einem vergleichbaren Beobachtungszeitraum nach Programmstart ein Anteil von neun Prozent errechnet. Mit dem Innovationsscheck Plus konnte u.a. ein potenzieller Rückgang an Neuantragstellern im Basisprogramm abgefangen werden.

\section{Instrumentendesign}

Mit dem Innovationsscheck Plus werden hauptsächlich Produktinnovationen verfolgt, was durchaus der politischen Intention entspricht. Die Dimensionierung des Innovationsschecks Plus mit $€ 10.000$ plus 20\% Selbstbehalt ist insofern als adäquat einzustufen, als dies rd. zwei Personenmonate an wissenschaftlicher Leistung umfasst, innerhalb dessen ein wichtiger Entwicklungsschritt zur Verfolgung der Produktidee umgesetzt werden kann. Je nach Technologiefeld/Branche lassen sich damit die Phasen des Innovationsprozesses in unterschiedlicher Tiefe abdecken. 
Der Selbstbehalt von 20\% liegt im Ländervergleich im unteren Bereich. Damit zielt man auf Unternehmen mit geringen finanziellen Ressourcen ab, die auch durchaus angesprochen werden können. Es gibt jedoch auch ein Drittel an Unternehmen mit einem unterschiedlich hohen Ausmaß an Vorerfahrung im FFG Portfolio (rd. 20\% sogar mindestens drei FFG Projekte); auf die meisten dieser Unternehmen stellt ein so niedrigschwelliges Instrument mit 80\%-Deckung nicht ab; diese sollten im restlichen FFG Portfolio bedient werden.

Die Fünf-Jahressperre wird seitens der Unternehmen mit wenig FuE-Erfahrung kritisiert, wenn die Zusammenarbeit mit der Forschungseinrichtung gut funktionierte, aber das restliche FFG Portfolio aufgrund des hohen finanziellen Risikos als zu weit entfernt angesehen wird. Andere Länder, wie beispielsweise Nordirland gehen hier weniger restriktiv vor und lassen eine erneute Einreichung mit demselben Forschungspartner zu, wenngleich mit höherem Selbstbehalt für das KMU. Als Alternative könnte man sich vorstellen, für diesen Unternehmenstyp den Zugang zum ,benachbarten` Programm Feasibility Studies zu erleichtern.

Auch die Einschränkung, dass maximal ein Innovationsscheck pro Kalenderjahr beantragt werden kann, wird mitunter bemängelt, da hiermit eine „künstliche“ Pause auferlegt würde. Dies ist auch der Grund, wieso viele Forschungseinheiten und Unternehmen dafür optieren, den Innovationsscheck Plus direkt, ohne den Umweg über den kleinen Innovationsschecks, zu beantragen. Auch hier gibt es Beispiele aus anderen Ländern, die bis zu drei (kleine) Innovationsschecks pro KMU gleichzeitig ermöglichen, sofern diese für unterschiedliche Innovationsvorhaben eingesetzt werden. Eine eingehendere Analyse hierzu konnte im Rahmen dieser Evaluierung nicht durchgeführt werden, und sollte Thema einer gemeinsamen Ex-post Evaluation des kleinen Innovationsschecks und des ISplus sein.

Da das finanzielle Risiko der Scheckeinlösung bei den Forschungseinheiten liegt, diese jedoch per Definition nicht gewinnorientiert arbeiten, ist eine Nichteinlösung einem KO-Kriterium gleichzusetzen, und dieser Forscher/diese Forschungseinheit wird in Zukunft kaum mehr einen Innovationsscheck Plus durchführen. Dies sollte mit besonderer Vorsicht gehandhabt werden, und im Rahmen der Endberichte bei einer nachvollziehbaren Planänderung gegenüber dem im Angebot formulierten Arbeitsplan entsprochen werden.

Die Anforderungen an die Forschungspartner sind zweckmäßig und sollten nicht aufgeweicht werden.

Auch wenn es nicht der Fokus diese Evaluierung war, wurde des Öfteren die Idee eines Poolens von kleinen Innovationsschecks für mehrere Unternehmen andiskutiert, was auch in einigen anderen Ländern erlaubt ist (z.B. in Irland bzw. Deutschland (Bayern)). Aufgrund der sinkenden Nachfrage nach dem kleinen Innovationsscheck könnte dies, abgesehen von einer Valorisierung, 
eine Wiederbelebung ermöglichen. Dies wird den zuständigen Ministerien und der FFG zur Prüfung empfohlen.

Abschließend können wir festhalten, dass die Förderkonditionen den Zielsetzungen des Innovationsschecks Plus grundsätzlich angemessen sind, und mit den oben diskutierten Adaptionen noch effektiver gestaltet werden können.

\section{Vernetzung bzw. Verbundenheit}

Die akademische Literatur wie auch andere Evaluationen weisen darauf hin, dass KMU vor allem in den frühen Innovationsphasen von der Interaktion mit Forschungseinheiten profitieren können, und dass Innovationsschecks am fruchtbringendsten eingesetzt werden, wenn zwischen Wissenschaft und Unternehmen ein (noch) geringer Vernetzungsgrad vorliegt.

Auch die vorliegende Zwischenevaluierung liefert Hinweise darauf, dass die Additionalität bei Unternehmen mit geringen FuE Erfahrungen höher ist, als bei den erfahrenen FFG Kunden. Aufgrund dessen wird eine Schärfung der Zielgruppen vorgeschlagen.

\section{Effektivität und Effizienz}

Der Innovationsscheck Plus ist ein Programm, mit dem der Staat Österreich jährlich mit verhältnismäßig geringem Mitteleinsatz von rd. €2 Mio. bis zu 200 Unternehmen erreicht, und sie bei der Realisierung von überwiegend innovativen Produktideen unterstützt. Im Tandem mit dem kleinen Innovationsscheck (zusätzlich € 2 Mio.) stellt dies einen hoch effektiven Mitteleinsatz dar, solange die intendierten Zielgruppen angesprochen werden können, die ihre Projekte dann auch intern weitertragen.

Der Innovationsscheck Plus hat sich bereits innerhalb der ersten drei Jahre als ein Instrument mit Mehrwert herausgestellt. Er fungiert bereits innerhalb dieser relativ kurzen Frist als „Zubringer“ sowohl für das Basisprogramm, als auch die Struktur- und Thematischen Programme und ermöglicht damit den Einstieg für Unternehmen in das FFG Portfolio. Damit werden Voraussetzungen für eine Verbreiterung der österreichischen Forschungs- und Innovationsbasis geschaffen. Die Beantwortung der Frage, inwiefern damit die Mission des Programms mittelfristig erfüllt werden kann, muss einer zukünftigen ex-post Evaluation überlassen werden.

Insgesamt hat sich die Nachfrage nach den Innovationsschecks seit dem Peak im Jahr 2009 wieder etwas verringert, was mit der höheren Qualitätsorientierung der Administration (und damit höheren Ablehnungsquoten) einherging. Darüber hinaus kann eine Verschiebung vom kleinen hin zum großen Innovationsscheck ab dessen Einführung im Jahr 2011 beobachtet werden, da mit dem 
Innovationsscheck Plus eine tiefergehende Überprüfung der Produktidee erfolgen kann.

Hinsichtlich der angesprochenen Kundengruppen wurde bereits oben auf die FuE-Vorerfahrung der Unternehmen eingegangen, wobei argumentiert wird, dass primär auf Unternehmen mit wenig FuE und wenig FFG Erfahrung abgezielt werden sollte. Branchenmäßig fällt auf, dass bislang ,nur' 25\% der Kunden Industrieunternehmen sind, und damit ein zusätzlicher Kundenkreis für den Bereich Basisprogramme angesprochen wird.

Die administrative Umsetzung des Innovationsschecks Plus seitens der FFG wird als effizient angesehen. Dem entspricht auch die als sehr niedrig einzuschätzende Verfallsquote von $4,8 \%$.

\section{Nachhaltigkeit der Innovations- und FuE-Aktivitäten}

Wir konnten im Rahmen dieser Zwischenevaluierung festmachen, dass deutlich über $20 \%$ an erstgeförderten Unternehmen bereits drei Jahre nach Programmstart im restlichen FFG Portfolio aktiv wurde. Die Frage, ob und inwieweit diejenigen Unternehmen, die sich zum jetzigen Zeitpunkt als Neulinge in der FuEFörderung identifizieren lassen, künftig nachhaltig FuE-Tätigkeiten aufnehmen, kann mit dieser Zwischenevaluierung noch nicht beantwortet werden.

\section{Handlungsempfehlungen}

HE 1: Aufgrund der vorhandenen Datenlage ist das Programm Innovationsscheck Plus positiv zu bewerten. Dementsprechend wird eine Fortführung empfohlen.

HE 2: Eine leichte Adaption der Ziele des Innovationsschecks Plus hinsichtlich der Verankerung des Innovationsbegriffs, wie auch in den anderen Ländern Europas, erscheint erstrebenswert. Dies würde auch eine entsprechende Adaption der im Programmdokument angeführten Indikatoren erfordern, anhand derer die Erreichung der Programmziele überprüft wird (siehe Kapitel 4). Alternativ dazu könnte angedacht werden, den Innovationsscheck Plus für Unternehmen mit FuE-Potenzialen vorzubehalten, und Unternehmen ohne FuE Potenziale mit dem kleinen Innovationsscheck zu bedienen. Hierzu müsste jedoch ein einfaches Beurteilungskriterium gefunden werden, um die administrativen Aufwand gering zu halten. 
HE 3: Die hauptsächlichen Empfehlungen zielen auf die Schärfung des Zielgruppenverständnisses ab:

- Der Innovationsschecks Plus sollte auf Unternehmen mit keiner bzw. wenig FFG Vorerfahrung eingeschränkt werden, da hier die höchste Additionalität zu erwarten ist. FFG-erfahrene Unternehmen sollten mit dem restlichen FFG-Portfolio bedient werden.

- Eine verstärkte Ansprache von Unternehmen des produzierenden Gewerbes / Herstellung von Waren ist überlegenswert, da diese gemäß Community Innovation Survey in den letzten Jahren geringere Innovationstätigkeiten ausweisen. Die Rolle von Intermediären, allen voran den Forschungseinheiten als zentrale Vermittler, aber auch Banken und Steuerberater, Rechtsanwälte, etc. könnten zur Ansprache von potenziellen Industriekunden genutzt werden.

HE 4: Insgesamt scheinen die Förderkonditionen den Zielsetzungen des Innovationsschecks Plus angemessen. Ein Fine Tuning einzelner Förderkonditionen wäre jedoch zu überlegen, wobei dieses allerdings nicht zulasten der Niedrigschwelligkeit des Programms gehen sollte.

- Der Selbstbehalt von 20\% sollte beibehalten werden, wenn die Zielgruppe auf Unternehmen mit wenig FuE-Erfahrung eingeschränkt wird.

- Die Fünf-Jahressperre ist für Unternehmen mit wenig Ressourcen und FuE-Erfahrung ein Hindernis, um eine erfolgreiche Kooperation weiterführen zu können. Dies könnte dahingehend gelöst werden, dass eine erneute Einreichung mit demselben Forschungspartner ermöglich wird, aber mit einem höheren Selbstbehalt für das KMU. Alternativ dazu könnte für diesen Unternehmenstyp der Zugang zum ,benachbarten' Programm Feasibility Studies erleichtert werden. ${ }^{16}$

- Statt der Regel von maximal einem Innovationsscheck pro Kalenderjahr könnte auch eine flexiblere Lösung angedacht werden, wie z.B. zwei Innovationsschecks und ein Innovationsscheck Plus innerhalb von $\mathrm{x}$ Jahren, damit aufgrund der Formalkriterien künstlich verordnete Pausen in den Innovationsaktivitäten der Unternehmen vermieden werden.

HE 5: Es ist anzuraten, in etwa vier Jahren eine Wirkungsevaluierung zur Beantwortung der Frage nach der Aufnahme nachhaltiger FuE bei den heutigen FFG Neulingen aufgrund des Innovationsschecks und des Innovationsschecks Plus durchzuführen. Die beiden Programme sollten zusammen evaluiert werden, um Wechselwirkungen besser erfassen zu können.

\footnotetext{
${ }^{16}$ Während der Durchführung dieser Evaluation fand parallel dazu ein Redesign des Programms Feasibility Studies statt.
} 


\section{Anhang}

\subsection{Weitere Monitoringdaten und Befragungsergebnisse}

Grafik 13 Bundesländerverteilung der Unternehmen und FE

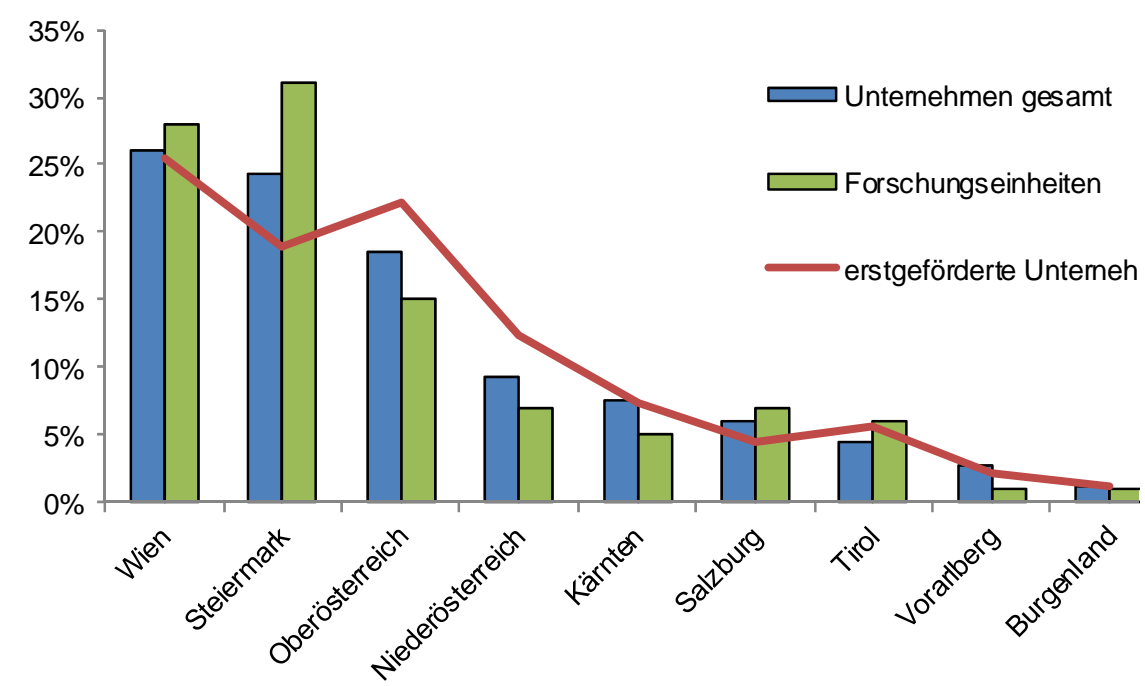

Grafik 14 Größe der ISplus Unternehmen

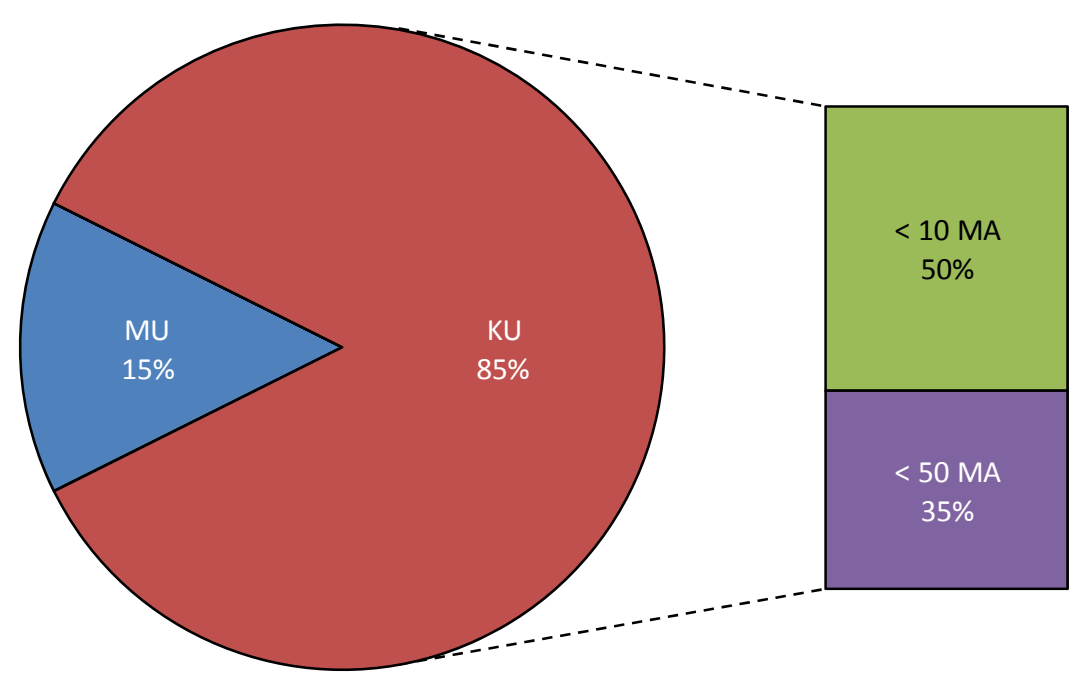


Grafik 15 Programmadministration ISplus

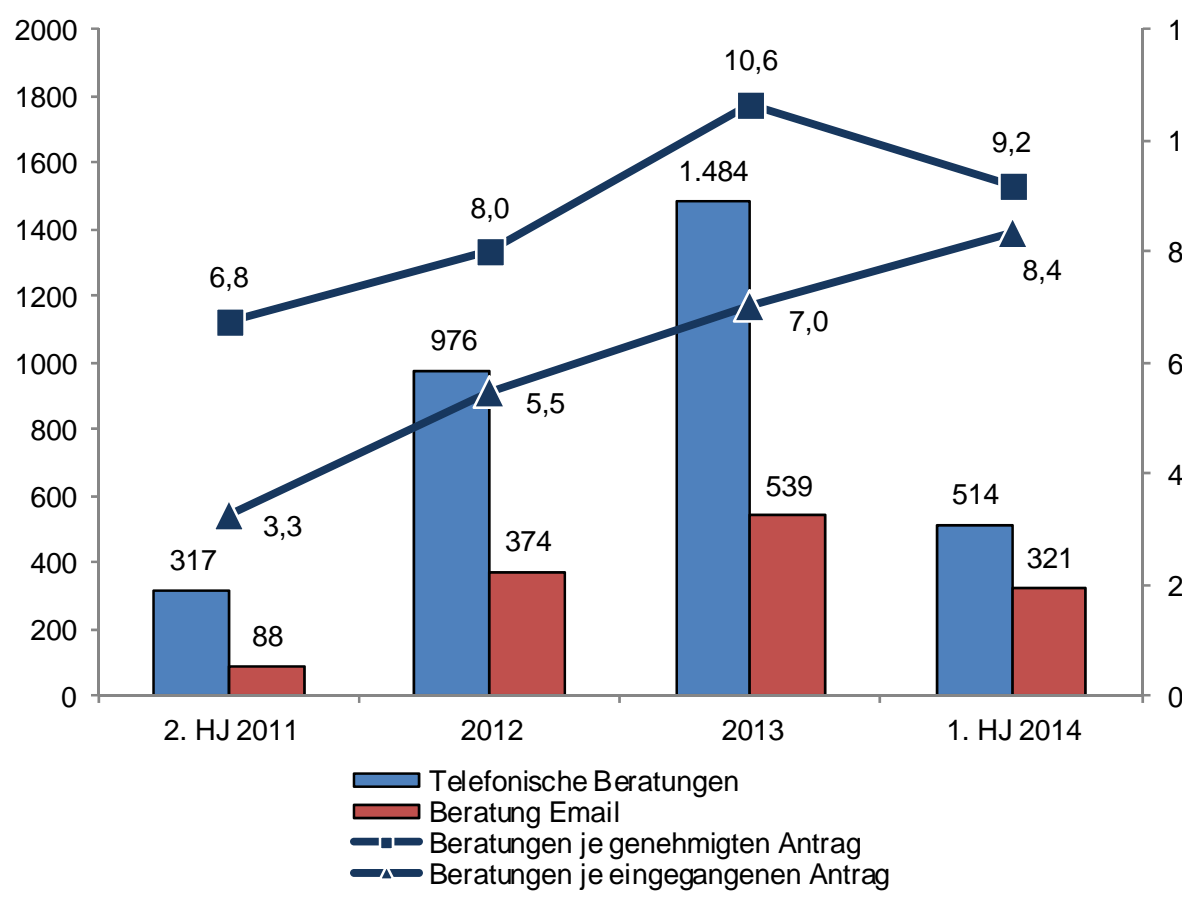

Grafik 16 Erfolgreiche Teilnahme von ISplus Unternehmen in Bereich Basisprogramme, Jahr der Projekteinreichung

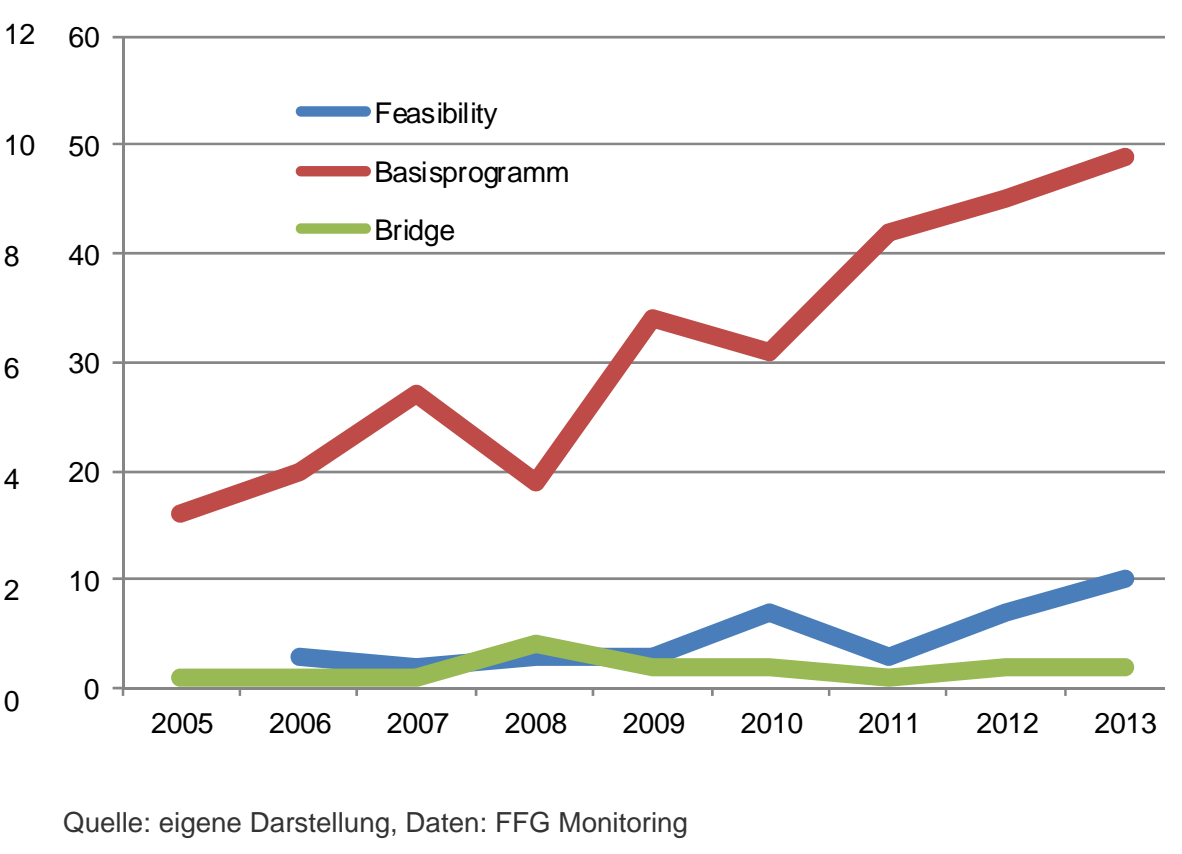

Quelle: eigene Darstellung, Daten: FFG Monitoring 
Tabelle 2 Einfluss des ISplus auf den Bereich BP

\begin{tabular}{|c|c|c|c|c|c|c|c|c|c|c|c|}
\hline & \multicolumn{10}{|c|}{ Unternehmen } \\
\hline & & \multicolumn{10}{|c|}{ Projektanträge - Jahr der Einreichung } \\
\hline & & 2007 & 2008 & 2009 & 2010 & 2011 & 2012 & 2013 & $\begin{array}{c}\text { bis } \\
09 / 2014\end{array}$ & Summe & $\%$ \\
\hline \multirow{5}{*}{$\begin{array}{l}\text { längerfristige } \\
\text { FFG Kunden }\end{array}$} & \multirow{4}{*}{$\begin{array}{l}\text { Bridge } \\
\text { IScheck } \\
\text { ISplus } \\
\text { Basisprogramm }\end{array}$} & 1 & 3 & 1 & 1 & 1 & 1 & & 1 & 9 & $4 \%$ \\
\hline & & 8 & 14 & 4 & 13 & 1 & 3 & 1 & 1 & 45 & $18 \%$ \\
\hline & & \multirow[b]{2}{*}{22} & \multirow[b]{2}{*}{11} & \multirow[b]{2}{*}{14} & \multirow[b]{2}{*}{20} & 8 & 8 & 26 & 7 & 49 & $20 \%$ \\
\hline & & & & & & 17 & 15 & 12 & 12 & 123 & $50 \%$ \\
\hline & Feasibility & 1 & 2 & 1 & 5 & 2 & 4 & 3 & 2 & 20 & $8 \%$ \\
\hline \multirow{5}{*}{ ab 2007 aktiv } & \multirow{4}{*}{$\begin{array}{l}\text { Bridge } \\
\text { IScheck } \\
\text { ISplus } \\
\text { Basisprogramm }\end{array}$} & & 1 & & 1 & \multirow[b]{2}{*}{6} & 1 & 2 & \multirow[t]{2}{*}{2} & 7 & \multirow{3}{*}{$\begin{array}{c}3 \% \\
18 \% \\
33 \%\end{array}$} \\
\hline & & 4 & 2 & 17 & 13 & & 4 & 3 & & 49 & \\
\hline & & \multirow[b]{2}{*}{5} & \multirow[b]{2}{*}{8} & \multirow[b]{2}{*}{17} & \multirow[b]{2}{*}{9} & 17 & 28 & 29 & 17 & 91 & \\
\hline & & & & & & 22 & \multirow[t]{2}{*}{19} & 25 & \multirow[t]{2}{*}{12} & 117 & $43 \%$ \\
\hline & Feasibility & 1 & 1 & 1 & 1 & 1 & & 3 & & 8 & $3 \%$ \\
\hline \multirow{5}{*}{$\begin{array}{l}\text { Erstförderer u. } \\
\text { Wiedereinsteiger } \\
(2005)\end{array}$} & \multirow{4}{*}{$\begin{array}{l}\text { Bridge } \\
\text { IScheck } \\
\text { ISplus } \\
\text { Basisprogramm }\end{array}$} & & & & & \multirow{5}{*}{38} & & & & 0 & $0 \%$ \\
\hline & & & & & & & 1 & 2 & 1 & 4 & $1 \%$ \\
\hline & & & & & & & 84 & 95 & 54 & 271 & $91 \%$ \\
\hline & & & & & & & 3 & 9 & 7 & 19 & $6 \%$ \\
\hline & Feasibility & & & & & & 1 & 3 & 1 & 5 & $2 \%$ \\
\hline \multirow{5}{*}{ nur IS vorher } & Bridge & \multirow{5}{*}{6} & \multirow{5}{*}{13} & \multirow{5}{*}{18} & & & & & & 0 & $0 \%$ \\
\hline & IScheck & & & & 23 & 9 & 14 & 5 & 3 & 91 & $51 \%$ \\
\hline & ISplus & & & & & 12 & 24 & 30 & 12 & 78 & $44 \%$ \\
\hline & Basisprogramm & & & & & & 3 & 1 & 2 & 6 & $4 \%$ \\
\hline & Feasibility & & & & & & & 1 & & 1 & $1 \%$ \\
\hline
\end{tabular}

Quelle: FFG Monitoring; eigene Darstellung; es gibt noch eine Kategorie an Projekten, die zwischen dem IS und dem ISplus andere FFG Förderungen in Anspruch nahmen, die 
nicht dargestellt wird.

Tabelle 3 Einfluss des ISplus auf das restliche FFG Portfolio

\begin{tabular}{|c|c|c|c|c|c|c|c|c|c|c|c|}
\hline & \multicolumn{10}{|c|}{ Unternehmen } \\
\hline & & \multicolumn{10}{|c|}{ Projektanträge - Jahr der Einreichung } \\
\hline & & 2007 & 2008 & 2009 & 2010 & 2011 & 2012 & 2013 & $\begin{array}{c}\text { bis } \\
09 / 2014\end{array}$ & Summe & $\%$ \\
\hline \multirow{4}{*}{$\begin{array}{l}\text { längerfristige } \\
\text { FFG Kunden }\end{array}$} & ALR & 1 & 1 & & & & & 2 & & 4 & $3 \%$ \\
\hline & EIP & & & & & & & & & 0 & $0 \%$ \\
\hline & SP & 7 & 3 & 14 & 6 & 7 & 16 & 17 & 9 & 79 & $55 \%$ \\
\hline & TP & 11 & 9 & 11 & 6 & 4 & 10 & 7 & 3 & 61 & $42 \%$ \\
\hline \multirow{4}{*}{ ab 2007 aktiv } & ALR & 2 & 2 & & & & 1 & 3 & & 8 & $3 \%$ \\
\hline & EIP & & & 1 & & & & & & 1 & $0 \%$ \\
\hline & SP & 4 & 5 & 10 & 15 & 17 & 31 & 39 & 31 & 152 & $64 \%$ \\
\hline & TP & 5 & 11 & 9 & 13 & 9 & 8 & 16 & 6 & 77 & $32 \%$ \\
\hline \multirow{4}{*}{$\begin{array}{l}\text { Erstförderer u. } \\
\text { Wiedereinsteiger } \\
(2005)\end{array}$} & ALR & & & & & & & & & 0 & $0 \%$ \\
\hline & EIP & & & & & & & & & 0 & $0 \%$ \\
\hline & SP & & & & & & 3 & 5 & 8 & 16 & $59 \%$ \\
\hline & TP & & & & & & 1 & 7 & 3 & 11 & $41 \%$ \\
\hline \multirow{4}{*}{ nur IS vorher } & ALR & & & & & & & & & 0 & $0 \%$ \\
\hline & EIP & & & & & & & & & 0 & $0 \%$ \\
\hline & SP & & & & & & 1 & 3 & 5 & 9 & $82 \%$ \\
\hline & TP & & & & & & & 2 & & 2 & $18 \%$ \\
\hline
\end{tabular}

Quelle: FFG Monitoring; eigene Darstellung; es gibt noch eine Kategorie an Projekten, die zwischen dem IS und dem ISplus andere FFG Förderungen in Anspruch nahmen, die nicht dargestellt wird. 
Grafik 17 Anzahl von Erstfördernehmern im Bereich Basisprogramme

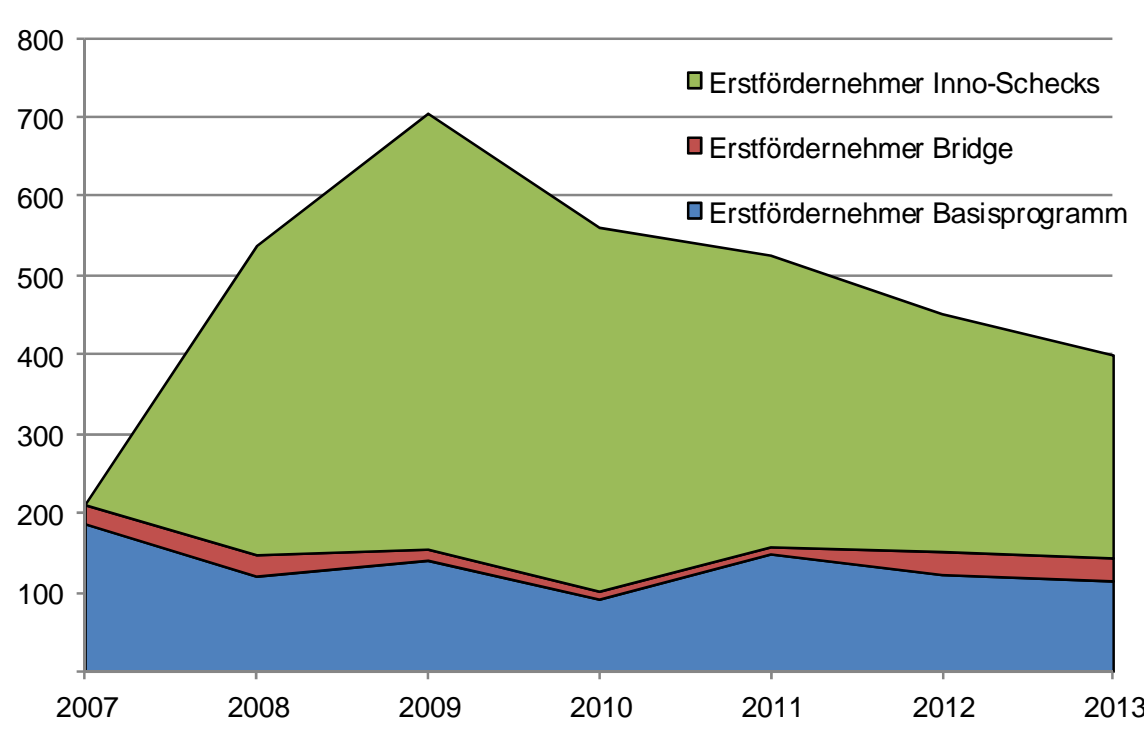

Quelle: FFG Monitoring
Grafik 18 Was hätten Sie getan, wenn Sie keinen ISplus bekommen hätten?

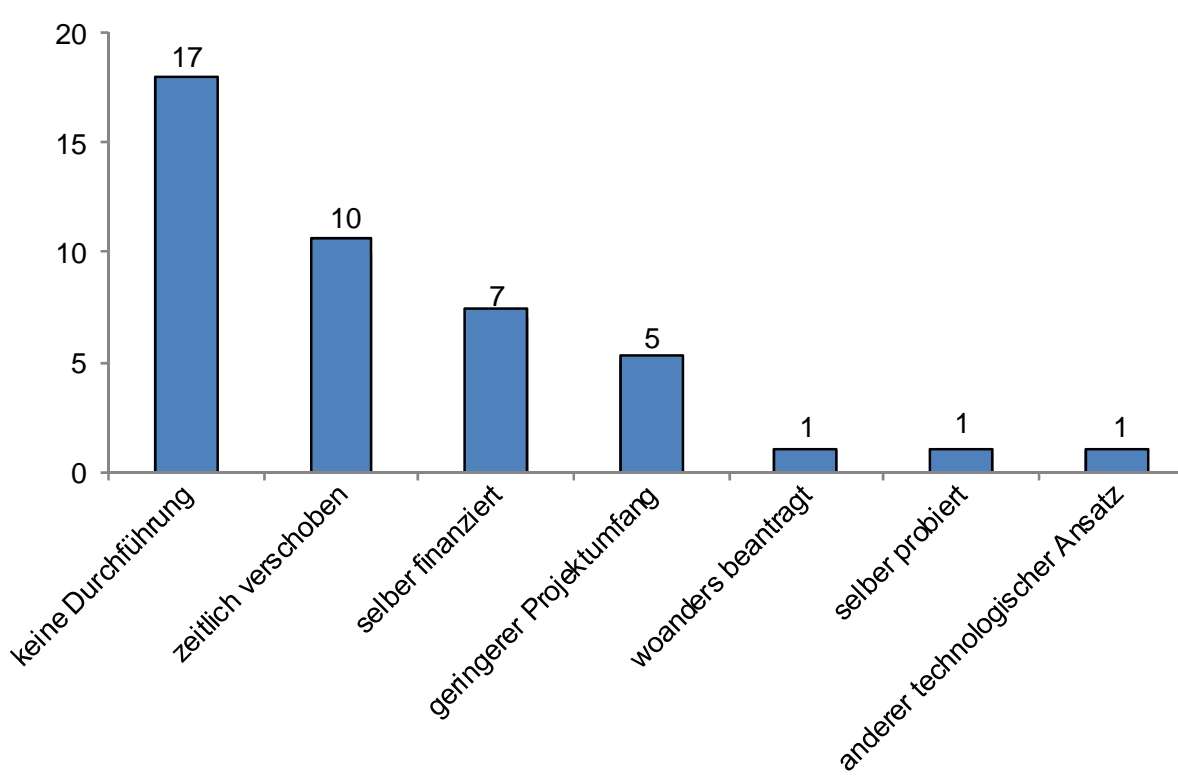

Quelle: Unternehmensbefragung, $\mathrm{n}=42$ 


\section{Grafik 19 Phasen des Innovationsprozesses}

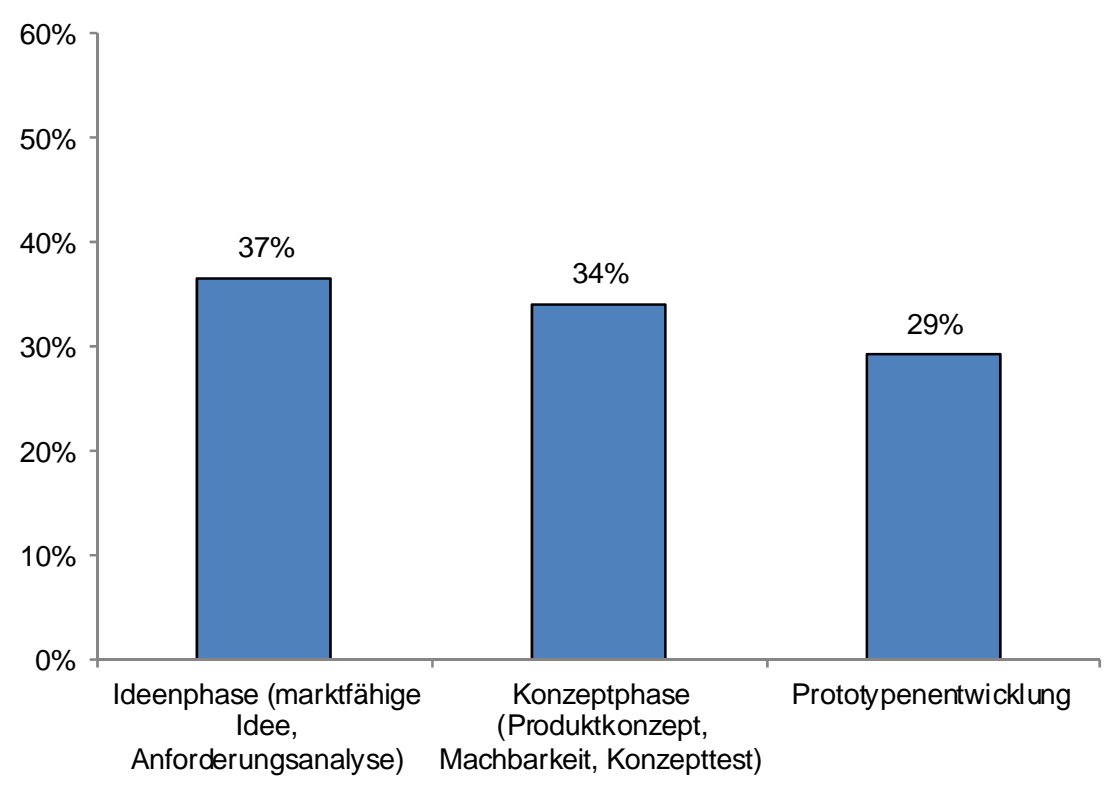

Quelle: Unternehmensbefragung, $n=42$
Grafik 20 Phasen des Innovationsprozesses nach Unternehmensgrößenklassen

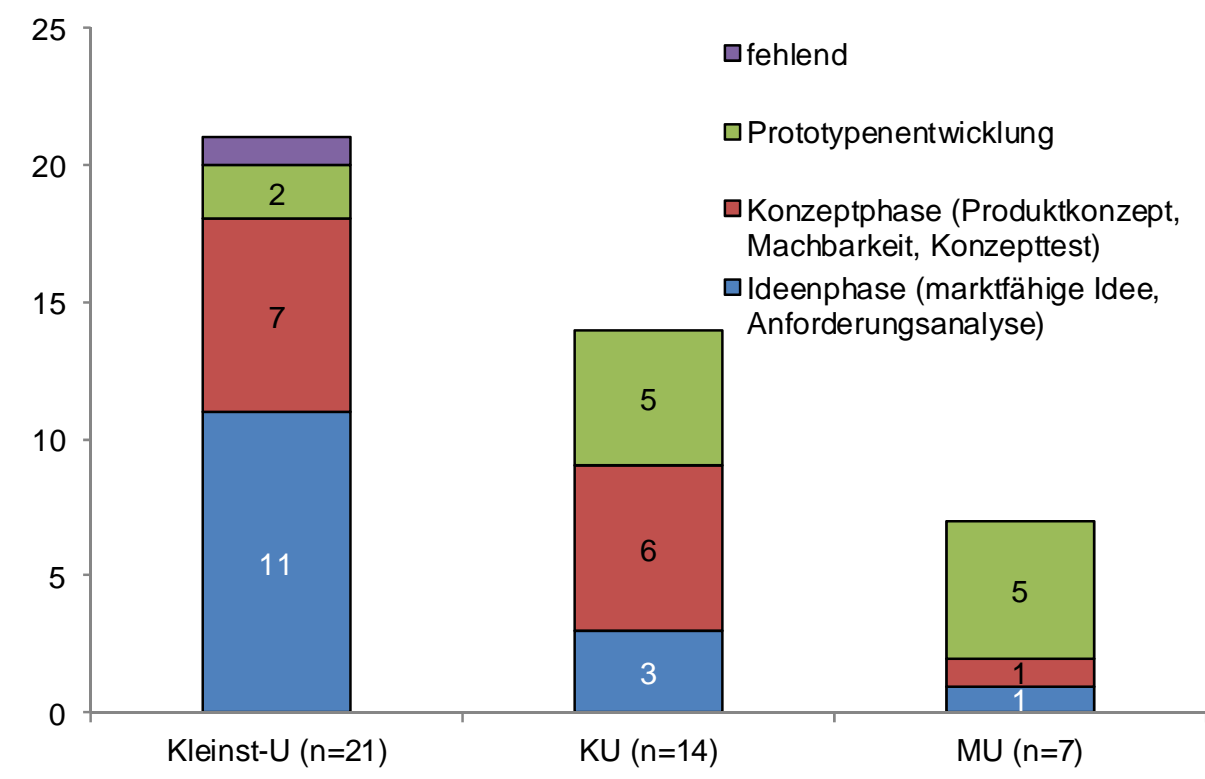

Quelle: Unternehmensbefragung, $\mathrm{n}=42$ 


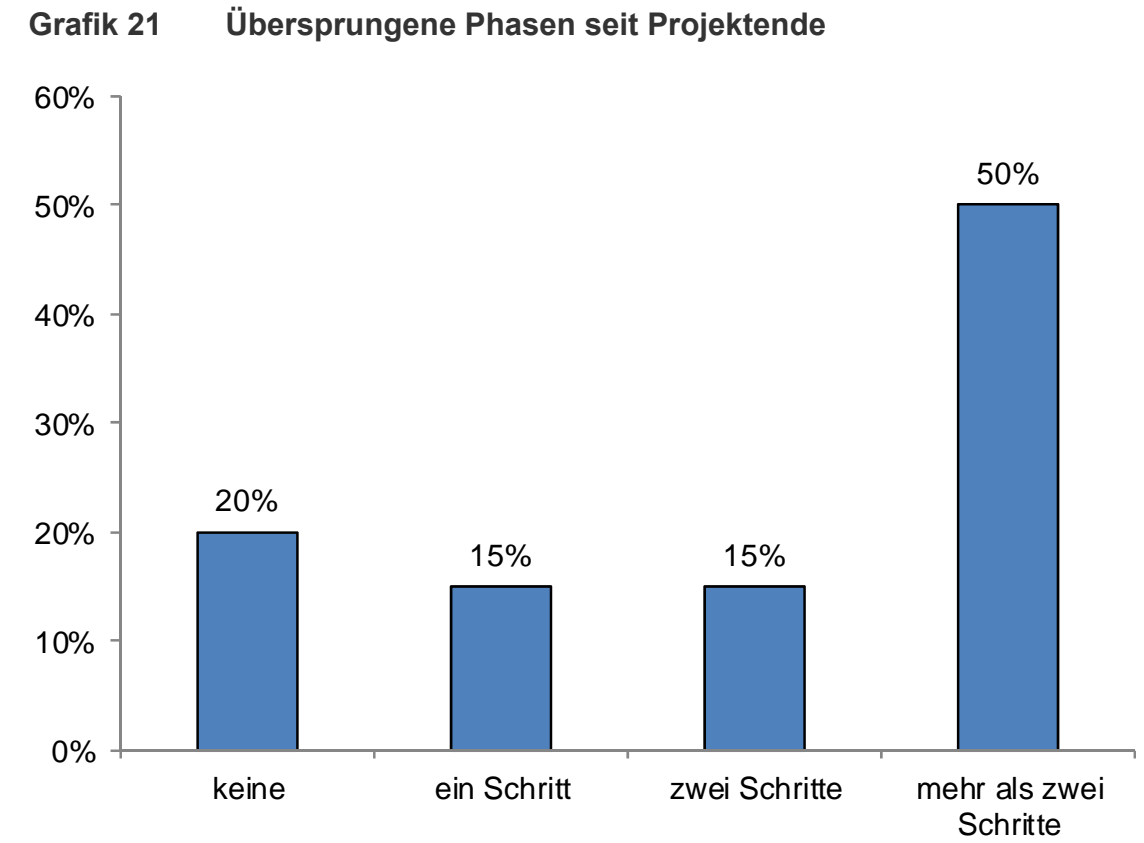

Quelle: Unternehmensbefragung, $n=42$
Grafik 22 Heutiger Stand des Projekts nach Unternehmensgrößenklassen

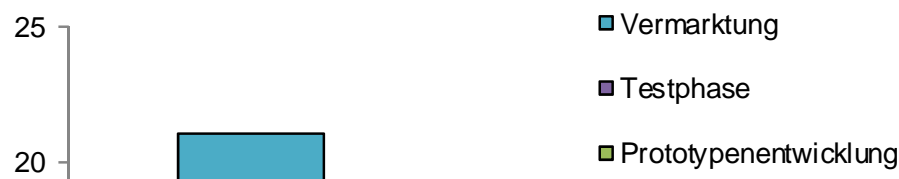

$\square$ Wird im Unternehmen angewendet und weiterentwickelt

口Keine weiteren Schritte getätigt

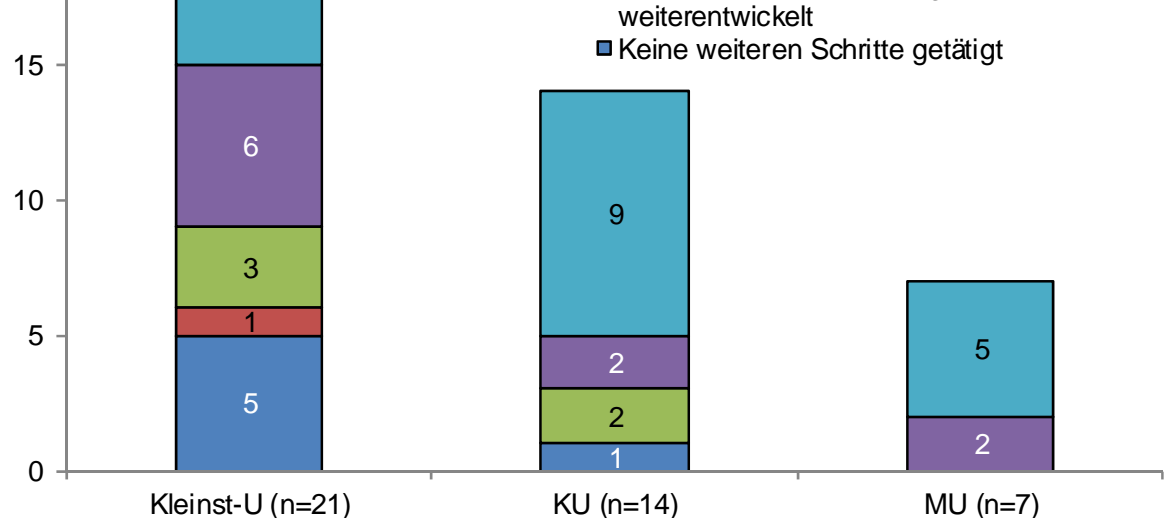

Quelle: Unternehmensbefragung, $n=42$ 


\section{Grafik 23 FuE-Affinität der Unternehmen}

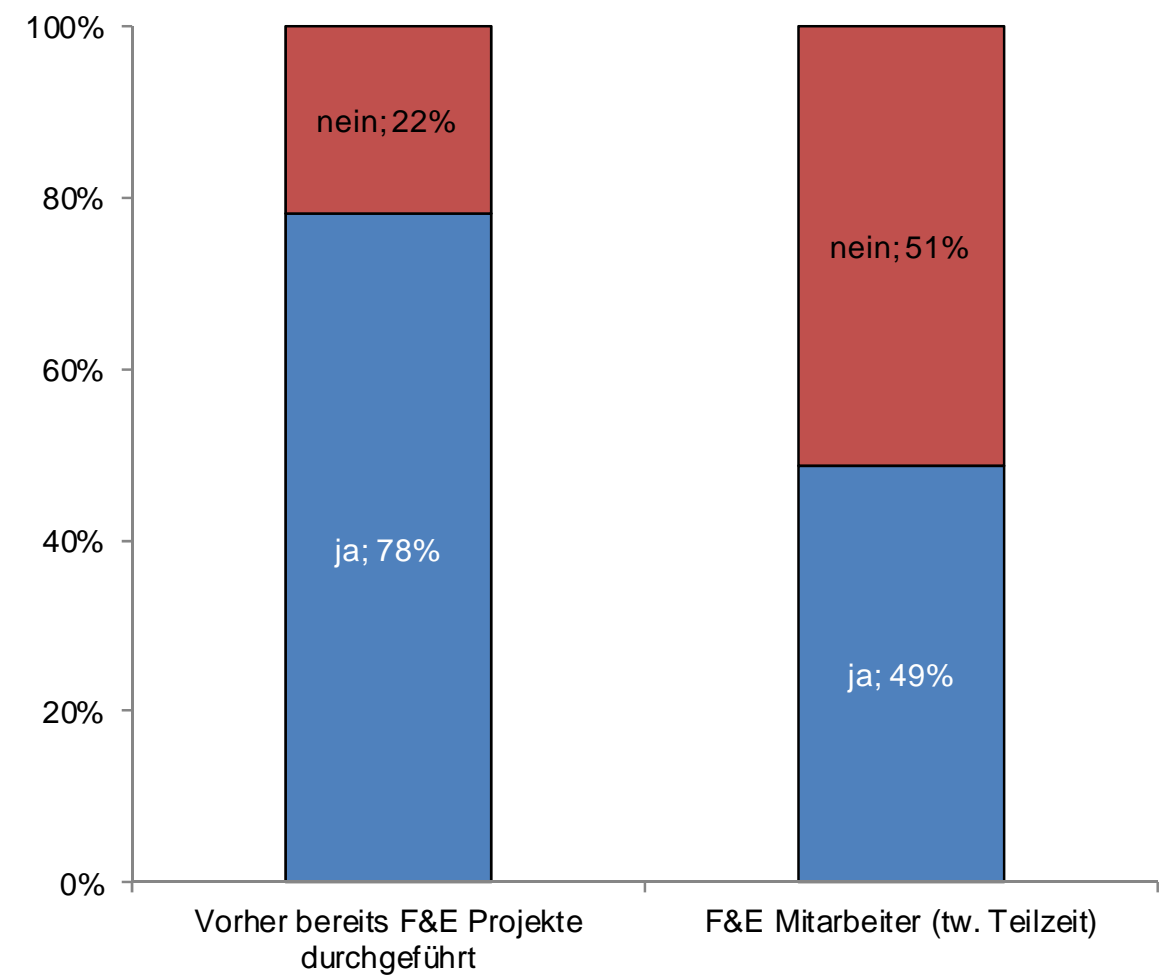

Quelle: Unternehmensb
Grafik 24 Vorherige Zusammenarbeit mit einer FE

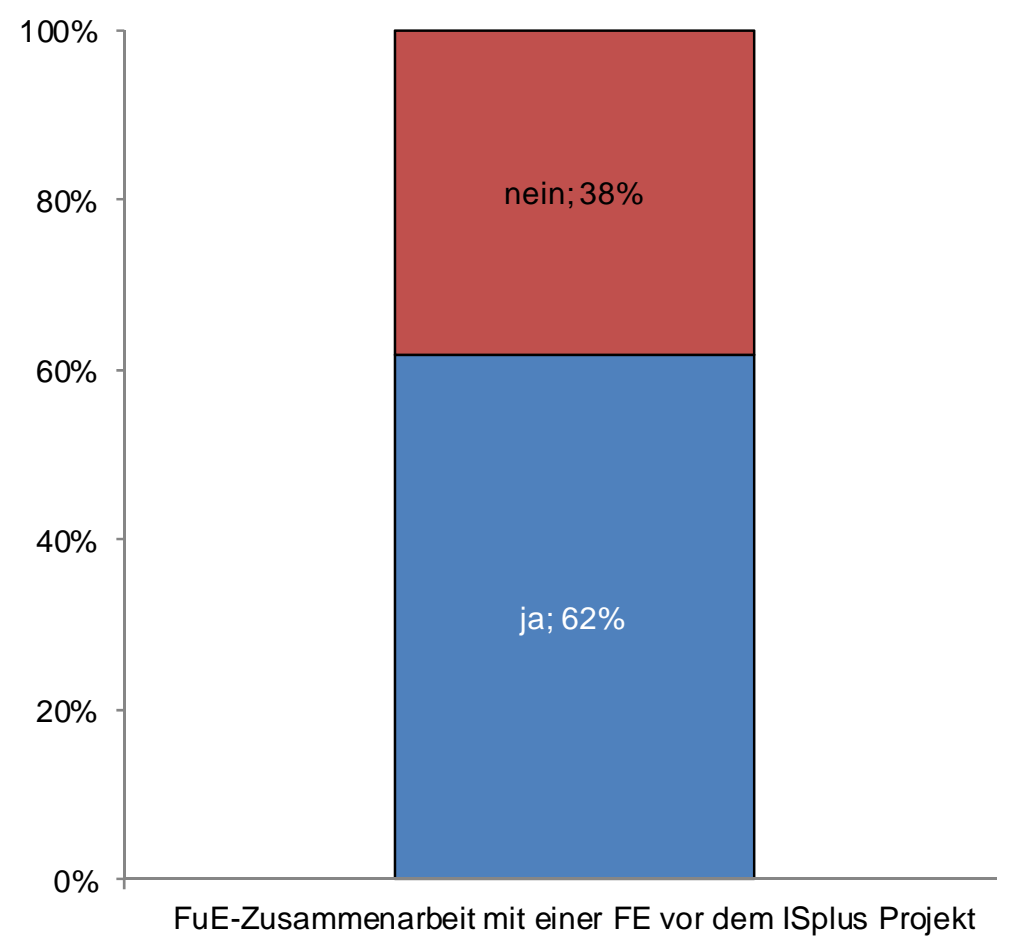

Quelle: Unternehmensbefragung, $\mathrm{n}=42$ 


\section{Grafik 25 Wissenserwerb und Nutzung des neuen Wissen}

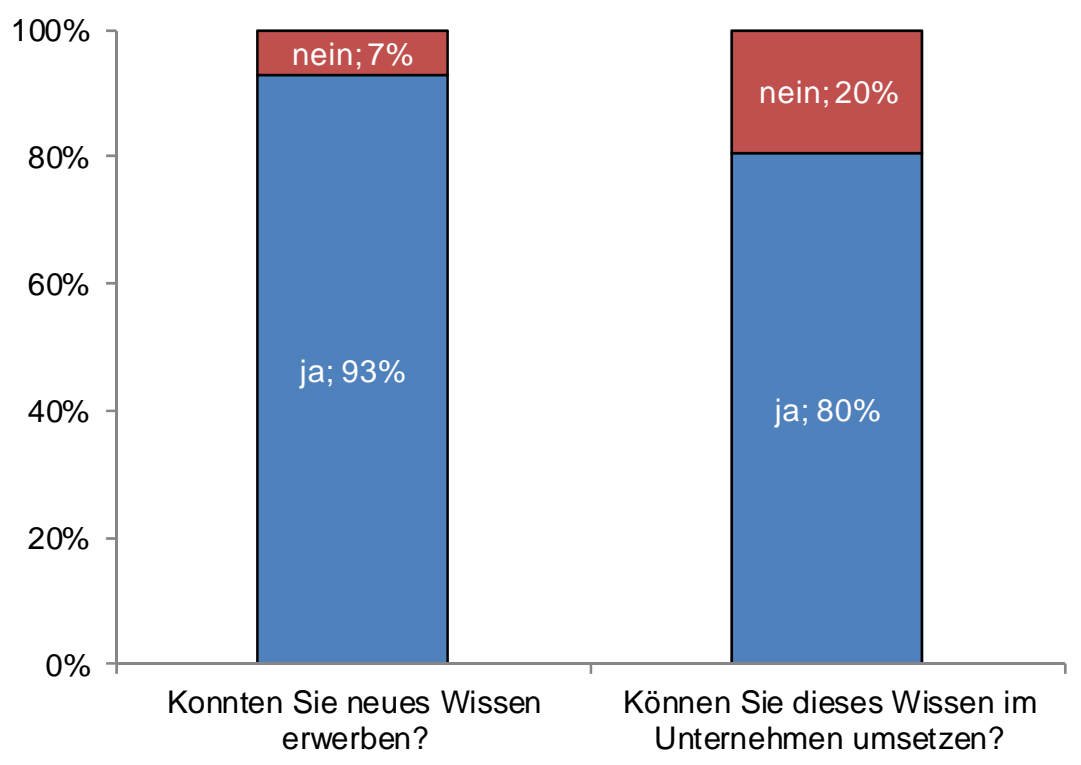

Quelle: Unternehmensbefragung, $n=42$

\section{Grafik 26 Zustandekommen der FEI Kooperation}

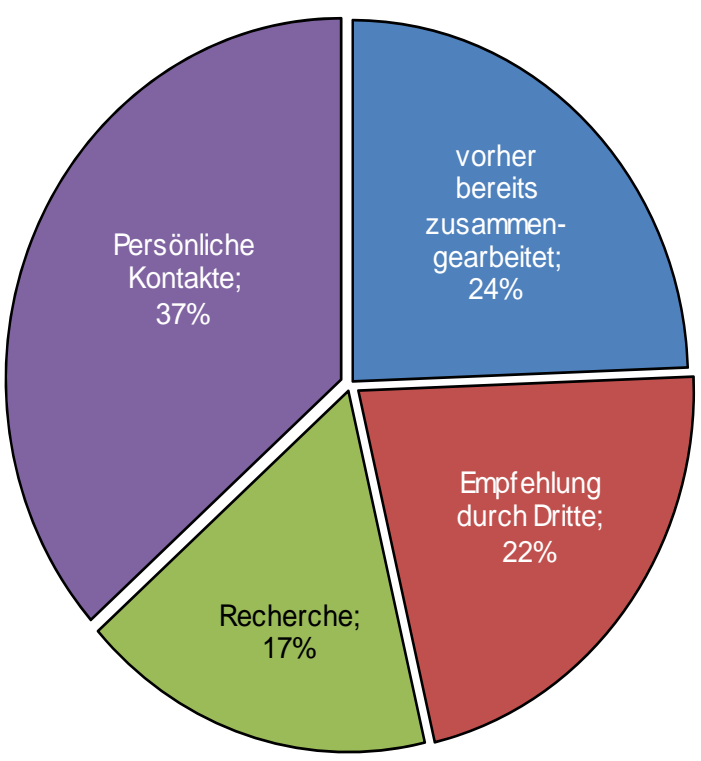

Quelle: Unternehmensbefragung, $n=42$ 


\section{Grafik 27 Unmittelbares Folgeprojekt}

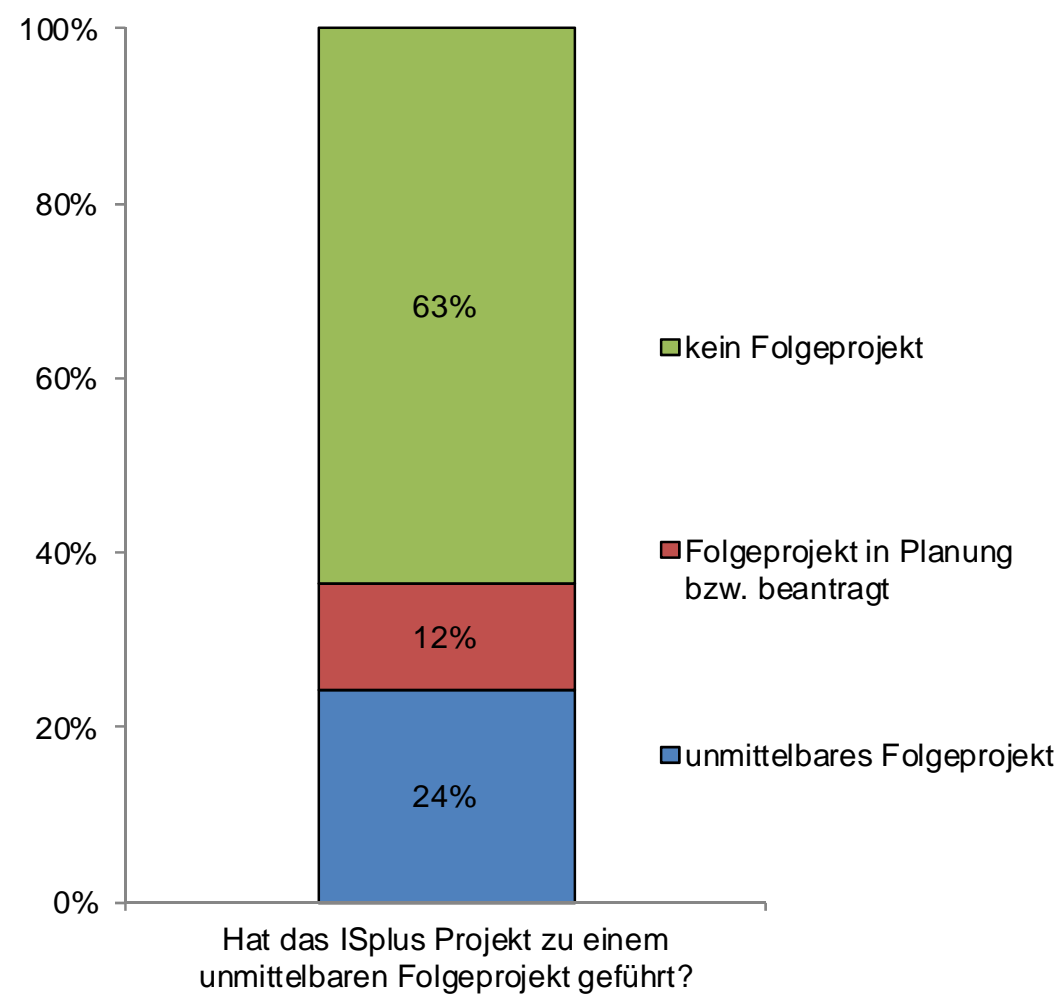

\section{Grafik $28 \quad$ Zufriedenheit mit FE}

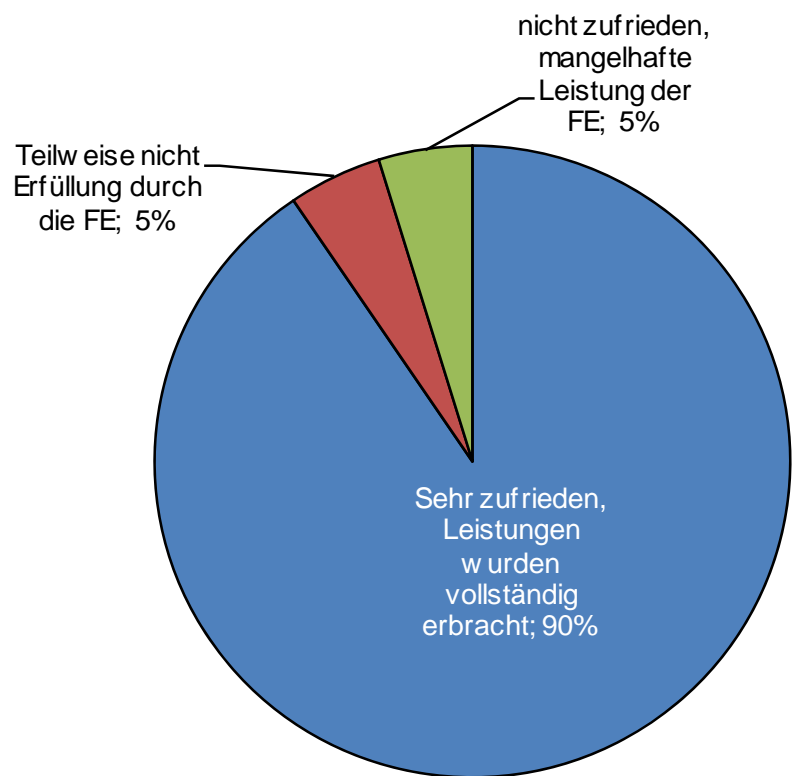

Quelle: Unternehmensbefragung, $n=42$; Die Frage nach der summarischen ZufrieQuelle: Unternehmensbefragung, $n=42$; Die Frage nach der summarischen Zufrie-
denheit über die Zusammenarbeit in einer Kooperation ist streng genommen kein evaluativer Tatbestand, sondern eine Zufriedenheitsanalyse. Diese soll aber in einem evaluativer Tatbestand, sondern eine Zufriedenheitsanalyse. Diese soll aber in einem
Kooperationsverhältnis, in dem das Unternehmen relativ stark von der Leistung der FE abhängt, auch seinen Platz haben.

Quelle: Unternehmensbefragung, $n=42$ 


\section{Grafik 29 Weitere Zusammenarbeit mit der Forschungseinheit}

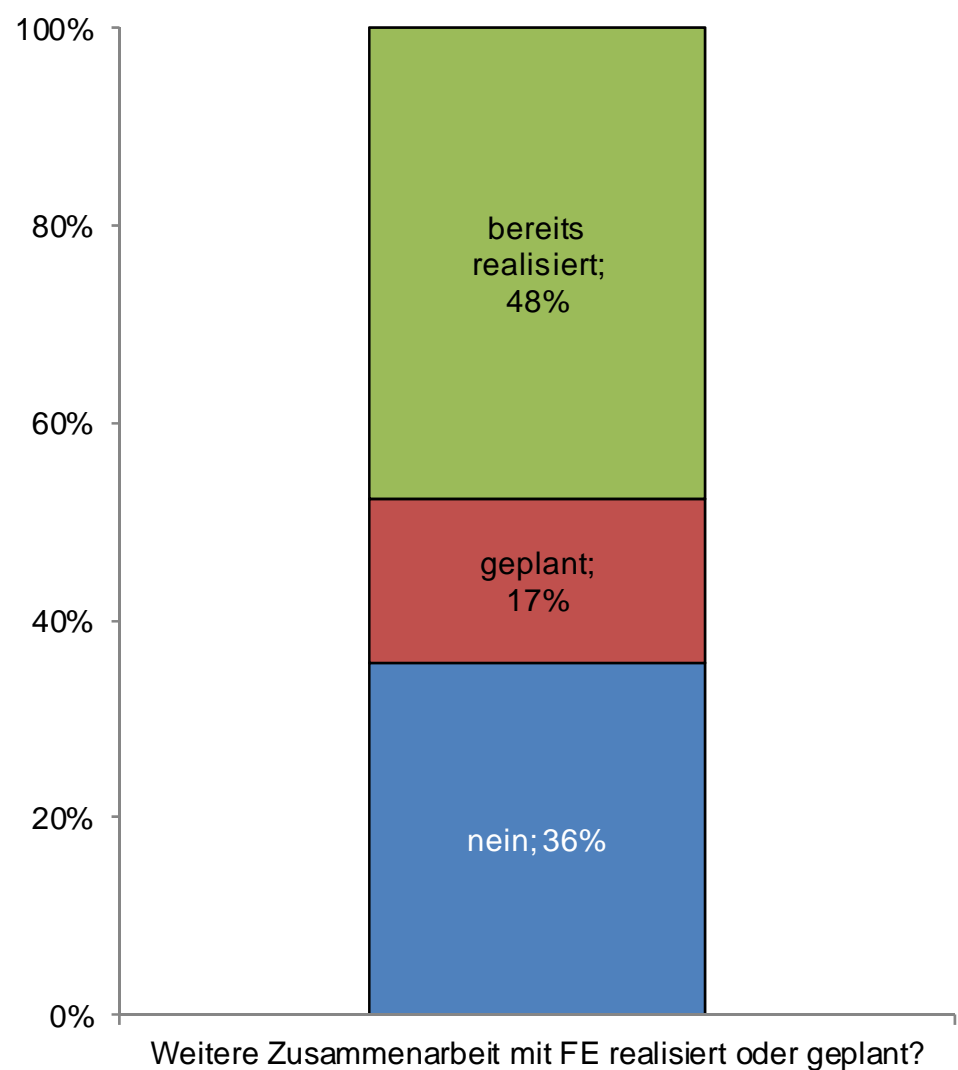

Quelle: Unternehmensbefragung, $n=42$

\section{Grafik $30 \quad$ Planung von Zusammenarbeit mit Forschungseinheiten}

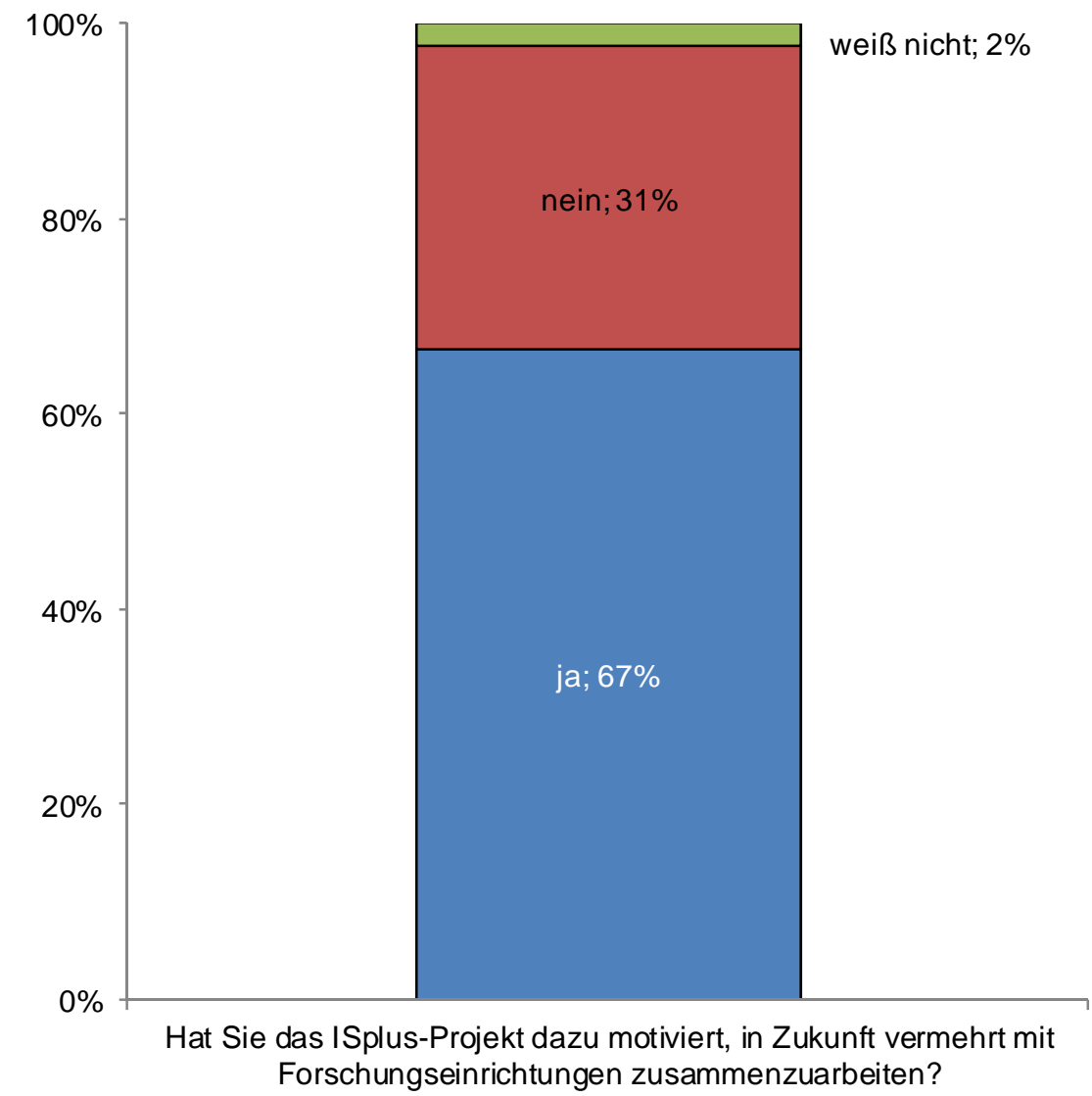

Quelle: Unternehmensbefragung, $\mathrm{n}=42$ 


\subsection{Eingesetzter Methodenmix}

Der Zeitraum für diese Evaluierung betrug knapp zwei Monate. Dies beeinflusste natürlich die Wahl des Methodenmix, der in der Folge dargestellt wird.

\section{Literatur- und Dokumentenanalyse}

Als Basis der Durchführung der Zwischenevaluierung des Programms Innovationsscheck Plus diente eine Analyse der vom Auftraggeber bzw. der FFG zur Verfügung gestellten Unterlagen und Dokumente (Strategiepapiere, Richtlinien und Programmdokumente der Innovationsschecks sowie der benachbarten Programme (FFG und aws), ein zufällig ausgewählter Auszug von 30 Projektendberichten des Innovationsschecks Plus, von je 30 Projekteendberichten des kleinen Innovationsschecks sowie der Feasibility Studien. Die Projektendberichte wurden anhand eines definierten Kriterienkatalogs einer systematischen Analyse unterzogen. Darüber hinaus gibt dieses Arbeitspaket eine Überblick bezüglich vorhandener Innovationsschecks (innovation voucher schemes) in anderen Ländern (UK, DE, IRL, CH, NL, CZ).

\section{Analyse der FFG Monitoringdaten}

Ausgangspunkt für die Arbeiten im Rahmen dieses Arbeitspakets bildete eine Analyse der von der FFG zur Verfügung gestellten Monitoringdaten per 17.10.2014. Diese beinhalten programmübergreifende Beteiligungen am FFG Portfolio, sowie Informationen zu den beiden Programmen Innovationsscheck und Innovationsscheck Plus. Darüber hinaus wurden seitens der FFG die Monatsblätter und Jahresberichte zum ISplus zur Verfügung gestellt, die ebenfalls in die Evaluierung einbezogen wurden. Die Analyse und Verarbeitung der übermittelten Daten ermöglichte nicht nur einen deskriptiven Überblick der geförderten Unternehmen und Forschungseinheiten, sondern gab auch Aufschluss bezüglich der Verknüpfung mit anderen Programmen bzw. dem Eintreten intendierter, programmbezogenen Wirkungen. Der Abgrenzungszeitraum der Evaluierung wurde per 30.6.2014 gewählt, damit drei volle Jahre des Programms ISplus analysiert werden konnten. In den Monitoringdaten wurde die Klassifikation von FE und Unternehmenspartnern einer Plausibilitätskontrolle unterzogen und bei Bedarf berichtigt. Die Monitoringdaten stellten die Grundlage für die Schichtung der repräsentativen Unternehmensbefragung dar.

Da die bisherige Laufzeit des ISplus-Programms und der Zeitpunkt der Evaluierung durchaus mit jenem des IS-Programms und dem Zeitpunkt der Evaluierung durch technopolis vergleichbar waren, konnten auch fallweise Vergleiche der empirischen Befunde angestellt werden. 


\section{Telefoninterviews mit Unternehmen}

Zur Befragung der begünstigten Unternehmen wurden telefonische Interviews durchgeführt. Die potenziellen Interviewpartner wurden dafür entlang relevanter Schichtungskriterien gruppiert (Unternehmensgröße, Art des Forschungspartner, vorgelagerter IS, FFG Erfahrung) und dann mit Hilfe der FFGMonitoringdaten zufällig ausgewählt. Insgesamt wurden 42 Telefoninterviews entlang eines teilweise standardisierten, und teilweise offenen Interviewleitfadens durchgeführt, die einen Innovationsscheck in Anspruch genommen haben und das betreffende Projekt vor dem 30.6.2014 abschließen konnten. Die Interviews mit den Unternehmen dauerten zwischen 20 und 60 Minuten und erstreckten sich über einen Zeitraum von rd. drei Wochen. Der Gesprächsinhalt wurde schriftlich festgehalten und für weiterführende Auswertungen elektronisch erfasst.

\section{Interviews mit Forschungseinheiten (10)}

Analog zu der Unternehmensbefragung wurden auch Vertreter von zehn Forschungseinrichtungen telefonisch bzw. persönlich interviewt. Die Auswahl der FE erfolgte primär nach ihrer Erfahrung im Umgang mit ISplus Projekten sowie nach ihrem institutionellen Hintergrund. Dieser Selektion folgend war es dennoch möglich, Forschungseinrichtungen unterschiedlichster thematischer Forschungsgebiete hinzuzuziehen, um ein breiteres Verständnis unterschiedlicher Wirkungsweisen erfassen zu können. Insgesamt fanden zehn Gespräche mit FE statt, wobei fünf mit außeruniversitären und fünf mit universitären Einrichtungen geführt wurden. Ebenfalls aufbauend auf einen Interviewleitfaden wurden die Fragestellungen gegenüber der Unternehmensbefragung offener gestaltet.

\section{Fokusgruppe mit Forschungseinheiten (6)}

Die Ergebnisse aus den Interviews mit den KMU und den Forschungseinheiten wurden im Vorfeld der Durchführung der Fokusgruppe ausgewertet und zu ersten Aussagen verdichtet. Diese boten in weiterer Folge die Diskussionsgrundlage für die Fokusgruppe und ergänzten die Abbildung einer Projektsicht aus der Sichtweise der Forschungspartner. Darüber hinaus wurden die Erfahrungen und die Expertise der FE auch in Hinblick auf die Relevanz und Effektivität komplementärer bzw. anschließender Förderangebote in Anspruch genommen. Die teilnehmenden FE stammten dabei aus dem außeruniversitären Forschungsbereich sowie den Fachhochschulen. Die Diskussion wurde mit Tonband aufgenommen. Die Auswertung erfolgte auf Basis eines Transkripts, anhand dessen sich relevante Kategorien der interessierenden Fragestellung bilden, und damit themenzentrierte Auswertungen erstellen lassen. 


\section{Reflexionsworkshop mit relevanten Stakeholdern}

Der Reflexionsworkshop mit relevanten Stakeholdern diente der ersten Präsentation und Diskussion vorläufiger Ergebnisse. Dieser wurde von der KMU Forschung Austria organisiert und relevante Stakeholder (Auftraggeber, den Projektverantwortlichen im bmwfw und in der FFG) eingeladen. Ziel des Workshops war die Generierung von Lerneffekten und die Konkretisierung von Empfehlungen zur Weiterentwicklung des Programms Innovationsscheck Plus. Die Ergebnisse der Diskussion wurden dann von der KMU Forschung Austria in den Endbericht eingearbeitet.

\subsection{Literatur}

Bakhshi, H., Edwards, J., Roper, St., Scully, J. Shaw, D., Morley L. and N. Rathbone (2013). Creative Credits: A Randomized Controlled Industrial Policy Experiment. funded by Nesta, ESRC, AHRC and Manchester City Council

Bayerisches Staatsministerium für Wirtschaft, Infrastruktur, Verkehr und Technologie (2011). Innovationsgutscheine für kleine Unternehmen/ Handwerksbetriebe, Projektbeispiele 2009 - 2011

BMWFW und BMVIT (2014). Österreichischer Forschungs- und Technologiebericht. Erstellt durch ein Konsortium, koordiniert durch Joanneum Research, Wien

Cornet, M., B. Vroomen and M. van der Steeg (2006). Do Innovation Vouchers Help SMEs to Cross the Bridge towards Science? CBP Discussion Paper No. 58. Netherlands Bureau for Economic Policy Analysis, The Hague

Department for Business Innovation \& Skills, 2012, 2013, sowie 2014. Annual Innovation Report: Innovation, Research and Growth. London

Enterprise Ireland (2010). Innovation Vouchers success stories. Funded by the Irish Government under the National Development Plan, 2007-2013

European Commission (2011). Implementation of a Transeuropean Knowledge Voucher System. KVoucher, Complementary Materials for Final Report Jund 2009 - May 2011“, Brussels

Flanagan, K., Uyarra, E. and M. Laranja (2011). Reconceptualising the 'policy mix' for innovation. Research Policy 40 (5): 702-713

Good, B. und B. Tiefenthaler (2011). Zwischenevaluierung des Programms Innovationsscheck, Endbericht. Technopolis Group, Wien

Hemert, P., Nijkamp, P. and E. Masurel (2013). From innovation to commercialization through networks and agglomerations: analysis of sources of inno- 
vation, innovation capabilities and performance of Dutch SMEs. The Annals of Regional Science, 50 (2): 425-452

IfM (2011). Begleitforschung zum Modellvorhaben Innovationsgutscheine für kleine und mittlere Unternehmen. Studie des Instituts für Mittelstandsforschung in Kooperation mit dem ZEW Mannheim, im Auftrag des Wirtschaftsministeriums Baden-Württemberg

IfS (2012). Evaluation der Fördermaßnahme "Gewährung von Innovationsprämien für kleine und mittlere Unternehmen im Freistaat Sachsen" (InnoPrämie). Studie des Instituts für Stadtforschung und Strukturpolitik GmbH im Auftrag des Sächsischen Staatsministeriums für Wissenschaft und Kunst

Matatkova, K. and J. Stejskal (2012). The Effectiveness of Public Support in the Form of Innovation Vouchers - Czech regional case. Recent Researches In Business and Economics, 83-88, ISBN: 978-1-61804-102-9

McLaughlin, J.A. and G.B. Jordan (1999). Logic Models: A Tool for Telling Your Program's Performance Story. Evaluation and Program Planning, Vol. 22 (1): 65-72

OECD Innovation Policy Platform (2010). Innovation Vouchers. OECD, Paris.

SF Council (2010). Evaluation of the first phase of the Scottish Funding Council Innovation Voucher Scheme. Edinburgh

Sonderrichtlinie Innovationsscheck $€$ 5.000,- des Bundesministeriums für Verkehr, Innovation und Technologie und des Bundesministeriums für Wirtschaft, Familie und Jugend, Fassung vom 1.1.2014, GZ BMVIT-610040/0003-III//2/2013

Sonderrichtlinie Innovationsscheck Plus des Bundesministeriums für Wirtschaft, Familie und Jugend, Fassung vom 1.1.2014, GZ BMWFJ-98.181/0027C1/11/2013

Statistik Austria (2014). Innovation, Ergebnisse der Innovationserhebung CIS 2012. Herausgegeben von der Statistik Austria, Wien

Stejskal, J. and K. Matatkova (2012). Innovation Vouchers as a Suitable Instrument for Effective Public Support of Innovations by Local Public Administration. International Journal of Systems Applications, Engineering \& Development, Vol. 6 (5): 333-341 
KMU Forschung Austria

Austrian Institute for SME Research 